\title{
Silylium-Ion Regeneration by Protodesilylation Enables Friedel-Crafts Alkylation with Less Isomerization and No Defunctionalization
}

\author{
Tao He, Hendrik F. T. Klare, ${ }^{\star}$ and Martin Oestreich* \\ Institut für Chemie, Technische Universität Berlin \\ Straße des 17. Juni 115, 10623 Berlin, Germany \\ martin.oestreich@tu-berlin.de
}

Supporting Information 


\section{Table of Contents}

1 General Information

S3

2 Experimental Details for the Synthesis of Silylated Arenes $6 a-6 d$ as well as (3-Fluoropropyl)benzene (1a)

2.1 General Procedure for the Synthesis of Silylated Arenes (GP 1)

S4

2.2 Characterization Data of Silylated Arenes 6a-6e

S4

2.3 Experimental Details for the Synthesis of (2,6-Dichlorophenyl)triethylsilane S6 (6e)

2.4 Experimental Details for the Synthesis of (3-Fluoropropyl)benzene (1a)

3 Experimental Details for the Silylium-Ion-Promoted Friedel-Crafts Alkylation

3.1 General Procedure for the Silylium-Ion-Promoted Friedel-Crafts Alkylation of Benzene with Addditive 6a (GP 2)

3.2 General Procedure for the Silylium-Ion-Promoted Friedel-Crafts Alkylation of Benzene without Additive (GP 3)

3.3 General Procedure for the Silylium-Ion-Promoted Friedel-Crafts Alkylation in 1,2-Dichlorobenzene (GP 4)

3.4 Crude ${ }^{1} \mathrm{H}$ NMR Spectra of the Silylium-lon-Promoted Friedel-Crafts Alkylation of Benzene with (3-Bromopropyl)benzene (1c): Assignment of the Products

3.5 Characterization Data of the Friedel-Crafts Products

4 Experimental Details of the Mechanistic Control Experiments

4.1 Protodesilylation of Triethyl(phenyl)silane (6a) with Reed's Benzenium Carborate $\left[\mathrm{C}_{6} \mathrm{H}_{7}\right]^{+}\left[\mathrm{HCB}_{11} \mathrm{H}_{5} \mathrm{Br}_{6}\right]^{-}$

4.2 Investigation of the Defunctionalization 


\section{General Information}

All reactions were performed in flame-dried glassware using an MBraun glovebox or conventional Schlenk techniques under a static pressure of argon (glovebox) or nitrogen (fume hood) unless otherwise stated. Solvent for chromatography and extraction were distilled prior to use. Tetrahydrofurane (THF) was dried voer sodium and freshly distilled prior to use. Toluene $\left(\mathrm{C}_{7} \mathrm{H}_{8}\right)$ was dried over calcium hydride, degassed by three freeze-pump-thaw cycles, and stored in a glovebox over thermally activated $4 \AA$ molecular sieves. Dry benzene $\left(\mathrm{C}_{6} \mathrm{H}_{6}\right)$ was obtained from an MBraun solvent purification system (SPS-800), degassed by three freeze-pump-thaw cycles, and stored in a glovebox over thermally activated $4 \AA$ molecular sieves. Trityl salt $\left[\mathrm{Ph}_{3} \mathrm{C}\right]^{+}\left[\mathrm{HCB}_{11} \mathrm{H}_{5} \mathrm{Br}_{6}\right]^{-,},{ }^{1}$ Reed's arenium carborate $\left[\mathrm{C}_{6} \mathrm{H}_{7}\right]^{+}\left[\mathrm{HCB}_{11} \mathrm{H}_{5} \mathrm{Br}_{6}\right]^{-,}{ }^{1,2}$ counteranion-stablized silylium ions $\left[\mathrm{Et}_{3} \mathrm{Si}\left(\mathrm{HCB}_{11} \mathrm{H}_{5} \mathrm{Br}_{6}\right)\right]^{1,3-5}$ and $\left[\mathrm{Me}_{3} \mathrm{Si}\left(\mathrm{HCB}_{11} \mathrm{H}_{5} \mathrm{Br}_{6}\right)\right]^{6}$ were synthesized according to reported procedures. Unless noted otherwise, all alkyl halides 1 (except 1a) and arenes 2 were purchased from commercial suppliers, degassed by three freeze-pump-thaw cycles, and stored in a glovebox over thermally activated $4 \AA$ molecular sieves. Analytical thin-layer chromatography (TLC) was performed on silica gel 60 F254 glass plates. Flash column chromatography was performed on silica gel $60(40-63 \mu \mathrm{m}, 230-400$ mesh ASTM) by VWR Chemicals using the indicated solvents. ${ }^{1} \mathrm{H},{ }^{13} \mathrm{C},{ }^{19} \mathrm{~F}$, and ${ }^{29} \mathrm{Si} \mathrm{NMR}$ spectra were recorded in $\mathrm{C}_{6} \mathrm{D}_{6}$ or $\mathrm{CDCl}_{3}$ on Bruker AV500 and Bruker AV400 instruments. $\mathrm{C}_{6} \mathrm{D}_{6}$ was degassed by three freeze-pump-thaw cycles and stored in a glovebox over thermally activated $4 \AA$ molecular sieves. Chemical shifts are reported in parts per million (ppm) and are referenced to the residual solvent resonance as the internal standard $\left(\mathrm{C}_{6} \mathrm{D}_{5} \mathrm{H}: \delta=7.16 \mathrm{ppm}\right.$ for ${ }^{1} \mathrm{H}$ NMR and $\mathrm{C}_{6} \mathrm{D}_{6}: \delta=128.06$ ppm for ${ }^{13} \mathrm{C} N M R ; \mathrm{CHCl}_{3}: \delta=7.26$ ppm for ${ }^{1} \mathrm{H}$ NMR and $\mathrm{CDCl}_{3}: \delta=77.16 \mathrm{ppm}$ for $\left.{ }^{13} \mathrm{C} \mathrm{NMR}\right) .{ }^{19} \mathrm{~F}$ and ${ }^{29} \mathrm{Si} \mathrm{NMR}$ spectra are referenced in compliance with the unified scale for NMR chemical shifts as recommended by the IUPAC stating the chemical shift relative to $\mathrm{CCl}_{3} \mathrm{~F}$ and TMS, respectively. ${ }^{7}$ Data are reported as follows: chemical shift, multiplicity ( $\mathrm{s}=$ singlet, $\mathrm{d}=$ doublet, $\mathrm{t}=$ triplet, $\mathrm{q}=$ quartet, $\mathrm{m}=$ multiplet, $\mathrm{br}=$ broad signal), coupling constants $(\mathrm{Hz})$, and integration. High resolution mass spectra (HRMS) were obtained from the Laboratory of Mass Spectrometry at the Institut für Chemie, Technische Universität Berlin. 


\section{Experimental Details for the Synthesis of Silylated Arenes 6a-6d as well as (3-Fluoropropyl)benzene (1a)}

\subsection{General Procedure for the Synthesis of Silylated Arenes (GP 1)}

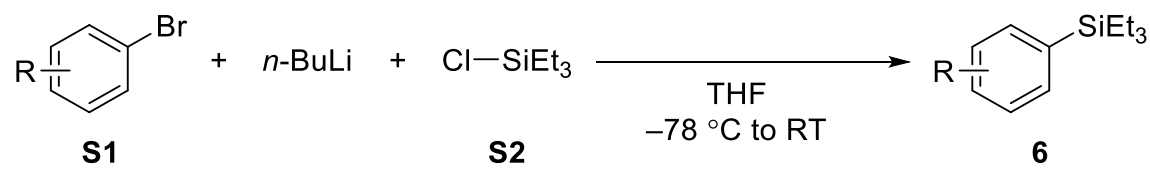

According to a reported procedure, ${ }^{8} n$-BuLi $(8.8 \mathrm{~mL}$ of a $2.5 \mathrm{M}$ solution in $n$-hexane, $22.0 \mathrm{mmol}$, 1.1 equiv) is added dropwise to a solution of the corresponding aryl bromide $\mathbf{S} 1(20.0 \mathrm{mmol}$, 1.0 equiv) in anhydrous THF $(30 \mathrm{~mL})$ at $-78^{\circ} \mathrm{C}$. The mixture is stirred at this temperature for additional $30 \mathrm{~min}$, followed by the addition of chlorotriethylsilane (S2, 3.3 g, $22.0 \mathrm{mmol}, 1.1$ equiv). The mixture is allowed to warmed to room temperature and stirred overnight. The reaction mixture is quenched by the addition of water $(15 \mathrm{~mL})$ and saturated aqueous $\mathrm{NH}_{4} \mathrm{Cl}$ solution $(25 \mathrm{~mL})$. The organic phase is separated, and the aqueous layer is extracted with EtOAc $(3 \times 20 \mathrm{~mL})$. The combined organic phases are dried over $\mathrm{MgSO}_{4}$ and concerated under reduced pressure. The residue is purified by flash column chromatography on silica gel using cyclohexane as eluent to afford the corresponding silylated arene 6 in analytically pure form.

\subsection{Characterization Data of Silylated Arenes 6a-6e}

\subsubsection{Triethyl(phenyl)silane (6a)}

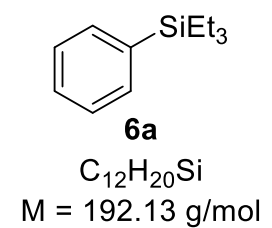

Prepared from bromobenzene $(3.2 \mathrm{~g}, 20.0 \mathrm{mmol})$ according to GP 1. Flash column chromatography on silica gel using cyclohexane afforded triethyl(phenyl)silane (6a) as a colorless oil (3.6 g, 95\% yield). ${ }^{1} \mathrm{H}$ NMR $\left(500 \mathrm{MHz}, \mathrm{CDCl}_{3}, 298 \mathrm{~K}\right): \delta=7.52-7.47(\mathrm{~m}, 2 \mathrm{H})$, 7.37-7.33 (m, 3H), $0.97(\mathrm{t}, J=8.0 \mathrm{~Hz}, 9 \mathrm{H}), 0.80(\mathrm{q}, J=7.9 \mathrm{~Hz}, 6 \mathrm{H}) \mathrm{ppm} .{ }^{13} \mathrm{C}\left\{{ }^{1} \mathrm{H}\right\}$ NMR $(126$ $\left.\mathrm{MHz}, \mathrm{CDCl}_{3}, 298 \mathrm{~K}\right): \delta=137.6,134.3,128.8,127.8,7.5,3.5 \mathrm{ppm} .{ }^{1} \mathrm{H} /{ }^{29} \mathrm{Si} \mathrm{HMQC} \mathrm{NMR}(500 / 99$ $\mathrm{MHz}, \mathrm{CDCl}_{3}, 298 \mathrm{~K}$, optimized for $J=7 \mathrm{~Hz}$ ): $\delta=0.97 / 1.5,0.80 / 1.5 \mathrm{ppm}$. The NMR spectroscopic data are in accordance with those reported. ${ }^{9}$

2.2.2 Triethyl $(p$-tolyl)silane $(\mathbf{6 b})$



$\mathrm{C}_{13} \mathrm{H}_{22} \mathrm{Si}$

$\mathrm{M}=206.15 \mathrm{~g} / \mathrm{mol}$ 
Prepared from 4-bromotoluene $(3.4 \mathrm{~g}, 20.0 \mathrm{mmol})$ according to $\mathrm{GP}$ 1. Flash column chromatography on silica gel using cyclohexane afforded triethyl(p-tolyl)silane (6b) as a colorless oil (4.0 g, 97\% yield). ${ }^{1} \mathrm{H}$ NMR (500 MHz, $\left.\mathrm{CDCl}_{3}, 298 \mathrm{~K}\right): \delta=7.42-7.37$ (m, 2H), 7.20-7.16 (m, 2H), $2.35(\mathrm{~s}, 3 \mathrm{H}), 0.97(\mathrm{t}, J=8.0 \mathrm{~Hz}, 9 \mathrm{H}), 0.78-0.75(\mathrm{~m}, 6 \mathrm{H}) \mathrm{ppm} .{ }^{13} \mathrm{C}\left\{{ }^{1} \mathrm{H}\right\}$ NMR $\left(126 \mathrm{MHz}, \mathrm{CDCl}_{3}, 298 \mathrm{~K}\right): \delta=138.6,134.4,133.8,128.7,21.6,7.6,3.6 \mathrm{ppm} .{ }^{1} \mathrm{H} /{ }^{29} \mathrm{Si} \mathrm{HMQC}$ NMR (500/99 MHz, $\mathrm{CDCl}_{3}, 298 \mathrm{~K}$, optimized for $\left.J=7 \mathrm{~Hz}\right): \delta=7.40 / 1.3,0.97 / 1.3,0.78 / 1.3$ ppm. The NMR spectroscopic data are in accordance with those reported. ${ }^{10}$

2.2.3 (3,5-Dimethylphenyl)triethylsilane (6c)

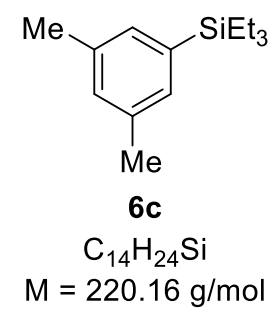

Prepared from 1-bromo-3,5-dimethylbenzene $(3.7 \mathrm{~g}, 20.0 \mathrm{mmol})$ according to GP 1. Flash column chromatography on silica gel using cyclohexane afforded (3,5-dimethylphenyl)triethylsilane (6c) as a colorless oil $\left(4.2 \mathrm{~g}, 95 \%\right.$ yield). ${ }^{1} \mathbf{H}$ NMR $\left(500 \mathrm{MHz}, \mathrm{CDCl}_{3}, 298 \mathrm{~K}\right): \delta=$ 7.12-7.09 (m, 2H), 7.02-6.98 (m, 1H), $2.33(\mathrm{~s}, 6 \mathrm{H}), 0.98(\mathrm{t}, J=8.1 \mathrm{~Hz}, 9 \mathrm{H}), 0.83-0.75(\mathrm{~m}, 6 \mathrm{H})$ ppm. ${ }^{13} \mathrm{C}\left\{{ }^{1} \mathrm{H}\right\}$ NMR $\left(126 \mathrm{MHz}, \mathrm{CDCl}_{3}, 298 \mathrm{~K}\right): \delta=137.4,137.0,132.0,130.6,21.6,7.6,3.6$ ppm. ${ }^{1} \mathrm{H} /{ }^{29} \mathrm{Si} \mathrm{HMQC} \mathrm{NMR} \mathrm{(500/99} \mathrm{MHz,} \mathrm{CDCl}_{3}, 298 \mathrm{~K}$, optimized for $\left.\mathrm{J}=7 \mathrm{~Hz}\right): \delta=7.10 / 1.2$, $0.98 / 1.2,0.78 / 1.2 \mathrm{ppm}$. The NMR spectroscopic data are in accordance with those reported. ${ }^{11}$ 2.2.4 (4-Chlorophenyl)triethylsilane (6d)

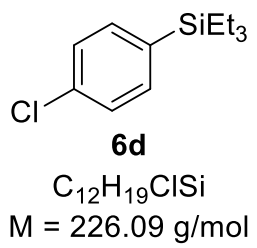

Prepared from 4-bromochlorobenzene $(3.8 \mathrm{~g}, 20.0 \mathrm{mmol}$ ) according to GP 1. Flash column chromatography on silica gel using cyclohexane afforded (4-chlorophenyl)triethylsilane (6d) as a colorless oil (3.7 g, 82\% yield). ${ }^{1} \mathrm{H}$ NMR $\left(500 \mathrm{MHz}, \mathrm{CDCl}_{3}, 298 \mathrm{~K}\right): \delta=7.43-7.37(\mathrm{~m}, 2 \mathrm{H})$, 7.34-7.31 (m, 2H), $0.95(\mathrm{t}, J=8.1 \mathrm{~Hz}, 9 \mathrm{H}), 0.81-0.75(\mathrm{~m}, 6 \mathrm{H})$ ppm. ${ }^{13} \mathrm{C}\left\{{ }^{1} \mathrm{H}\right\} \mathbf{N M R}(126 \mathrm{MHz}$, $\left.\mathrm{CDCl}_{3}, 298 \mathrm{~K}\right): \delta=135.8,135.7,135.2,128.1,7.4,3.4$ ppm. ${ }^{1} \mathrm{H} /{ }^{29} \mathrm{Si} \mathrm{HMQC} \mathrm{NMR}(500 / 99 \mathrm{MHz}$, $\mathrm{CDCl}_{3}, 298 \mathrm{~K}$, optimized for $J=7 \mathrm{~Hz}$ ): $\delta=7.40 / 2.0,0.95 / 2.0,0.78 / 2.0 \mathrm{ppm}$. The NMR spectroscopic data are in accordance with those reported. ${ }^{12}$ 


\subsection{Experimental Details for the Synthesis of (2,6-Dichlorophenyl)triethylsilane (6e)}

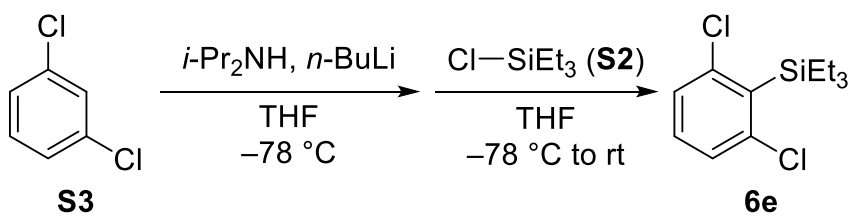

According to a reported procedure, ${ }^{13} \mathrm{n}$-BuLi $(4.4 \mathrm{~mL}$ of a $2.5 \mathrm{M}$ solution in $n$-hexane, 11.0 mmol, 1.1 equiv) was added dropwise to a solution of diisopropylamine (1.2 g, $12.0 \mathrm{mmol}, 1.2$ equiv) in anhydrous THF $(20 \mathrm{~mL})$ at $-78^{\circ} \mathrm{C}$. The mixture was stirred at this temperature for additional $30 \mathrm{~min}$. The freshly prepared LDA solution was then added dropwise to a solution of 1,3-dichlorobenzene (S3,1.5 g, $10.0 \mathrm{mmol}, 1.0$ equiv) at $-78{ }^{\circ} \mathrm{C}$. The mixture was stirred at this temperature for additional $1 \mathrm{~h}$, followed by the addition of chlorotriethylsilane $(\mathbf{S} 2,1.7 \mathrm{~g}$, $11.0 \mathrm{mmol}, 1.1$ equiv). The mixture was allowed to warmed to room temperature and stirred overnight. The reaction mixture was quenched by water $(10 \mathrm{~mL})$ and saturated aqueous $\mathrm{NH}_{4} \mathrm{Cl}$ solution $(20 \mathrm{~mL})$. The organic phase was separated, and the aqueous layer was extracted with EtOAc $(3 \times 20 \mathrm{~mL})$. The combined organic phases were dried over $\mathrm{MgSO}_{4}$ and concentrated under reduced pressure. The residue was purified by flash column chromatography on silica gel using cyclohexane as eluent to afford (2,6-dichlorophenyl)triethylsilane (6e) as a colorless oil in analytically pure form $(2.5 \mathrm{~g}, 96 \%$ yield $)$.

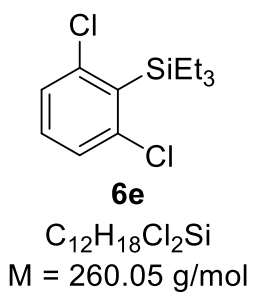

${ }^{1} \mathrm{H}$ NMR $\left(500 \mathrm{MHz}, \mathrm{CDCl}_{3}, 298 \mathrm{~K}\right): \delta=7.25-7.22(\mathrm{~m}, 2 \mathrm{H}), 7.18-7.13(\mathrm{~m}, 1 \mathrm{H}), 1.11-1.04(\mathrm{~m}$, $6 \mathrm{H}), 1.01-0.96(\mathrm{~m}, 9 \mathrm{H}) \mathrm{ppm} .{ }^{13} \mathrm{C}\left\{{ }^{1} \mathrm{H}\right\}$ NMR $\left(126 \mathrm{MHz}, \mathrm{CDCl}_{3}, 298 \mathrm{~K}\right): \delta=142.5,135.3,130.7$, 128.8, 8.0, 6.2 ppm. ${ }^{1} \mathrm{H} /{ }^{29} \mathrm{Si} \mathrm{HMQC} \mathrm{NMR}\left(500 / 99 \mathrm{MHz}, \mathrm{CDCl}_{3}, 298 \mathrm{~K}\right.$, optimized for $\left.\mathrm{J}=7 \mathrm{~Hz}\right)$ : $\delta=1.08 / 10.6,0.98 / 10.6 \mathrm{ppm}$. The NMR spectroscopic data are in accordance with those reported. ${ }^{14}$

\subsection{Experimental Details for the Synthsis of (3-Fluoropropyl)benzene (1a)}

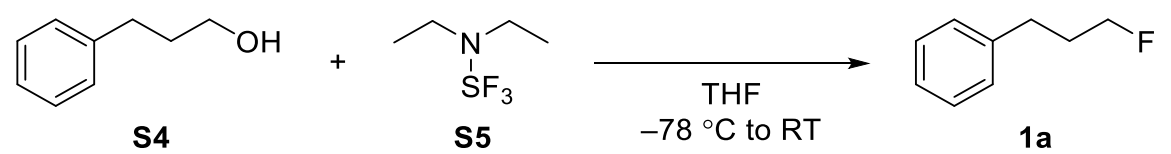

According to a reported procedure, ${ }^{15}$ to a solution of 3-phenyl-1-propanol (S4, $0.91 \mathrm{~g}, 6.7$ mmol, 1.0 equiv) in anhydrous THF (25 mL), (diethylamino)sulfur trifluoride (S5, $1.6 \mathrm{~g}, 10.0$ mmol, 1.5 equiv) was added at $-78^{\circ} \mathrm{C}$. The mixture was slowly warmed to room temperature and stirred for additional $2 \mathrm{~h}$. The reaction was then diluted with EtOAc $(20 \mathrm{~mL})$ and quenched with saturated aqueous $\mathrm{NaCl}$ solution $(20 \mathrm{~mL})$. The organic phase was separated, and the 
aqueous layer was extracted with EtOAc $(3 \times 20 \mathrm{~mL})$. The combined organic phases were dried over $\mathrm{MgSO}_{4}$ and concentrated under reduced pressure. The residue was purified by flash column chromatography on silica gel using cyclohexane as eluent to afford (3fluoropropyl)benzene (1a) as a colorless oil in analytically pure form (350 mg, 38\% yield).

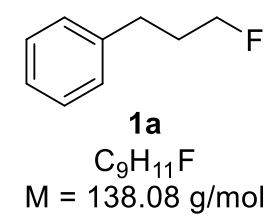

$\mathbf{R}_{\boldsymbol{f}}=0.20$ (cyclohexane). ${ }^{1} \mathrm{H}$ NMR $\left(500 \mathrm{MHz}, \mathrm{CDCl}_{3}, 298 \mathrm{~K}\right): \delta=7.33-7.28(\mathrm{~m}, 2 \mathrm{H}), 7.24-7.18$ $(\mathrm{m}, 3 \mathrm{H}), 4.46(\mathrm{dt}, J=47.2,6.0 \mathrm{~Hz}, 2 \mathrm{H}), 2.76(\mathrm{t}, J=7.6 \mathrm{~Hz}, 2 \mathrm{H}), 2.08-1.96(\mathrm{~m}, 2 \mathrm{H}) \mathrm{ppm} .{ }^{13} \mathrm{C}\left\{{ }^{1} \mathrm{H}\right\}$ NMR (126 MHz, $\mathrm{CDCl}_{3}, 298 \mathrm{~K}$ ): $\delta=141.3,128.6,128.6,126.2,83.3\left(\mathrm{~d}, \mathrm{~J}_{\mathrm{C}, \mathrm{F}}=164.8 \mathrm{~Hz}\right), 32.2$ $\left(\mathrm{d}, J_{\mathrm{C}, \mathrm{F}}=19.9 \mathrm{~Hz}\right), 31.4\left(\mathrm{~d}, \mathrm{~J}_{\mathrm{C}, \mathrm{F}}=4.9 \mathrm{~Hz}\right) \mathrm{ppm}$. The NMR spectroscopic data are in accordance with those reported. ${ }^{15}$ 
3 Experimental Details for the Silylium-Ion-Promoted Friedel-Crafts Alkylation

\subsection{General Procedure for the Silylium-Ion-Promoted Friedel-Crafts Alkylation of Benzene with Additive 6a (GP 2)}

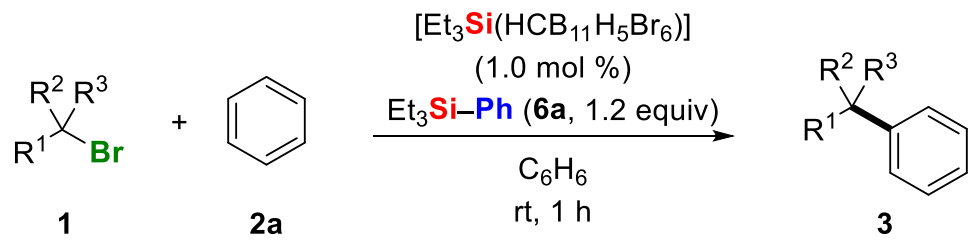

In a glovebox, silylium carborate $\left[\mathrm{Et}_{3} \mathrm{Si}\left(\mathrm{HCB}_{11} \mathrm{H}_{5} \mathrm{Br}_{6}\right)\right](2.9 \mathrm{mg}, 0.004 \mathrm{mmol}, 1.0 \mathrm{~mol} \%)$ is suspended in benzene $(0.2 \mathrm{~mL}$ ) and treated with triethyl(phenyl)silane (6a, $92.3 \mathrm{mg}, 1.2$ equiv). A solution of alkyl bromide $1(0.4 \mathrm{mmol}, 1.0$ equiv) in benzene $(0.8 \mathrm{~mL})$ is added dropwise, and the resulting mixture is stirred at ambient temperature for $1 \mathrm{~h}$. Cyclohexane $(22.0 \mu \mathrm{L}, 0.2$ mmol, 0.5 equiv) is added as an internal standard to determine the yield by ${ }^{1} \mathrm{H}$ NMR spectroscopy. Purification by flash column chromatography on silica gel using $n$-pentane as eluent affords the Friedel-Crafts alkylation product $\mathbf{3}$ in analytically pure form.

\subsection{General Procedure for the Silylium-Ion-Promoted Friedel-Crafts Alkylation of Benzene Without Additive (GP 3)}

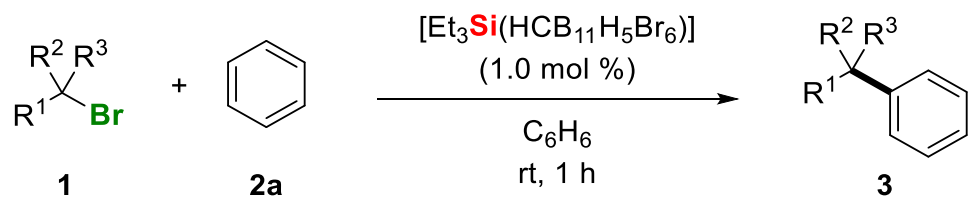

In a glovebox, silylium carborate $\left[\mathrm{Et}_{3} \mathrm{Si}\left(\mathrm{HCB}_{11} \mathrm{H}_{5} \mathrm{Br}_{6}\right)\right](1.5 \mathrm{mg}, 0.002 \mathrm{mmol}, 1.0 \mathrm{~mol} \%)$ is suspended in benzene $(0.1 \mathrm{~mL})$. A solution of the alkyl bromide 1 (0.2 mmol, 1.0 equiv) in benzene $(0.4 \mathrm{~mL})$ is added dropwise, and the resulting mixture is stirred at ambient temperature for $1 \mathrm{~h}$. Cyclohexane $(22.0 \mu \mathrm{L}, 0.2 \mathrm{mmol}, 1.0$ equiv) is added as an internal standard to determine the yield by ${ }^{1} \mathrm{H}$ NMR spectroscopy. Purification by flash column chromatography on silica gel using $n$-pentane as eluent affords the Friedel-Crafts alkylation product $\mathbf{3}$ in analytically pure form.

\subsection{General Procedure for the Silylium-Ion-Promoted Friedel-Crafts Alkylation in 1,2-} Dichlorobenzene (GP 4)

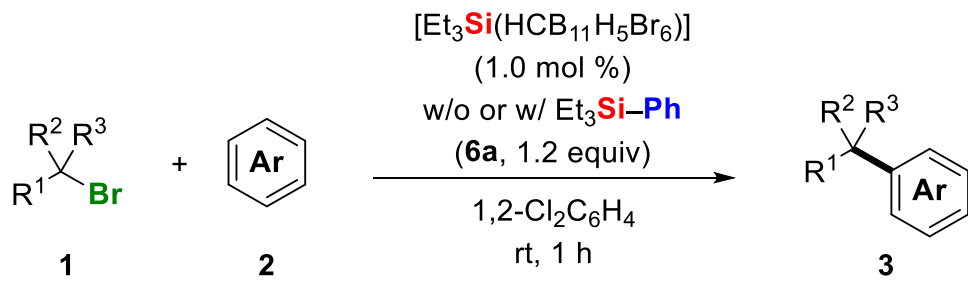


In a glovebox, silylium carborate $\left[\mathrm{Et}_{3} \mathrm{Si}\left(\mathrm{HCB}_{11} \mathrm{H}_{5} \mathrm{Br}_{6}\right)\right](2.9 \mathrm{mg}, 0.004 \mathrm{mmol}, 1.0 \mathrm{~mol} \%)$ is suspended in 1,2-dichlorobenzene $(0.2 \mathrm{~mL}$ ) and treated with (or without) triethyl(phenyl)silane (6a, $92.3 \mathrm{mg}, 1.2$ equiv). The corresponding arene 2 (2.0 mmol, 5.0 equiv) is added. A solution of the organobromide 1 ( $0.4 \mathrm{mmol}, 1.0$ equiv) in 1,2-dichlorobenzene $(0.8 \mathrm{~mL})$ is then added dropwise, and the resulting mixture is stirred at ambient temperature for $1 \mathrm{~h}$. Cyclohexane (22.0 $\mu \mathrm{L}, 0.2 \mathrm{mmol}, 0.5$ equiv) is added as an internal standard to determine the yield by ${ }^{1} \mathrm{H}$ NMR spectroscopy. Purification by flash column chromatography on silica gel using $n$-pentane as eluent affords the Friedel-Crafts alkylation product $\mathbf{3}$ in analytically pure form.

\subsection{Crude ${ }^{1} \mathrm{H}$ NMR Spectra of the Silylium-Ion-Promoted Friedel-Crafts Alkylation of} Benzene with (3-Bromopropyl)benzene (1c): Assignment of the Products

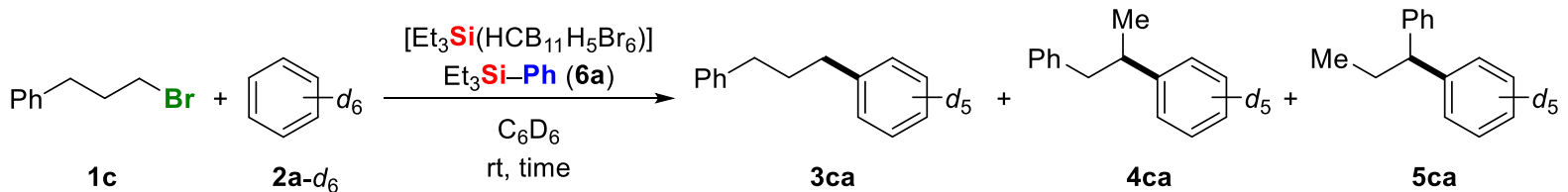

The reaction was performed according to GP 2 with (3-bromopropyl)benzene (1c, $39.8 \mathrm{mg}$, $0.20 \mathrm{mmol}, 1.0$ equiv) and triethyl(phenyl)silane (2a, $57.7 \mathrm{mg}, 1.5$ equiv) using silylium carborate [ $\left.\mathrm{Et}_{3} \mathrm{Si}\left(\mathrm{HCB}_{11} \mathrm{H}_{5} \mathrm{Br}_{6}\right)\right](1.5 \mathrm{mg}, 2.0 \mu \mathrm{mol}, 1.0 \mathrm{~mol} \%)$ in $\mathrm{C}_{6} \mathrm{D}_{6}(0.5 \mathrm{~mL})$. A sample of the reaction mixture was dissolved in $\mathrm{C}_{6} \mathrm{D}_{6}$ and directly subjected to NMR spectroscopy using cyclohexane ( $22.0 \mu \mathrm{L}, 0.20 \mathrm{mmol}, 1.0$ equiv) as an internal standard. Complete conversion of (3-bromopropyl)benzene (1c) was obsered. The formation of $3 \mathbf{c a}(71 \%)$ as the main product along with 4ca (14\%) and 5ca (14\%) was detected (Figure S1).

Selected NMR spectroscopic data for $3 \mathrm{ca}:{ }^{1} \mathrm{H}$ NMR $\left(500 \mathrm{MHz}, \mathrm{C}_{6} \mathrm{D}_{6}, 298 \mathrm{~K}\right): \delta=2.47$ (t, $\mathrm{J}=$ $7.7 \mathrm{~Hz}, 4 \mathrm{H}), 1.84-1.76(\mathrm{~m}, 2 \mathrm{H}) \mathrm{ppm}$. The NMR spectroscopic data are in accordance with those reported. ${ }^{16}$

Selected NMR spectroscopic data for 4ca: ${ }^{1} \mathbf{H}$ NMR $\left(500 \mathrm{MHz}, \mathrm{C}_{6} \mathrm{D}_{6}, 298 \mathrm{~K}\right)$ : $\delta=2.92-2.77$ $(\mathrm{m}, 2 \mathrm{H}), 2.65-2.59(\mathrm{~m} \mathrm{1H}), 1.13(\mathrm{~d}, J=6.8 \mathrm{~Hz}, 3 \mathrm{H}) \mathrm{ppm}$. The NMR spectroscopic data are in accordance with those reported. ${ }^{17}$

Selected NMR spectroscopic data for 5 ca: ${ }^{1} \mathbf{H}$ NMR $\left(500 \mathrm{MHz}, \mathrm{C}_{6} \mathrm{D}_{6}, 298 \mathrm{~K}\right): \delta=3.64(\mathrm{t}, \mathrm{J}=$ $7.8 \mathrm{~Hz}, 1 \mathrm{H}$ ), 1.93 (dq, $J=7.8 \mathrm{~Hz}, J=7.8 \mathrm{~Hz}, 2 \mathrm{H}$ ) ppm. The NMR spectroscopic data are in accordance with those reported. ${ }^{18}$ 


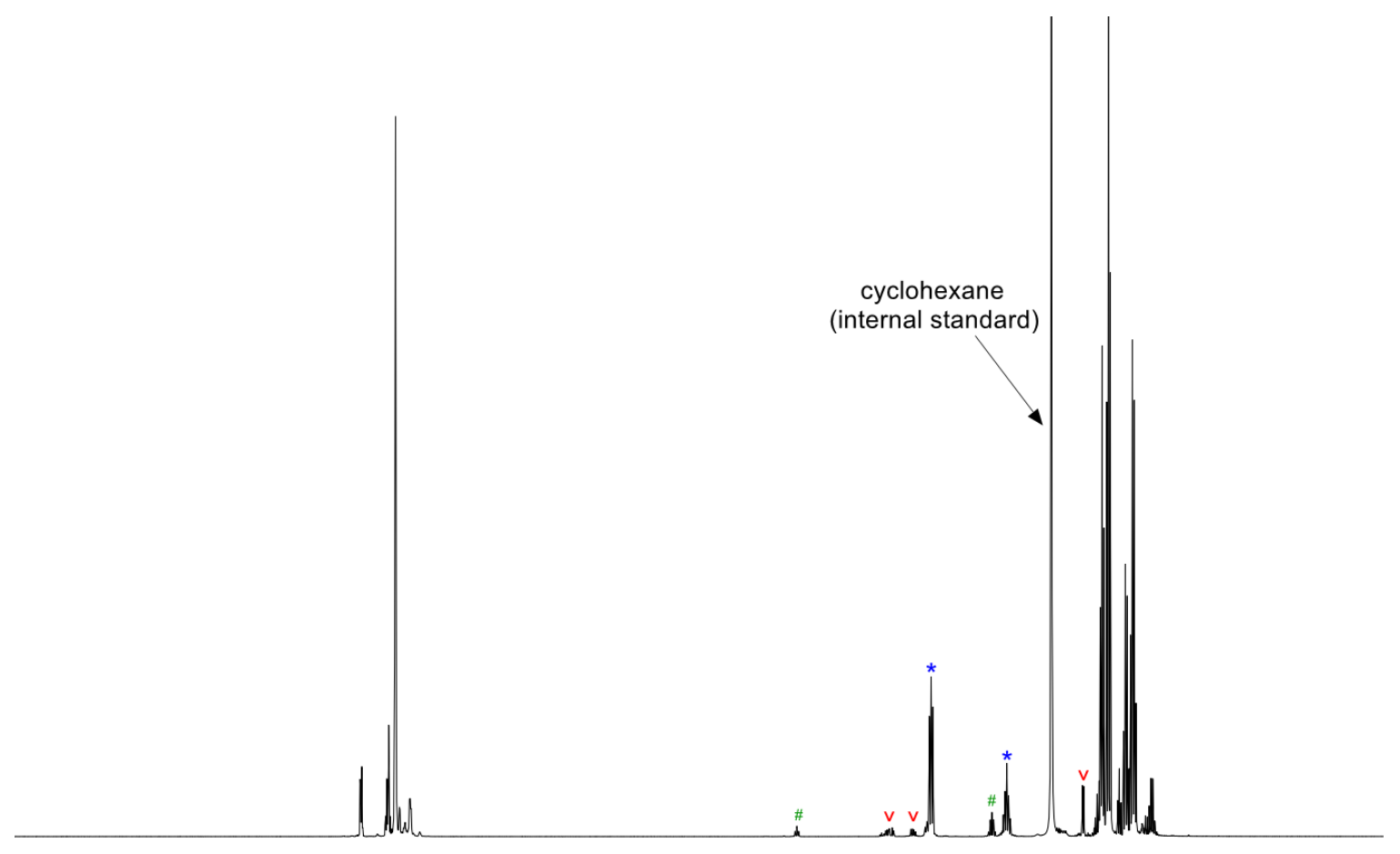

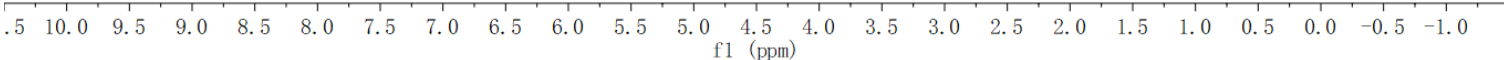



Figure S1. ${ }^{1} \mathrm{H}$ NMR spectra $\left(500 \mathrm{MHz}, \mathrm{C}_{6} \mathrm{D}_{6}, 298 \mathrm{~K}\right)$ of the the silylium-ion-promoted FriedelCrafts alkylation of benzene- $d_{6}\left(\mathbf{2 a}-d_{6}\right)$ with (3-bromopropyl)benzene (1c) (top) and enlarged section of the high field region (bottom). ( ${ }^{*}$ for $3 \mathrm{ca}, \mathrm{v}$ for $\mathbf{4 c a}$, and \# for $5 \mathrm{ca})$ 


\subsection{Characterization Data of the Friedel-Crafts Products}

\subsubsection{Diphenylmethane (3ea)}

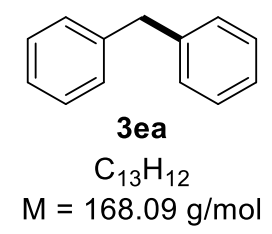

Prepared from (bromomethyl)benzene (1e, $68.4 \mathrm{mg}, 0.40 \mathrm{mmol}, 1.0$ equiv) with (or without) triethyl(phenyl)silane (6a, $92.3 \mathrm{mg}, 0.48 \mathrm{mmol}, 1.2$ equiv) according to GP 2 (or GP 3 ) (90\% NMR yield). Purification by flash column chromatography on silica gel using $n$-pentane afforded diphenylmethane (3ea) as a colorless oil (41.2 $\mathrm{mg}, 62 \%$ yield). $\mathbf{R}_{\boldsymbol{f}}=0.29$ (cyclohexane). ${ }^{1} \mathrm{H}$ NMR $\left(500 \mathrm{MHz}, \mathrm{CDCl}_{3}, 298 \mathrm{~K}\right): \delta=7.33-7.27(\mathrm{~m}, 4 \mathrm{H}), 7.25-7.18(\mathrm{~m}, 6 \mathrm{H})$, $4.01(\mathrm{~s}, 2 \mathrm{H}) \mathrm{ppm} .{ }^{13} \mathrm{C}\left\{{ }^{1} \mathrm{H}\right\}$ NMR $\left(126 \mathrm{MHz}, \mathrm{CDCl}_{3}, 298 \mathrm{~K}\right): \delta=141.3,129.1,128.6,126.2,42.1$ ppm. HRMS (APCl): calculated for $\mathrm{C}_{13} \mathrm{H}_{11}{ }^{+}[\mathrm{M}-\mathrm{H}]^{++}$: 167.0861; found 167.0852. The NMR spectroscopic data are in accordance with those reported. ${ }^{19}$

\subsubsection{1-Benzyl-4-methylbenzene (3fa)}

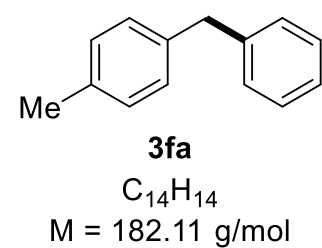

Prepared from 1-(bromomethyl)-4-methylbenzene (1f, $74.0 \mathrm{mg}, 0.40 \mathrm{mmol}, 1.0$ equiv) using triethyl(phenyl)silane (6a, $92.3 \mathrm{mg}, 0.48 \mathrm{mmol}, 1.2$ equiv) according to GP 2 ( $91 \%$ NMR yield). Purification by flash column chromatography on silica gel using $n$-pentane afforded 1-benzyl4-methylbenzene (3fa) as a colorless oil (51.7 mg, 71\% yield). $\mathbf{R}_{\boldsymbol{f}}=0.26$ (cyclohexane). ${ }^{1} \mathbf{H}$ $\operatorname{NMR}\left(500 \mathrm{MHz}, \mathrm{CDCl}_{3}, 298 \mathrm{~K}\right): \delta=7.34-7.27(\mathrm{~m}, 2 \mathrm{H}), 7.25-7.16(\mathrm{~m}, 3 \mathrm{H}), 7.14-7.10(\mathrm{~m}, 4 \mathrm{H})$, $3.98(\mathrm{~s}, 2 \mathrm{H}), 2.35(\mathrm{~s}, 3 \mathrm{H}) \mathrm{ppm} .{ }^{13} \mathrm{C}\left\{{ }^{1} \mathrm{H}\right\}$ NMR $\left(126 \mathrm{MHz}, \mathrm{CDCl}_{3}, 298 \mathrm{~K}\right): \delta=141.6,138.2,135.7$, 129.3, 129.0, 128.9, 128.6, 126.1, 41.7, 21.2 ppm. HRMS (APCI): calculated for $\mathrm{C}_{14} \mathrm{H}_{13^{*}}{ }^{*} \mathrm{M}-$ $\mathrm{H}]^{0+}:$ 181.1017; found 181.1009. The NMR spectroscopic data are in accordance with those reported. ${ }^{19}$

\subsubsection{1-Benzyl-3-methylbenzene (3ga)}



Prepared from 1-(bromomethyl)-3-methylbenzene $(\mathbf{1 g}, 74.0 \mathrm{mg}, 0.40 \mathrm{mmol}, 1.0$ equiv) using triethyl(phenyl)silane (6a, $92.3 \mathrm{mg}, 0.48 \mathrm{mmol}, 1.2$ equiv) according to GP 2 ( $81 \%$ NMR yield). Purification by flash column chromatography on silica gel using $n$-pentane afforded 1 -benzyl- 
3-methylbenzene (3ga) as a colorless oil (39.3 $\mathrm{mg}, 54 \%$ yield). $\mathbf{R}_{\boldsymbol{f}}=0.30$ (cyclohexane). ${ }^{1} \mathbf{H}$ NMR $\left(500 \mathrm{MHz}, \mathrm{CDCl}_{3}, 298 \mathrm{~K}\right): \delta=7.35-7.28(\mathrm{~m}, 2 \mathrm{H}), 7.25-7.18(\mathrm{~m}, 4 \mathrm{H}), 7.09-6.99(\mathrm{~m}, 3 \mathrm{H})$, $3.98(\mathrm{~s}, 2 \mathrm{H}), 2.34(\mathrm{~s}, 3 \mathrm{H}) \mathrm{ppm} .{ }^{13} \mathrm{C}\left\{{ }^{1} \mathrm{H}\right\} \mathrm{NMR}\left(126 \mathrm{MHz}, \mathrm{CDCl}_{3}, 298 \mathrm{~K}\right): \delta=141.4,141.2,138.2$, 129.9, 129.1, 128.6, 128.5, 127.0, 126.1, 126.1, 42.0, 21.6 ppm. HRMS (APCI): calculated for $\mathrm{C}_{14} \mathrm{H}_{13}{ }^{*}[\mathrm{M}-\mathrm{H}]^{+*}:$ 181.1017; found 181.1009. The NMR spectroscopic data are in accordance with those reported. ${ }^{20}$

\subsubsection{1-Benzyl-2-methylbenzene (3ha)}

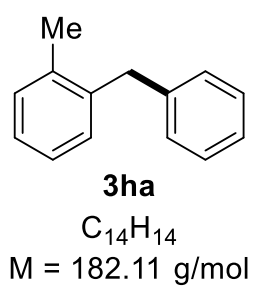

Prepared from 1-(bromomethyl)-2-methylbenzene (1h, $74.0 \mathrm{mg}, 0.40 \mathrm{mmol}, 1.0$ equiv) using triethyl(phenyl)silane (6a, $92.3 \mathrm{mg}, 0.48 \mathrm{mmol}, 1.2$ equiv) according to GP 2 (67\% NMR yield). Purification by flash column chromatography on silica gel using $n$-pentane afforded 1-benzyl2-methylbenzene (3ha) as a colorless oil ( $40.1 \mathrm{mg}, 55 \%$ yield). $\mathbf{R}_{\boldsymbol{f}}=0.32$ (cyclohexane). ${ }^{1} \mathbf{H}$ NMR $\left(500 \mathrm{MHz}, \mathrm{CDCl}_{3}, 298 \mathrm{~K}\right): \delta=7.33-7.26(\mathrm{~m}, 2 \mathrm{H}), 7.24-7.10(\mathrm{~m}, 7 \mathrm{H}), 4.01(\mathrm{~s}, 2 \mathrm{H}), 2.27$ (s, 3H) ppm. ${ }^{13} \mathrm{C}\left\{{ }^{1} \mathrm{H}\right\}$ NMR $\left(126 \mathrm{MHz}, \mathrm{CDCl}_{3}, 298 \mathrm{~K}\right): \delta=140.5,139.1,136.8,130.4,130.1$, 128.9, 128.5, 126.6, 126.1, 126.1, 39.6, 19.8 ppm. HRMS (APCI): calculated for $\mathrm{C}_{14} \mathrm{H}_{13^{+}}{ }^{*} \mathrm{M}-$ $H]^{\circ+}$ : 181.1017; found 181.1008. The NMR spectroscopic data are in accordance with those reported. ${ }^{21}$

\subsubsection{1-Benzyl-4-fluorobenzene (3ia)}



Prepared from 1-(bromomethyl)-4-fluorobenzene (1i, $75.6 \mathrm{mg}, 0.40 \mathrm{mmol}, 1.0$ equiv) using triethyl(phenyl)silane (6a, $92.3 \mathrm{mg}, 0.48 \mathrm{mmol}, 1.2$ equiv) according to GP 2 (90\% NMR yield). Purification by flash column chromatography on silica gel using $n$-pentane afforded 1-Benzyl4-fluorobenzene (3ia) as a colorless oil (52.0 mg, 70\% yield). $\mathbf{R}_{f}=0.30$ (cyclohexane). ${ }^{1} \mathbf{H}$ NMR $\left(500 \mathrm{MHz}, \mathrm{CDCl}_{3}, 298 \mathrm{~K}\right): \delta=7.36-7.28(\mathrm{~m}, 2 \mathrm{H}), 7.26-7.21(\mathrm{~m}, 1 \mathrm{H}), 7.21-7.13(\mathrm{~m}, 4 \mathrm{H}), 7.02-$ $6.94(\mathrm{~m}, 2 \mathrm{H}), 3.97(\mathrm{~s}, 2 \mathrm{H}) \mathrm{ppm} .{ }^{13} \mathrm{C}\left\{{ }^{1} \mathrm{H}\right\} \mathrm{NMR}\left(126 \mathrm{MHz}, \mathrm{CDCl}_{3}, 298 \mathrm{~K}\right): \delta=161.6\left(\mathrm{~d}, \mathrm{~J}_{\mathrm{C}, \mathrm{F}}=\right.$ $244.5 \mathrm{~Hz}$ ), 141.1, 136.9 (d, J $\left.J_{\mathrm{C}, \mathrm{F}}=2.4 \mathrm{~Hz}\right), 130.4\left(\mathrm{~d}, J_{\mathrm{C}, \mathrm{F}}=7.9 \mathrm{~Hz}\right), 129.0,128.7,126.2,115.3$ $\left(\mathrm{d}, J_{\mathrm{C}, \mathrm{F}}=21.2 \mathrm{~Hz}\right.$ ), $41.2 \mathrm{ppm}$. HRMS (APCl): calculated for $\mathrm{C}_{13} \mathrm{H}_{10} \mathrm{~F}^{\cdot+}[\mathrm{M}-\mathrm{H}]^{++}:$185.0767; found 185.0758. The NMR spectroscopic data are in accordance with those reported. ${ }^{19}$ 


\subsubsection{1-Benzyl-3-fluorobenzene (3ja)}

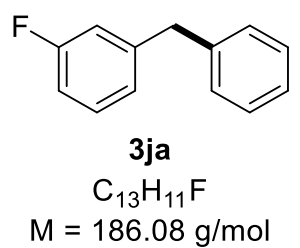

Prepared from 1-(bromomethyl)-3-fluorobenzene (1j, $75.6 \mathrm{mg}, 0.40 \mathrm{mmol}, 1.0$ equiv) using triethyl(phenyl)silane (6a, $92.3 \mathrm{mg}, 0.48 \mathrm{mmol}, 1.2$ equiv) according to GP 2 (>95\% NMR yield). Purification by flash column chromatography on silica gel using $n$-pentane afforded 1-benzyl3-fluorobenzene (3ja) as a colorless oil ( $60.9 \mathrm{mg}, 82 \%$ yield). $\mathbf{R}_{\boldsymbol{f}}=0.32$ (cyclohexane). ${ }^{1} \mathbf{H}$ NMR $\left(500 \mathrm{MHz}, \mathrm{CDCl}_{3}, 298 \mathrm{~K}\right): \delta=7.38-7.32(\mathrm{~m}, 2 \mathrm{H}), 7.32-7.20(\mathrm{~m}, 4 \mathrm{H}), 7.01(\mathrm{~d}, J=7.6 \mathrm{~Hz}, 1 \mathrm{H})$, 6.98-6.89 (m, 2H), $4.02(\mathrm{~s}, 2 \mathrm{H})$ ppm. ${ }^{13} \mathrm{C}\left\{{ }^{1} \mathrm{H}\right\}$ NMR $\left(101 \mathrm{MHz}, \mathrm{CDCl}_{3}, 298 \mathrm{~K}\right): \delta=163.1$ (d, $\left.J_{C, F}=245.8 \mathrm{~Hz}\right), 143.8\left(\mathrm{~d}, J_{\mathrm{C}, \mathrm{F}}=7.0 \mathrm{~Hz}\right), 140.4,129.9\left(\mathrm{~d}, J_{\mathrm{C}, \mathrm{F}}=8.3 \mathrm{~Hz}\right), 129.1,128.7,126.5$, $124.7\left(\mathrm{~d}, J_{\mathrm{C}, \mathrm{F}}=2.3 \mathrm{~Hz}\right), 115.9\left(\mathrm{~d}, J_{\mathrm{C}, \mathrm{F}}=21.3 \mathrm{~Hz}\right), 113.1\left(\mathrm{~d}, J_{\mathrm{C}, \mathrm{F}}=21.4 \mathrm{~Hz}\right), 41.8 \mathrm{ppm} .{ }^{19} \mathrm{~F}$ NMR (471 MHz, $\mathrm{CDCl}_{3}, 298 \mathrm{~K}$ ): $\delta=-113.6 \mathrm{ppm}$. HRMS (APCI): calculated for $\mathrm{C}_{13} \mathrm{H}_{10} \mathrm{~F}^{*+}[\mathrm{M}-\mathrm{H}]^{*+}$ : 185.0767; found 185.0758. The NMR spectroscopic data are in accordance with those reported. ${ }^{19}$

\subsubsection{1-Benzyl-2-fluorobenzene (3ka)}

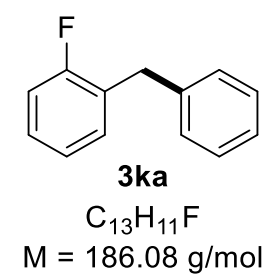

Prepared from 1-(bromomethyl)-2-fluorobenzene (1k, $75.6 \mathrm{mg}, 0.40 \mathrm{mmol}, 1.0$ equiv) using triethyl(phenyl)silane (6a, $92.3 \mathrm{mg}, 0.48 \mathrm{mmol}, 1.2$ equiv) according to GP 2 (>95\% NMR yield). Purification by flash column chromatography on silica gel using $n$-pentane afforded 1-benzyl2-fluorobenzene (3ka) as a colorless oil $\left(60.1 \mathrm{mg}, 81 \%\right.$ yield). $\mathbf{R}_{\boldsymbol{f}}=0.32$ (cyclohexane). ${ }^{1} \mathbf{H}$ NMR (500 MHz, $\left.\mathrm{CDCl}_{3}, 298 \mathrm{~K}\right): \delta=7.32-7.27(\mathrm{~m}, 2 \mathrm{H}), 7.25-7.12(\mathrm{~m}, 5 \mathrm{H}), 7.08-7.01(\mathrm{~m}, 2 \mathrm{H})$, $4.01(\mathrm{~s}, 2 \mathrm{H}) \mathrm{ppm} .{ }^{13} \mathrm{C}\left\{{ }^{1} \mathrm{H}\right\} \mathrm{NMR}\left(101 \mathrm{MHz}, \mathrm{CDCl}_{3}, 298 \mathrm{~K}\right): \delta=161.1\left(\mathrm{~d}, J_{\mathrm{C}, \mathrm{F}}=245.8 \mathrm{~Hz}\right), 140.0$, $131.2\left(\mathrm{~d}, J_{\mathrm{C}, \mathrm{F}}=4.7 \mathrm{~Hz}\right), 128.9,128.6,128.3,128.1\left(\mathrm{~d}, J_{\mathrm{C}, \mathrm{F}}=8.1 \mathrm{~Hz}\right), 126.4,124.2\left(\mathrm{~d}, J_{\mathrm{C}, \mathrm{F}}=\right.$ $3.5 \mathrm{~Hz}), 115.4\left(\mathrm{~d}, \mathrm{~J}_{\mathrm{C}, \mathrm{F}}=22.3 \mathrm{~Hz}\right), 34.9\left(\mathrm{~d}, \mathrm{~J}_{\mathrm{C}, \mathrm{F}}=3.3 \mathrm{~Hz}\right) \mathrm{ppm} .{ }^{19} \mathrm{~F}$ NMR $\left(471 \mathrm{MHz}, \mathrm{CDCl}_{3}, 298\right.$ $\mathrm{K}): \delta=-117.9 \mathrm{ppm}$. HRMS (APCI): calculated for $\mathrm{C}_{13} \mathrm{H}_{10} \mathrm{~F}^{\cdot+}[\mathrm{M}-\mathrm{H}]^{\circ+}$ : 185.0767; found 185.0759. The NMR spectroscopic data are in accordance with those reported. ${ }^{19}$ 


\subsubsection{1-Benzyl-4-chlorobenzene (3la)}

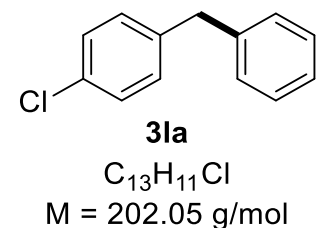

Prepared from 1-(bromomethyl)-4-chlorobenzene (1I, $82.2 \mathrm{mg}, 0.40 \mathrm{mmol}, 1.0$ equiv) using triethyl(phenyl)silane (6a, $92.3 \mathrm{mg}, 0.48 \mathrm{mmol}, 1.2$ equiv) according to GP 2 (>95\% NMR yield). Purification by flash column chromatography on silica gel using $n$-pentane afforded 1-benzyl4-chlorobenzene (3la) as a colorless oil $\left(69.4 \mathrm{mg}, 86 \%\right.$ yield). $\mathbf{R}_{\boldsymbol{f}}=0.32$ (cyclohexane). ${ }^{1} \mathbf{H}$ NMR $\left(500 \mathrm{MHz}, \mathrm{CDCl}_{3}, 298 \mathrm{~K}\right): \delta=7.33-7.28(\mathrm{~m}, 2 \mathrm{H}), 7.28-7.25(\mathrm{~m}, 2 \mathrm{H}), 7.25-7.20(\mathrm{~m}, 1 \mathrm{H})$, 7.20-7.15 (m, 2H), 7.15-7.10 (m, J = 8.5, 2.1 Hz, 2H), $3.96(\mathrm{~s}, 2 \mathrm{H}) \mathrm{ppm} .{ }^{13} \mathrm{C}\left\{{ }^{1} \mathrm{H}\right\}$ NMR $(126$ $\left.\mathrm{MHz}, \mathrm{CDCl}_{3}, 298 \mathrm{~K}\right): \delta=140.7,139.7,132.0,130.4,129.0,128.7$ (2C), 126.4, 41.4 ppm. HRMS (APCI): calculated for $\mathrm{C}_{13} \mathrm{H}_{10} \mathrm{Cl}^{\circ}+[\mathrm{M}-\mathrm{H}]^{*+}$ : 201.0471; found 201.0465. The NMR spectroscopic data are in accordance with those reported. ${ }^{22}$

\subsubsection{1-Benzyl-3-chlorobenzene (3ma)}



Prepared from 1-(bromomethyl)-3-chlorobenzene (1m, $82.2 \mathrm{mg}, 0.40 \mathrm{mmol}, 1.0$ equiv) using triethyl(phenyl)silane (6a, $92.3 \mathrm{mg}, 0.48 \mathrm{mmol}, 1.2$ equiv) according to GP 2 (>95\% NMR yield). Purification by flash column chromatography on silica gel using $n$-pentane afforded 1-benzyl3-chlorobenzene (3ma) as a colorless oil $\left(69.5 \mathrm{mg}, 86 \%\right.$ yield). $\mathbf{R}_{\boldsymbol{f}}=0.31$ (cyclohexane). ${ }^{1} \mathbf{H}$ NMR (500 MHz, $\left.\mathrm{CDCl}_{3}, 298 \mathrm{~K}\right): \delta=7.36-7.28(\mathrm{~m}, 2 \mathrm{H}), 7.26-7.14(\mathrm{~m}, 6 \mathrm{H}), 7.11-7.05(\mathrm{~m}, 1 \mathrm{H})$, 3.97 (s, 2H) ppm. ${ }^{13} \mathrm{C}\left\{{ }^{1} \mathrm{H}\right\}$ NMR $\left(126 \mathrm{MHz}, \mathrm{CDCl}_{3}, 298 \mathrm{~K}\right): \delta=143.3,140.3,134.4,129.8$, 129.2, 129.1, 128.7, 127.2, 126.5, 126.5, 41.7 ppm. HRMS (APCI): calculated for $\mathrm{C}_{13} \mathrm{H}_{10} \mathrm{Cl}^{*+}$ $[\mathrm{M}-\mathrm{H}]^{\circ+}$ : 201.0471; found 201.0466. The NMR spectroscopic data are in accordance with those reported. ${ }^{23}$

3.5.10 1-Benzyl-2-chlorobenzene (3na)

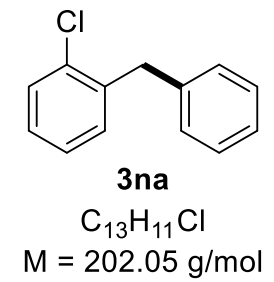

Prepared from 1-(bromomethyl)-2-chlorobenzene (1n, $82.2 \mathrm{mg}, 0.40 \mathrm{mmol}, 1.0$ equiv) using triethyl(phenyl)silane (6a, $92.3 \mathrm{mg}, 0.48 \mathrm{mmol}, 1.2$ equiv) according to GP 2 (>95\% NMR yield). Purification by flash column chromatography on silica gel using $n$-pentane afforded 1-benzyl- 
2-chlorobenzene (3na) as a colorless oil $\left(67.0 \mathrm{mg}, 83 \%\right.$ yield). $\mathbf{R}_{\boldsymbol{f}}=0.31$ (cyclohexane). ${ }^{1} \mathbf{H}$ NMR $\left(500 \mathrm{MHz}, \mathrm{CDCl}_{3}, 298 \mathrm{~K}\right): \delta=7.42-7.37(\mathrm{~m}, 1 \mathrm{H}), 7.34-7.28(\mathrm{~m}, 2 \mathrm{H}), 7.25-7.13(\mathrm{~m}, 6 \mathrm{H})$, $4.13(\mathrm{~s}, 2 \mathrm{H}) \mathrm{ppm} .{ }^{13} \mathrm{C}\left\{{ }^{1} \mathrm{H}\right\}$ NMR $\left(126 \mathrm{MHz}, \mathrm{CDCl}_{3}, 298 \mathrm{~K}\right): \delta=139.7,138.8,134.4,131.2$, 129.7, 129.1, 128.6, 127.8, 127.0, 126.4, 39.3 ppm. HRMS (APCl): calculated for $\mathrm{C}_{13} \mathrm{H}_{10} \mathrm{Cl}^{\circ+}$ $[\mathrm{M}-\mathrm{H}]^{\circ+}:$ 201.0471; found 201.0465. The NMR spectroscopic data are in accordance with those reported. ${ }^{24}$

\subsubsection{1-Benzyl-4-bromobenzene (3oa)}

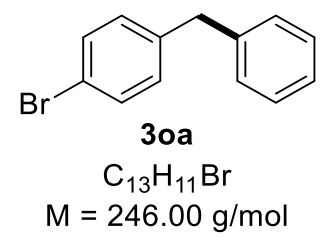

Prepared from 1-bromo-4-(bromomethyl)benzene (10, $100.0 \mathrm{mg}, 0.40 \mathrm{mmol}, 1.0$ equiv) using triethyl(phenyl)silane (6a, $92.3 \mathrm{mg}, 0.48 \mathrm{mmol}, 1.2$ equiv) according to GP 2 (>95\% NMR yield). Purification by flash column chromatography on silica gel using $n$-pentane afforded 1-benzyl4-bromobenzene (30a) as a colorless oil ( $88.1 \mathrm{mg}, 90 \%$ yield). $\mathbf{R}_{\boldsymbol{f}}=0.30$ (cyclohexane). ${ }^{1} \mathbf{H}$ NMR $\left(500 \mathrm{MHz}, \mathrm{CDCl}_{3}, 298 \mathrm{~K}\right): \delta=7.44-7.39(\mathrm{~m}, 2 \mathrm{H}), 7.34-7.28(\mathrm{~m}, 2 \mathrm{H}), 7.25-7.20(\mathrm{~m}, 1 \mathrm{H})$, 7.20-7.14 (m, 2H), 7.10-7.05 (m, 2H), $3.95(\mathrm{~s}, 2 \mathrm{H})$ ppm. ${ }^{13} \mathbf{C}\left\{{ }^{1} \mathrm{H}\right\}$ NMR $\left(126 \mathrm{MHz}, \mathrm{CDCl}_{3}, 298\right.$ $\mathrm{K}): \delta=140.6,140.2,131.6,130.8,129.0,128.7,126.4,120.1,41.5$ ppm. HRMS (APCI): calculated for $\mathrm{C}_{13} \mathrm{H}_{10} \mathrm{Br}^{*+}[\mathrm{M}-\mathrm{H}]^{+*}: 244.9966$; found 244.9960 . The NMR spectroscopic data are in accordance with those reported. ${ }^{23}$

\subsubsection{1-Benzyl-3-bromobenzene (3pa)}

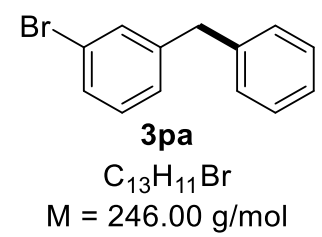

Prepared from 1-bromo-3-(bromomethyl)benzene (1p, $100.0 \mathrm{mg}, 0.40 \mathrm{mmol}, 1.0$ equiv) using triethyl(phenyl)silane (6a, $92.3 \mathrm{mg}, 0.48 \mathrm{mmol}, 1.2$ equiv) according to GP 2 (>95\% NMR yield). Purification by flash column chromatography on silica gel using $n$-pentane afforded 1-benzyl3-bromobenzene (3pa) as a colorless oil $\left(85.1 \mathrm{mg}, 86 \%\right.$ yield). $\mathbf{R}_{\boldsymbol{f}}=0.30$ (cyclohexane). ${ }^{1} \mathbf{H}$ NMR $\left(500 \mathrm{MHz}, \mathrm{CDCl}_{3}, 298 \mathrm{~K}\right): \delta=7.40-7.29(\mathrm{~m}, 4 \mathrm{H}), 7.28-7.22(\mathrm{~m}, 1 \mathrm{H}), 7.22-7.12(\mathrm{~m}, 4 \mathrm{H})$, $3.97(\mathrm{~s}, 2 \mathrm{H}) \mathrm{ppm} .{ }^{13} \mathrm{C}\left\{{ }^{1} \mathrm{H}\right\}$ NMR $\left(126 \mathrm{MHz}, \mathrm{CDCl}_{3}, 298 \mathrm{~K}\right): \delta=143.6,140.3,132.0,130.1$, 129.4, 129.0, 128.7, 127.7, 126.5, 122.7, 41.7 ppm. HRMS (APCI): calculated for $\mathrm{C}_{13} \mathrm{H}_{10} \mathrm{Br}^{*}$ $[\mathrm{M}-\mathrm{H}]^{\circ+}:$ 244.9966, 246.9945; found 244.9961, 246.9939. The NMR spectroscopic data are in accordance with those reported. ${ }^{25}$ 


\subsubsection{1-Benzyl-2-bromobenzene (3qa)}

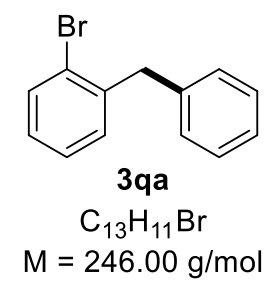

Prepared from 1-bromo-2-(bromomethyl)benzene (1q, $100.0 \mathrm{mg}, 0.40 \mathrm{mmol}, 1.0$ equiv) using triethyl(phenyl)silane (6a, $92.3 \mathrm{mg}, 0.48 \mathrm{mmol}, 1.2$ equiv) according to GP 2 (>95\% NMR yield). Purification by flash column chromatography on silica gel using $n$-pentane afforded 1-benzyl2-bromobenzene (3qa) as a colorless oil ( $87.1 \mathrm{mg}, 88 \%$ yield). $\mathbf{R}_{f}=0.33$ (cyclohexane). ${ }^{1} \mathbf{H}$ NMR $\left(500 \mathrm{MHz}, \mathrm{CDCl}_{3}, 298 \mathrm{~K}\right): \delta=7.55(\mathrm{dd}, J=8.0,1.3 \mathrm{~Hz}, 1 \mathrm{H}), 7.31-7.25(\mathrm{~m}, 2 \mathrm{H}), 7.23-$ $7.15(\mathrm{~m}, 4 \mathrm{H}), 7.11$ (dd, $J=7.6,1.8 \mathrm{~Hz}, 1 \mathrm{H}), 7.06$ (td, $J=7.6,1.8 \mathrm{~Hz}, 1 \mathrm{H}), 4.10(\mathrm{~s}, 2 \mathrm{H}) \mathrm{ppm}$. ${ }^{13} \mathrm{C}\left\{{ }^{1} \mathrm{H}\right\}$ NMR $\left(126 \mathrm{MHz}, \mathrm{CDCl}_{3}, 298 \mathrm{~K}\right): \delta=140.5,139.6,133.0,131.2,129.1,128.6,128.0$, 127.6, 126.4, 125.0, 41.9 ppm. HRMS (APCI): calculated for $\mathrm{C}_{13} \mathrm{H}_{10} \mathrm{Br}^{*+}[\mathrm{M}-\mathrm{H}]^{*+}:$ 244.9966, 246.9945; found 244.9960, 246.9939. The NMR spectroscopic data are in accordance with those reported. ${ }^{23}$

3.5.14 1-Benzyl-4-iodobenzene (3ra)



Prepared from 1-(bromomethyl)-4-iodobenzene (1r, $118.8 \mathrm{mg}, 0.40 \mathrm{mmol}, 1.0$ equiv) using triethyl(phenyl)silane (6a, $92.3 \mathrm{mg}, 0.48 \mathrm{mmol}, 1.2$ equiv) according to GP 2 (94\% NMR yield). Purification by flash column chromatography on silica gel using $n$-pentane afforded 1-benzyl4-iodobenzene (3ra) as a colorless oil ( $101.1 \mathrm{mg}, 86 \%$ yield). $\mathbf{R}_{f}=0.31$ (cyclohexane). ${ }^{1} \mathbf{H}$ NMR $\left(400 \mathrm{MHz}, \mathrm{CDCl}_{3}, 298 \mathrm{~K}\right): \delta=7.60(\mathrm{~d}, J=8.2 \mathrm{~Hz}, 2 \mathrm{H}), 7.33-7.27(\mathrm{~m}, 2 \mathrm{H}), 7.25-7.19(\mathrm{~m}, 1 \mathrm{H})$, $7.16(\mathrm{~d}, J=7.4 \mathrm{~Hz}, 2 \mathrm{H}), 6.95(\mathrm{~d}, J=8.0 \mathrm{~Hz}, 2 \mathrm{H}), 3.93(\mathrm{~s}, 2 \mathrm{H}) \mathrm{ppm} .{ }^{13} \mathrm{C}\left\{{ }^{1} \mathrm{H}\right\} \mathbf{N M R}(101 \mathrm{MHz}$, $\left.\mathrm{CDCl}_{3}, 298 \mathrm{~K}\right): \delta=140.9,140.5,137.6,131.1,129.0,128.7,126.4,91.4,41.5$ ppm. HRMS (APCI): calculated for $\mathrm{C}_{13} \mathrm{H}_{11} 1^{\circ+}[\mathrm{M}]^{*+}:$ 293.9905; found 293.9901. The NMR spectroscopic data are in accordance with those reported. ${ }^{26}$

\subsubsection{Cyclopentylbenzene (3sa)}




Prepared from bromocyclopentane (1s, $59.6 \mathrm{mg}, 0.40 \mathrm{mmol}, 1.0$ equiv) according to GP 3 (94\% NMR yield). Purification by flash column chromatography on silica gel using $n$-pentane afforded cyclopentylbenzene (3sa) as a colorless oil (38.9 $\mathrm{mg}, 67 \%$ yield). $\mathbf{R}_{\boldsymbol{f}}=0.61$ (cyclohexane). ${ }^{1} \mathrm{H}$ NMR (500 MHz, $\left.\mathrm{CDCl}_{3}, 298 \mathrm{~K}\right): \delta=7.31-7.21(\mathrm{~m}, 4 \mathrm{H}), 7.20-7.12(\mathrm{~m}, 1 \mathrm{H})$, 3.05-2.92 (m, 1H), 2.15-1.98 (m, 2H), 1.84-1.75 (m, 2H), 1.73-1.64 (m, 2H), 1.64-1.54 (m, $2 \mathrm{H}) \mathrm{ppm} .{ }^{13} \mathrm{C}\left\{{ }^{1} \mathrm{H}\right\}$ NMR $\left(126 \mathrm{MHz}, \mathrm{CDCl}_{3}, 298 \mathrm{~K}\right): \delta=146.7,128.4,127.2,125.8,46.1,34.7$, 25.7 ppm. HRMS (APCl): calculated for $\mathrm{C}_{11} \mathrm{H}_{13^{*}}[\mathrm{M}-\mathrm{H}]^{++}$: 145.1017 ; found 145.1012. The NMR spectroscopic data are in accordance with those reported. ${ }^{27}$

3.5.16 Cyclohexylbenzene (3ta)

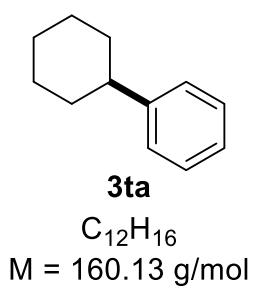

Prepared from bromocyclohexane (1t, $65.2 \mathrm{mg}, 0.40 \mathrm{mmol}, 1.0$ equiv) according to GP 3 (90\% NMR yield). Purification by flash column chromatography on silica gel using $n$-pentane afforded cyclohexylbenzene (3ta) as a colorless oil $\left(46.6 \mathrm{mg}, 74 \%\right.$ yield). $\mathbf{R}_{\boldsymbol{f}}=0.60$ (cyclohexane). ${ }^{1} \mathrm{H}$ NMR (500 MHz, $\left.\mathrm{CDCl}_{3}, 298 \mathrm{~K}\right): \delta=7.34-7.27(\mathrm{~m}, 2 \mathrm{H}), 7.24-7.16(\mathrm{~m}, 3 \mathrm{H})$, 2.59-2.44 (t, $J=11.5,3.5 \mathrm{~Hz}, 1 \mathrm{H}), 1.96-1.80(\mathrm{~m}, 4 \mathrm{H}), 1.80-1.71(\mathrm{~m}, 1 \mathrm{H}), 1.50-1.34(\mathrm{~m}, 4 \mathrm{H})$, 1.35-1.20 (m, 1H) ppm. ${ }^{13} \mathrm{C}\left\{{ }^{1} \mathrm{H}\right\}$ NMR $\left(126 \mathrm{MHz}, \mathrm{CDCl}_{3}, 298 \mathrm{~K}\right): \delta=148.2,128.4,127.0$, 125.9, 44.8, 34.6, 27.1, 26.3 ppm. HRMS (APCI): calculated for $\mathrm{C}_{12} \mathrm{H}_{15^{*}}[\mathrm{M}-\mathrm{H}]^{0+*}$ : 159.1174; found 159.1167. The NMR spectroscopic data are in accordance with those reported. ${ }^{17}$

\subsubsection{2-Phenyladamantane (3ua)}



Prepared from 2-bromoadamantane (1u, $86.1 \mathrm{mg}, 0.40 \mathrm{mmol}, 1.0$ equiv) using triethyl(phenyl)silane (6a, $92.3 \mathrm{mg}, 0.48 \mathrm{mmol}, 1.2$ equiv) according to GP 2 (94\% NMR yield). Purification by flash column chromatography on silica gel using $n$-pentane afforded 2-phenyladamantane (3ua) as a colorless oil $\left(76.4 \mathrm{mg}, 90 \%\right.$ yield). $\mathbf{R}_{\boldsymbol{f}}=0.55$ (cyclohexane). ${ }^{1} \mathbf{H}$ NMR $\left(500 \mathrm{MHz}, \mathrm{CDCl}_{3}, 298 \mathrm{~K}\right): \delta=7.39-7.30(\mathrm{~m}, 4 \mathrm{H}), 7.21-7.15(\mathrm{~m}, 1 \mathrm{H}), 3.02(\mathrm{~s}, 1 \mathrm{H}), 2.48(\mathrm{~s}, 2 \mathrm{H})$, 2.06-1.91 (m, 5H), $1.86(\mathrm{~d}, J=12.4 \mathrm{~Hz}, 2 \mathrm{H}), 1.78(\mathrm{~s}, 3 \mathrm{H}), 1.57$ (d, $J=12.5 \mathrm{~Hz}, 2 \mathrm{H})$ ppm. ${ }^{13} \mathrm{C}\left\{{ }^{1} \mathrm{H}\right\}$ NMR $\left(126 \mathrm{MHz}, \mathrm{CDCl}_{3}, 298 \mathrm{~K}\right): \delta=144.6,128.3,127.0,125.3,47.0,39.4,38.1,32.1$, 31.2, 28.2, 28.0 ppm. HRMS (APCI): calculated for $\mathrm{C}_{16} \mathrm{H}_{19^{*}}[\mathrm{M}-\mathrm{H}]^{\circ+}$ : 211.1487; found 211.1480. The NMR spectroscopic data are in accordance with those reported. ${ }^{28}$ 
3.5.18 tert-Butylbenzene (3va)

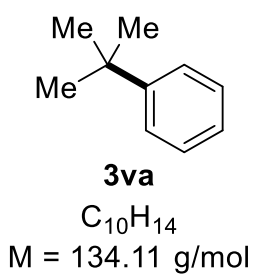

Prepared from 2-bromo-2-methylpropane (1v, $54.8 \mathrm{mg}, 0.40 \mathrm{mmol}, 1.0$ equiv) according to GP 3 (>95\% NMR yield). Due to the low boiling point, this product was not isolated. Selected NMR spectroscopic data for $3 \mathbf{v a}$ from the crude reaction mixture: ${ }^{1} \mathbf{H}$ NMR $\left(500 \mathrm{MHz}, \mathrm{C}_{6} \mathrm{D}_{6}, 298 \mathrm{~K}\right)$ : $\delta=1.22(\mathrm{~s}, 9 \mathrm{H}) \mathrm{ppm}$. The NMR spectroscopic data are in accordance with those reported. ${ }^{29}$

3.5.19 1-Phenyladamantane (3wa)

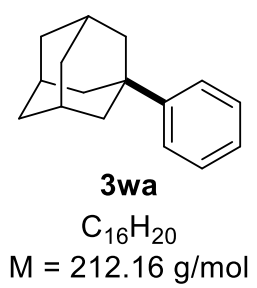

Prepared from 1-bromoadamantane (1w, $86.1 \mathrm{mg}, 0.40 \mathrm{mmol}, 1.0$ equiv) according to GP $\mathbf{3}$ ( $90 \%$ NMR yield). Purification by flash column chromatography on silica gel using $n$-pentane afforded 1-phenyladamantane (3wa) as a white solid (70.4 mg, $83 \%$ yield). $\mathbf{R}_{\boldsymbol{f}}=0.55$ (cyclohexane). ${ }^{1} \mathrm{H}$ NMR (400 MHz, $\left.\mathrm{CDCl}_{3}, 298 \mathrm{~K}\right): \delta=7.42-7.38(\mathrm{~m}, 2 \mathrm{H}), 7.37-7.32(\mathrm{~m}, 2 \mathrm{H})$, 7.23-7.17 (m, 1H), 2.13, (s, 3H), $1.94(\mathrm{~d}, J=2.9 \mathrm{~Hz}, 6 \mathrm{H}), 1.87-1.74(\mathrm{~m}, 6 \mathrm{H}) \mathrm{ppm} .{ }^{13} \mathrm{C}\left\{{ }^{1} \mathrm{H}\right\}$ NMR (101 MHz, $\left.\mathrm{CDCl}_{3}, 298 \mathrm{~K}\right): \delta=151.4,128.2,125.6,125.0,43.3,37.0,36.3,29.1$ ppm. HRMS (APCI): calculated for $\mathrm{C}_{16} \mathrm{H}_{19^{+}}[\mathrm{M}-\mathrm{H}]^{++}$: 211.1487; found 211.1479. The NMR spectroscopic data are in accordance with those reported. ${ }^{30}$

3.5.20 1,3,5-Trimethyl-2-(3-phenylpropyl)benzene (3cb)

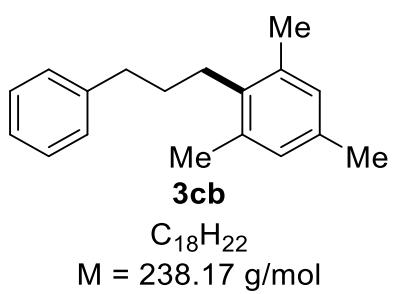

Prepared from (3-bromopropyl)benzene (1c, $79.6 \mathrm{mg}, 0.40 \mathrm{mmol}, 1.0$ equiv) and mesitylene (2b, 240.4 mg, 2.0 mmol, 5.0 equiv) using triethyl(phenyl)silane (6a, 92.3 mg, 0.48 mmol, 1.2 equiv) according to GP 4 (93\% NMR yield). Purification by flash column chromatography on silica gel using $n$-pentane/EtOAc (50:1) afforded 1,3,5-trimethyl-2-(3-phenylpropyl)benzene (3cb) as a colorless oil $\left(76.1 \mathrm{mg}, 80 \%\right.$ yield). $\mathbf{R}_{\boldsymbol{f}}=0.15$ (cyclohexane). ${ }^{1} \mathbf{H}$ NMR $(500 \mathrm{MHz}$, $\left.\mathrm{CDCl}_{3}, 298 \mathrm{~K}\right): \delta=7.33-7.27(\mathrm{~m}, 2 \mathrm{H}), 7.25-7.18(\mathrm{~m}, 3 \mathrm{H}), 6.83(\mathrm{~s}, 2 \mathrm{H}), 2.75(\mathrm{t}, J=7.6 \mathrm{~Hz}, 2 \mathrm{H})$, 2.65-2.56 (m, 2H), $2.25(\mathrm{~s}, 3 \mathrm{H}), 2.24(\mathrm{~s}, 6 \mathrm{H}), 1.84-1.73(\mathrm{~m}, 2 \mathrm{H}) \mathrm{ppm} .{ }^{13} \mathrm{C}\left\{{ }^{1} \mathrm{H}\right\} \mathbf{N M R}(126 \mathrm{MHz}$, 
$\left.\mathrm{CDCl}_{3}, 298 \mathrm{~K}\right): \delta=142.4,136.3,136.0,135.0,129.0,128.5,128.4,125.9,36.5,30.8,29.1$, 20.9, 19.8 ppm. HRMS (APCI): calculated for $\mathrm{C}_{18} \mathrm{H}_{22}{ }^{*+}[\mathrm{M}]^{\circ+}:$ 238.1722; found 238.1717. The NMR spectroscopic data are in accordance with those reported. ${ }^{31}$

3.5.21 2-Benzyl-1,3,5-trimethylbenzene (3eb)



Prepared from benzylbromide (1e, $68.4 \mathrm{mg}, 0.40 \mathrm{mmol}, 1.0$ equiv) and mesitylene (2b, 240.4 mg, 2.0 mmol, 5.0 equiv) according to GP 4 (90\% NMR yield). Purification by flash column chromatography on silica gel using $n$-pentane afforded 2-benzyl-1,3,5-trimethylbenzene (3eb) as a colorless oil (57.2 mg, 68\% yield). $\mathbf{R}_{\boldsymbol{f}}=0.28$ (cyclohexane). ${ }^{1} \mathbf{H}$ NMR (500 MHz, $\mathrm{CDCl}_{3}$, $298 \mathrm{~K}$ ): $\delta=7.27$ (d, J = 7.9 Hz, 2H), 7.22-7.16 (m, 1H), $7.06(\mathrm{~d}, J=8.1 \mathrm{~Hz}, 2 \mathrm{H}), 6.94(\mathrm{~s}, 2 \mathrm{H})$, $4.07(\mathrm{~s}, 2 \mathrm{H}), 2.34(\mathrm{~s}, 3 \mathrm{H}), 2.25(\mathrm{~s}, 6 \mathrm{H}) \mathrm{ppm} .{ }^{13} \mathrm{C}\left\{{ }^{1} \mathrm{H}\right\} \mathrm{NMR}\left(126 \mathrm{MHz}, \mathrm{CDCl}_{3}, 298 \mathrm{~K}\right): \delta=140.2$, 137.2, 135.8, 133.9, 129.0, 128.5, 128.0, 125.8, 34.9, 21.1, 20.3 ppm. HRMS (APCI): calculated for $\mathrm{C}_{16} \mathrm{H}_{17^{*}}[\mathrm{M}-\mathrm{H}]^{\circ+}$ : 209.1330; found 209.1325. The NMR spectroscopic data are in accordance with those reported. ${ }^{32}$

3.5.22 2-Benzyl-1,4-dimethylbenzene (3ec)

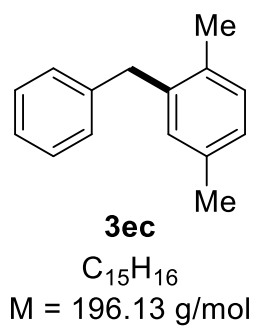

Prepared from benzylbromide (1e, $68.4 \mathrm{mg}, 0.40 \mathrm{mmol}, 1.0$ equiv) and $p$-xylene (2c, $212.3 \mathrm{mg}$, 2.0 mmol. 5.0 equiv) using triethyl(phenyl)silane (6a, $92.3 \mathrm{mg}, 0.48 \mathrm{mmol}, 1.2$ equiv) according to GP 4 (90\% NMR yield). Purification by flash column chromatography on silica gel using $n$ pentane afforded 2-benzyl-1,4-dimethylbenzene (3ec) as a colorless oil (53.2 mg, $68 \%$ ). The isolated product $3 \mathrm{ec}$ was contaminated with traces of diphenylmethane. $\mathbf{R}_{\boldsymbol{f}}=0.24$ (cyclohexane). ${ }^{1} \mathrm{H}$ NMR $\left(400 \mathrm{MHz}, \mathrm{CDCl}_{3}, 298 \mathrm{~K}\right): \delta=7.34-7.27(\mathrm{~m}, 2 \mathrm{H}), 7.25-7.18(\mathrm{~m}, 1 \mathrm{H})$, 7.18-7.14 (m, 2H), 7.09 (d, J = 7.6 Hz, 1H), 7.00 (dd, $J=7.6,1.9 \mathrm{~Hz}, 1 \mathrm{H}), 6.97(\mathrm{~d}, J=1.8 \mathrm{~Hz}$, $1 \mathrm{H}), 3.99(\mathrm{~s}, 2 \mathrm{H}), 2.33(\mathrm{~s}, 3 \mathrm{H}), 2.24(\mathrm{~s}, 3 \mathrm{H}) \mathrm{ppm} .{ }^{13} \mathrm{C}\left\{{ }^{1} \mathrm{H}\right\}$ NMR $\left(101 \mathrm{MHz}, \mathrm{CDCl}_{3}, 298 \mathrm{~K}\right): \delta=$ 140.7, 138.8, 135.5, 133.6, 130.9, 130.3, 128.8, 128.5, 127.2, 126.0, 39.6, 21.1, 19.3 ppm. HRMS (APCl): calculated for $\mathrm{C}_{15} \mathrm{H}_{15}{ }^{+}[\mathrm{M}-\mathrm{H}]^{*+}$ : 195.1174; found 195.1169. The NMR spectroscopic data are in accordance with those reported. ${ }^{33}$ 
3.5.23 Silylium-Ion-Promoted Friedel-Crafts Alkylation of Toluene (2d) with Benzylbromide (1e)

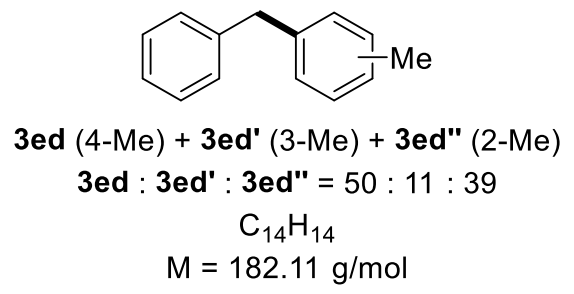

The Friedel-Crafts benzylation of toluene $(\mathbf{2 d}, 184.3 \mathrm{mg}, 2.0 \mathrm{mmol}$. 5.0 equiv) with benzylbromide (1e, $68.4 \mathrm{mg}, 0.40 \mathrm{mmol}, 1.0$ equiv) using triethyl(tolyl)silane (6b, $99.0 \mathrm{mg}, 0.48$ mmol, 1.2 equiv) was performed according to GP 4 (90\% NMR yield). Purification by flash column chromatography on silica gel using $n$-pentane afforded a regioisomeric mixture of $3 \mathbf{e d}$, 3ed' and 3ed" as a colorless oil (46.5 mg, 64\% yield, 3ed:3ed':3ed" = 50:11:39). The regioisomeric ratio was determined by ${ }^{1} \mathrm{H}$ NMR spectroscopy as well as GLC analysis after purification. For the characterization data of $3 \mathbf{e d}(=3 \mathbf{f a})$ see section 3.5 .2 ; for the characterization data of $\mathbf{3 e d}$ ' (= $\mathbf{3 g a}$ ), see section 3.5.3; for the characterization data of $\mathbf{3 e d "}$ (= 3ha), see section 3.5.4.

3.5.24 Silylium-lon-Promoted Friedel-Crafts Alkylation of Naphthalene (2e) with Benzylbromide (1e)



The Friedel-Crafts benzylation of naphthalene (2e, $256.1 \mathrm{mg}, 2.0 \mathrm{mmol}$. 5.0 equiv) with benzylbromide (1e, $68.4 \mathrm{mg}, 0.40 \mathrm{mmol}, 1.0$ equiv) using triethyl(phenyl)silane (6a, $92.3 \mathrm{mg}$, $0.48 \mathrm{mmol}, 1.2$ equiv) was performed according to GP 4 ( $80 \%$ NMR yield). Purification by flash column chromatography on silica gel using $n$-pentane afforded a regioisomeric mixture of 1 benzylnaphthalene (3ee) and 2-benzylnaphthalene (3ee') as a colorless oil (56.0 mg, 64\% yield, 3ee:3ee' $=62: 38)$. The regioisomeric ratio was determined by ${ }^{1} \mathrm{H}$ NMR spectroscopy after purification. Selected NMR spectroscopic data for 1-benzylnaphthalene (3ee): ${ }^{1} \mathbf{H}$ NMR $\left(400 \mathrm{MHz}, \mathrm{CDCl}_{3}, 298 \mathrm{~K}\right): \delta=8.00-7.96(\mathrm{~m}, 1 \mathrm{H}), 7.85-7.81(\mathrm{~m}, 1 \mathrm{H}), 4.45(\mathrm{~s}, 2 \mathrm{H}) \mathrm{ppm}$. The NMR spectroscopic data are in accordance with those reported. ${ }^{34}$ Selected NMR spectroscopic data for 2-benzylnaphthalene (3ee'): ${ }^{1} \mathbf{H}$ NMR $\left(400 \mathrm{MHz}, \mathrm{CDCl}_{3}, 298 \mathrm{~K}\right): \delta=7.63$ (s, 1H), $4.14(\mathrm{~s}, 2 \mathrm{H})$ ppm. (HRMS (APCl): calculated for $\mathrm{C}_{17} \mathrm{H}_{13}{ }^{*+}[\mathrm{M}-\mathrm{H}]^{\circ+}:$ 217.1017; found 217.1013. The NMR spectroscopic data are in accordance with those reported. ${ }^{35}$ 
3.5.25 Ethylbenzene (3xa)



Prepared from bromoethane (1x, $21.8 \mathrm{mg}, 0.20 \mathrm{mmol}, 1.0$ equiv) using triethyl(phenyl)silane (6a, $46.2 \mathrm{mg}, 0.24 \mathrm{mmol}, 1.2$ equiv) according to GP 2 (85\% NMR yield). Selected NMR spectroscopic data: ${ }^{1} \mathrm{H}$ NMR $\left(500 \mathrm{MHz}, \mathrm{C}_{6} \mathrm{D}_{6}, 298 \mathrm{~K}\right): \delta=2.46(\mathrm{q}, J=7.6 \mathrm{~Hz}, 2 \mathrm{H}), 1.09$ (t, $J=$ 7.6 Hz, 3H) ppm. No aromatic $\mathrm{C}-\mathrm{H}$ resonances were detected because of quantitative $\mathrm{H} / \mathrm{D}$ exchange after addition of $\mathrm{C}_{6} \mathrm{D}_{6}$ for NMR spectroscopic analysis.

3.5.26 Diphenylmethane (8)

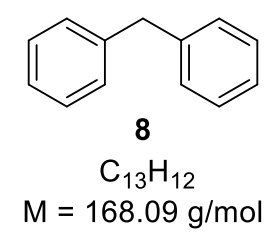

Prepared from dibromethane $(7,34.8 \mathrm{mg}, 0.20 \mathrm{mmol}, 1.0$ equiv) using triethyl(phenyl)silane (6a, $84.6 \mathrm{mg}, 0.44 \mathrm{mmol}, 2.2$ equiv) according to GP 2 (88\% NMR yield). For the characterization data of 8 (= 3ea), see section 3.5.1.

3.5.27 1,2,3,4-Tetrahydronaphthalene (10)

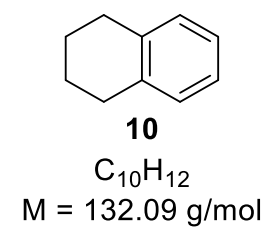

Preparation by intermolecular Friedel-Crafts alkylation of benzene with 1,4-dibromobutane (9): Prepared from 1,4-dibromobutane $(9,43.2 \mathrm{mg}, 0.20 \mathrm{mmol}, 1.0$ equiv) using triethyl(phenyl)silane (6a, $84.6 \mathrm{mg}, 0.44 \mathrm{mmol}, 2.2$ equiv) according to GP 2 (45\% NMR yield). For selected characterization data of $\mathbf{1 0}$, see below.

Preparation by intramolecular Friedel-Crafts alkylation of (4-bromobutyl)benzene (1y):

Prepared from (4-bromobutyl)benzene (1y, $42.6 \mathrm{mg}, 0.20 \mathrm{mmol}, 1.0$ equiv) and triethyl(phenyl)silane (6a, $46.2 \mathrm{mg}, 0.24 \mathrm{mmol}, 1.2$ equiv) according to GP 2 (>95\% NMR yield). Selected NMR spectroscopic data for 10: ${ }^{1} \mathrm{H}$ NMR $\left(500 \mathrm{MHz}, \mathrm{C}_{6} \mathrm{D}_{6}, 298 \mathrm{~K}\right): \delta=2.61-2.55(\mathrm{~m}$, $2 \mathrm{H})$, 1.57-1.51 (m, 2H) ppm. No aromatic $\mathrm{C}-\mathrm{H}$ resonances were detected because of quantitative $H / D$ exchange after addition of $\mathrm{C}_{6} \mathrm{D}_{6}$ for NMR spectroscopic analysis. HRMS (APCI): calculated for $\mathrm{C}_{10} \mathrm{H}_{11}{ }^{+}[\mathrm{M}-\mathrm{H}]^{++}$: 131.0861; found 131.0854. The NMR spectroscopic data are in accordance with those reported. ${ }^{36}$ 


\section{Experimental Details of the Mechamistic Control Experiments}

4.1 Protodesilylation of Triethyl(phenyl)silane (6a) with Reed's Benzenium Carborate $\left[\mathrm{C}_{6} \mathrm{H}_{7}\right]^{+}\left[\mathrm{HCB}_{11} \mathrm{H}_{5} \mathrm{Br}_{6}\right]^{-}$

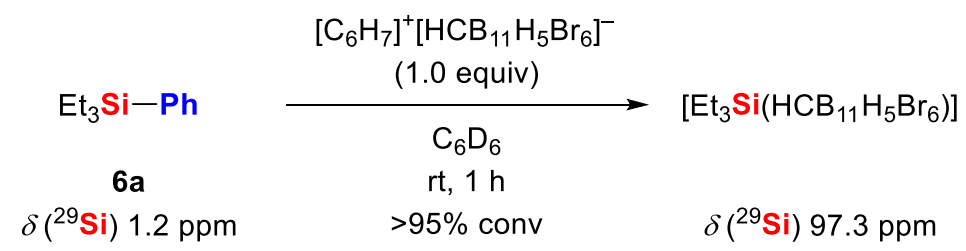

In a glovebox, Reed's benzenium salt $\left[\mathrm{C}_{6} \mathrm{H}_{7}\right]^{+}\left[\mathrm{HCB}_{11} \mathrm{H}_{5} \mathrm{Br}_{6}\right]^{-}$(13.9 mg, $0.02 \mathrm{mmol}, 1.0$ equiv) was suspended in benzene- $d_{6}(0.1 \mathrm{~mL})$. A solution of triethyl(phenyl)silane $(6 \mathbf{a}, 3.8 \mathrm{mg}, 0.02$ mmol, 1.0 equiv) in benzene- $d_{6}(0.4 \mathrm{~mL})$ was added dropwise, and the resulting mixture was stirred at ambient temperature for $1 \mathrm{~h}$. The reaction mixture was directly subjected to NMR spectroscopic analysis, revealing the formation of $\left[\mathrm{Et}_{3} \mathrm{Si}\left(\mathrm{HCB}_{11} \mathrm{H}_{5} \mathrm{Br}_{6}\right)\right]$ (Figure S2).


Figure S2. $\quad{ }^{1} \mathbf{H} /{ }^{29} \mathrm{Si} \mathrm{HMQC} \mathrm{NMR} \mathrm{spectrum}\left(500 / 99 \mathrm{MHz}, \mathrm{C}_{6} \mathrm{D}_{6}, 298 \mathrm{~K}\right.$, optimized for $\left.\mathrm{J}=7 \mathrm{~Hz}\right)$ of triethyl(phenyl)silane (6a) (top) and after reaction with $\left[\mathrm{C}_{6} \mathrm{H}_{7}\right]^{+}\left[\mathrm{HCB}_{11} \mathrm{H}_{5} \mathrm{Br}_{6}\right]^{-}$ (bottom). ( ${ }^{*}$ for $\left[\mathrm{Et}_{3} \mathrm{Si}\left(\mathrm{HCB}_{11} \mathrm{H}_{5} \mathrm{Br}_{6}\right)\right]$ and $v$ for its water adduct) 


\subsection{Investigation of the Defunctionalization}

\subsubsection{Preparation of 1-Benzyl-4-bromobenzene (3oa) on Larger Scale}

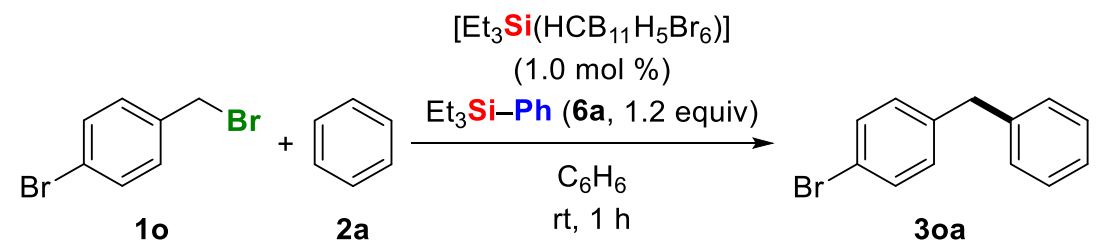

In a glovebox, silylium carborate $\left[\mathrm{Et}_{3} \mathrm{Si}\left(\mathrm{HCB}_{11} \mathrm{H}_{5} \mathrm{Br}_{6}\right)\right](14.6 \mathrm{mg}, 0.02 \mathrm{mmol}, 1.0 \mathrm{~mol} \%)$ was suspended in benzene $(1.0 \mathrm{~mL})$ and treated with triethyl(phenyl)silane $(6 \mathbf{a}, 461.6 \mathrm{mg}, 2.4$ mmol, 1.2 equiv). A solution of 4-bromobenzylbromide (10, 499.9 mg, 2.0 mmol, 1.0 equiv) in benzene $(4.0 \mathrm{~mL})$ was added dropwise, and the resulting mixture was stirred at ambient temperature for $1 \mathrm{~h}$. Cyclohexane $(110 \mu \mathrm{L}, 1.0 \mathrm{mmol}, 0.5$ equiv) was added as an internal standard to determine the yield by ${ }^{1} \mathrm{H}$ NMR spectroscopy (>95\%). Purification by flash column chromatography on silica gel using $n$-pentane as eluent afforded 1-benzyl-4-bromobenzene (3oa) in analytically pure form ( $446.5 \mathrm{mg}, 91 \%$ yield). For the characterization data of $30 a$, see section 3.5.11.

4.2.2 Defunctionalization of 1-Benzyl-4-bromobenzene (3oa)

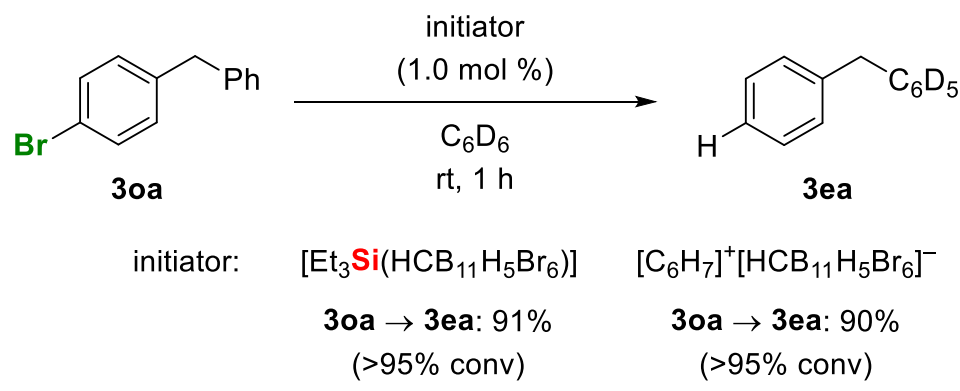

In a glovebox, silylium carborate $\left[\mathrm{Et}_{3} \mathrm{Si}\left(\mathrm{HCB}_{11} \mathrm{H}_{5} \mathrm{Br}_{6}\right)\right](1.5 \mathrm{mg}, 0.002 \mathrm{mmol}, 1.0 \mathrm{~mol} \%)$ and Reed's benzenium salt $\left[\mathrm{C}_{6} \mathrm{H}_{7}\right]^{+}\left[\mathrm{HCB}_{11} \mathrm{H}_{5} \mathrm{Br}_{6}\right]^{-}(1.4 \mathrm{mg}, 0.002 \mathrm{mmol}, 1.0 \mathrm{~mol} \%)$, respectively, was suspended in benzene- $d_{6}(0.1 \mathrm{~mL})$. A solution of 1-benzyl-4-bromobenzene (30a, $492 \mathrm{mg}$, $0.2 \mathrm{mmol}, 1.0$ equiv) in benzene- $d_{6}(0.4 \mathrm{~mL})$ was added dropwise, and the resulting mixture was stirred at ambient temperature for $1 \mathrm{~h}$. Cyclohexane $(22.0 \mu \mathrm{L}, 0.2 \mathrm{mmol}, 1.0$ equiv) was added as an internal standard, and the reaction mixture was directly subjected to NMR spectroscopic analysis. Almost complete conversion of 1-benzyl-4-bromobenzene (3oa) was detected (>95\%), and the formation of diphenylmethane (3ea) as the main product was observed (91\% NMR yield using $\left[\mathrm{Et}_{3} \mathrm{Si}\left(\mathrm{HCB}_{11} \mathrm{H}_{5} \mathrm{Br}_{6}\right)\right]$ as initiator; 90\% NMR yield using $\left[\mathrm{C}_{6} \mathrm{H}_{7}\right]^{+}\left[\mathrm{HCB}_{11} \mathrm{H}_{5} \mathrm{Br}_{6}\right]^{-}$as initiator). For the characterization data of 3ea, see section 3.5.1. Note: No aromatic $\mathrm{C}-\mathrm{H}$ resonances were detected because of quantitative $\mathrm{H} / \mathrm{D}$ exchange after addition of $C_{6} D_{6}$ for NMR spectroscopic analysis. 
4.2.3 Monitoring the Friedel-Crafts Alkylation of Benzene with 1-(Bromomethyl)-4fluorobenzene (1i) by ${ }^{19} \mathrm{~F}$ NMR Spectroscopy

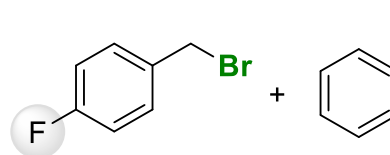

1i



$\mathrm{rt}, 1 \mathrm{~h}$

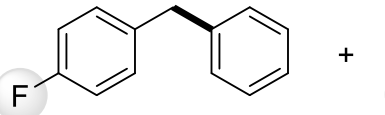

3ia

$$
\delta\left({ }^{19} \mathrm{~F}\right)-117.2 \mathrm{ppm} \quad \delta\left({ }^{19} \mathrm{~F}\right)-112.8 \mathrm{ppm}
$$<smiles>C[14c]1ccc(Cc2ccccc2)cc1</smiles>

In a glovebox, silylium carborate $\left[\mathrm{Et}_{3} \mathrm{Si}\left(\mathrm{HCB}_{11} \mathrm{H}_{5} \mathrm{Br}_{6}\right)\right](1.5 \mathrm{mg}, 0.002 \mathrm{mmol}, 1.0 \mathrm{~mol} \%)$ was suspended in benzene- $d_{6}(0.1 \mathrm{~mL})$. A solution of 1-(bromomethyl)-4-fluorobenzene (1i, 37.8 $\mathrm{mg}, 0.2 \mathrm{mmol}, 1.0$ equiv) in benzene- $d_{6}(0.4 \mathrm{~mL})$ was added dropwise, and the resulting mixture was stirred at ambient temperature for $1 \mathrm{~h}$. Cyclohexane ( $22.0 \mu \mathrm{L}, 0.2 \mathrm{mmol}, 1.0$ equiv) was added as an internal standard, and the reaction mixture was directly subjected to NMR spectroscopic analysis. Complete conversion of 1-(bromomethyl)-4-fluorobenzene (1i) was detected (>95\%), and the formation of 1-benzyl-4-fluorobenzene (3ia) (46\% NMR yield) and fluorobenzene (S6) was observed by ${ }^{19} \mathrm{~F}$ NMR spectroscopy (Figure S3). The formation of diphenylmethane (3ea) as the main product (50\% NMR yield) was verified by ${ }^{1} \mathrm{H}$ NMR spectroscopy. Note: Because of quantitative $H / D$ exchange with $\mathrm{C}_{6} \mathrm{D}_{6}$ as solvent, all aryl groups are in fact fully deuterated.

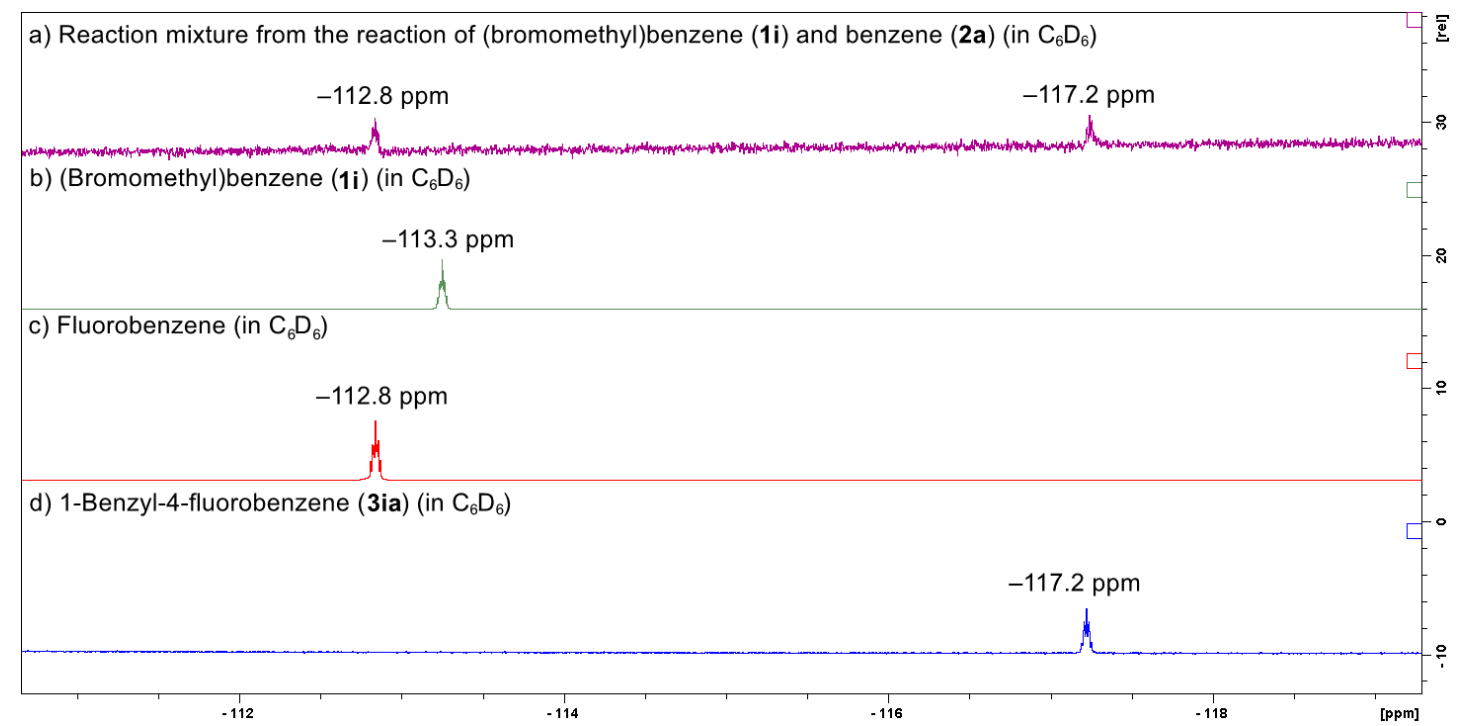

Figure S3. Selected segment of the ${ }^{19} \mathrm{~F}$ NMR spectra $\left(471 \mathrm{MHz}, \mathrm{C}_{6} \mathrm{D}_{6}, 298 \mathrm{~K}\right)$ for the FriedelCrafts benzylation of benzene with 1-(bromomethyl)-4-fluorobenzene (1i): a) ${ }^{19} \mathrm{~F}$ NMR spectra of the crude reaction mixture; b) ${ }^{19} \mathrm{~F}$ NMR spectra of pure 1(bromomethyl)-4-fluorobenzene (1i); c) ${ }^{19} \mathrm{~F}$ NMR spectra of pure fluorobenzene (S6); d) ${ }^{19} \mathrm{~F}$ NMR spectra of pure 1-benzyl-4-fluorobenzene (3ia). 
4.2.4 Proposed Mechanism for the Catalytic Defunctionalization (Debenzylation)

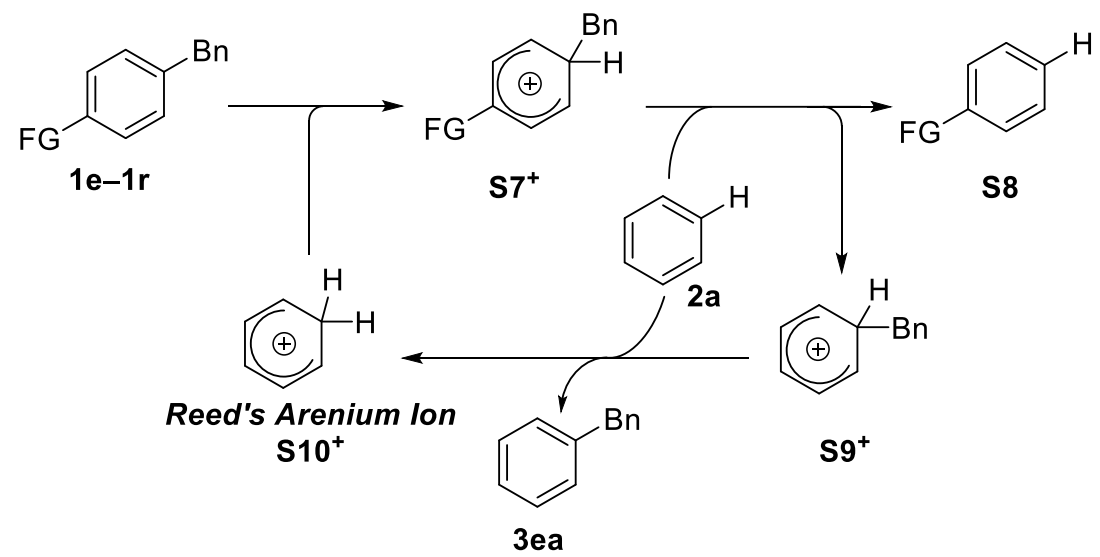

Figure S4. Plausible catalytic cycle for the defunctionalization (debenzylation). The counteranion $\left.\left[\mathrm{HCB}_{11} \mathrm{H}_{5} \mathrm{Br}_{6}\right)\right]^{-}$is omitted for clarity. 


\section{$5 \quad$ NMR Spectra}

Figure S5. ${ }^{1} \mathbf{H}$ NMR spectrum $\left(500 \mathrm{MHz}, \mathrm{CDCl}_{3}, 298 \mathrm{~K}\right)$ of 3ea from the reaction of benzyl bromide (1e) and benzene (2a).


3ea




Figure S6. $\quad{ }^{13} \mathbf{C}\left\{{ }^{1} \mathrm{H}\right\}$ NMR spectrum $\left(126 \mathrm{MHz} \mathrm{CDCl}_{3}, 298 \mathrm{~K}\right)$ of 3ea from the reaction of benzyl bromide (1e) and benzene (2a).

$$
\left.\right|^{\stackrel{m}{+}}
$$
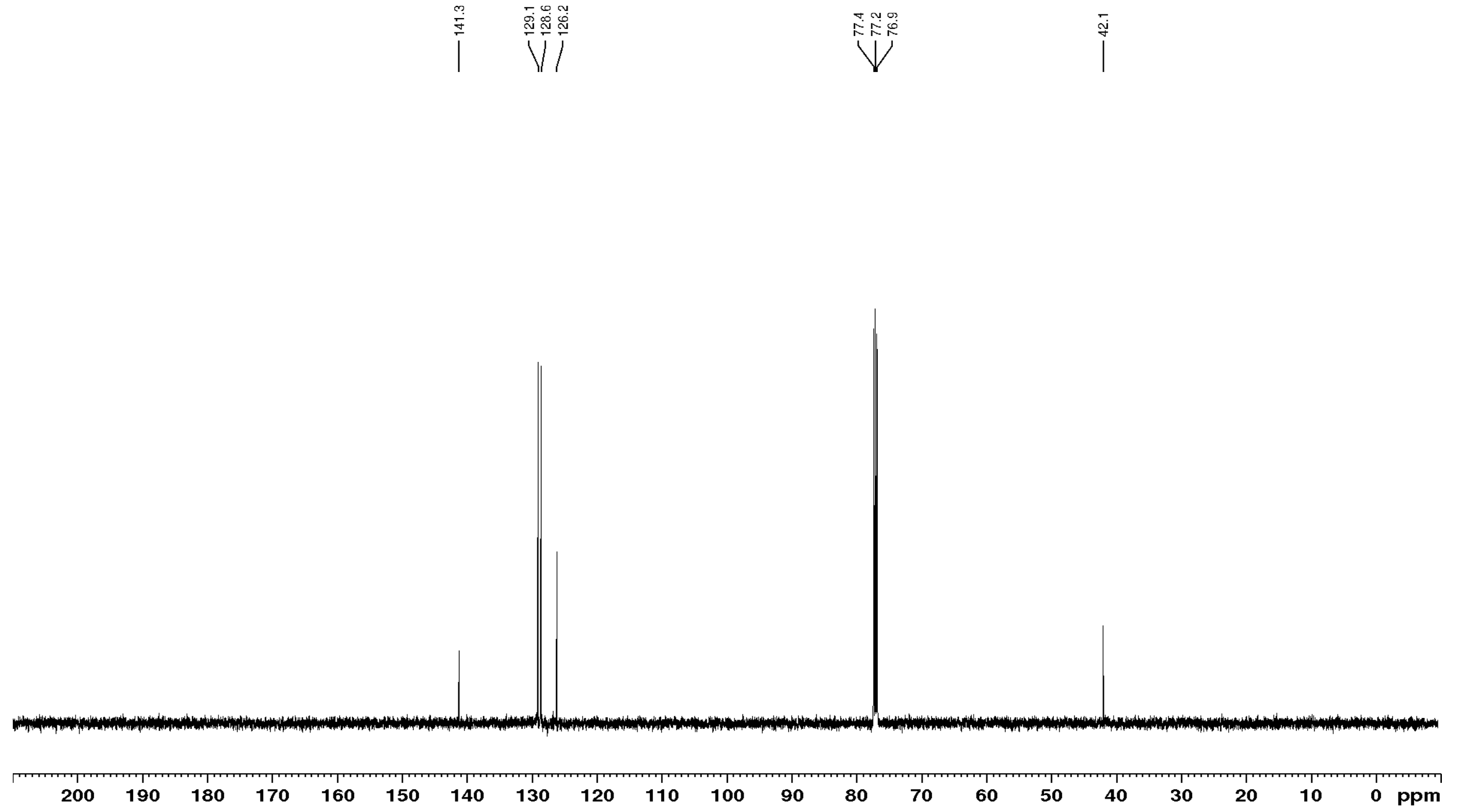
Figure S7. ${ }^{1} \mathbf{H}$ NMR spectrum ( $\left.500 \mathrm{MHz}, \mathrm{CDCl}_{3}, 298 \mathrm{~K}\right)$ of $\mathbf{3 f a}$ from the reaction of 1-(bromomethyl)-4-methylbenzene (1f) and benzene (2a).

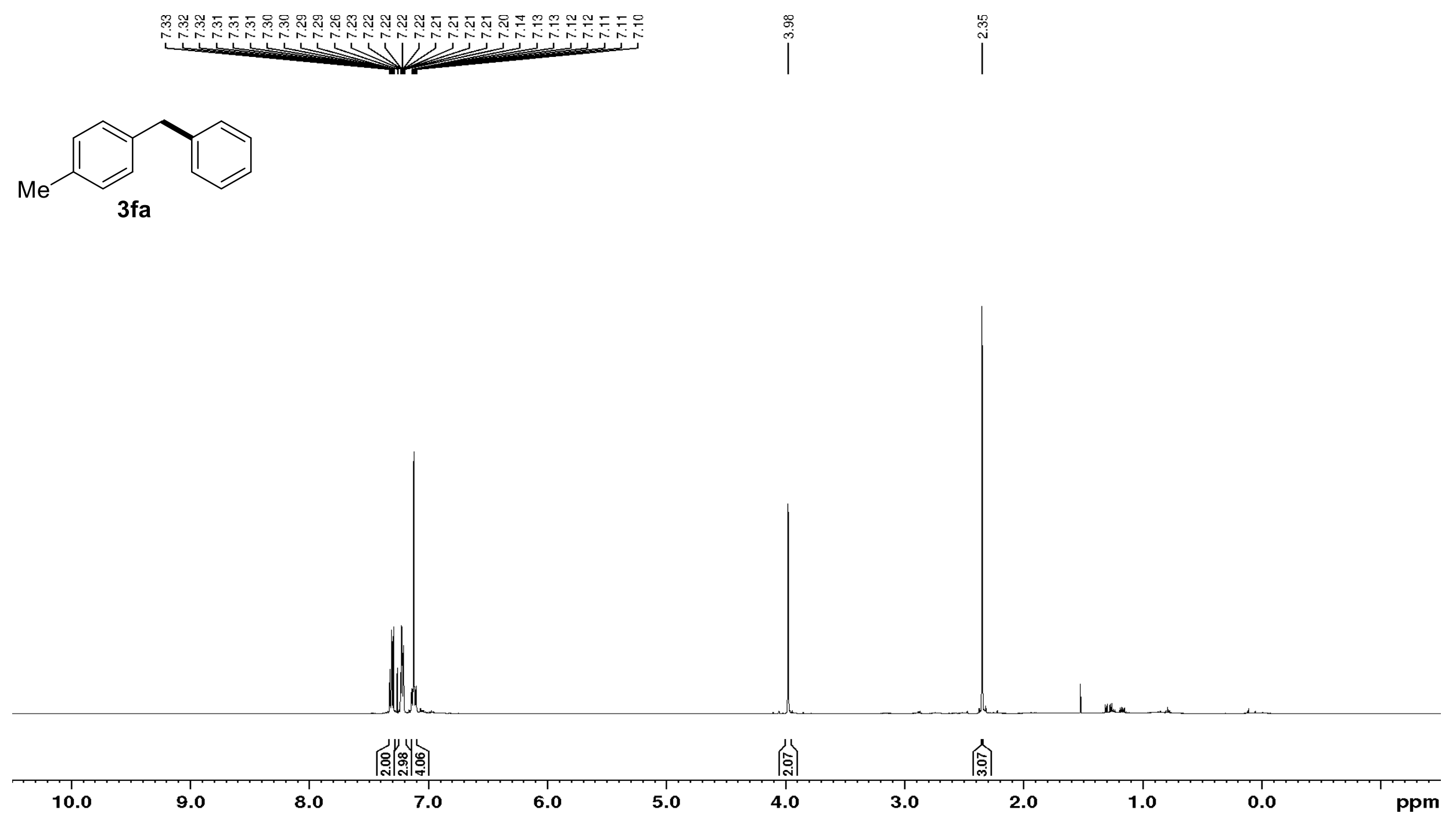


Figure S8. ${ }^{13} \mathrm{C}\left\{{ }^{1} \mathrm{H}\right\}$ NMR spectrum $\left(126 \mathrm{MHz}, \mathrm{CDCl}_{3}, 298 \mathrm{~K}\right)$ of 3 fa from the reaction of 1-(bromomethyl)-4-methylbenzene (1f) and benzene (2a).
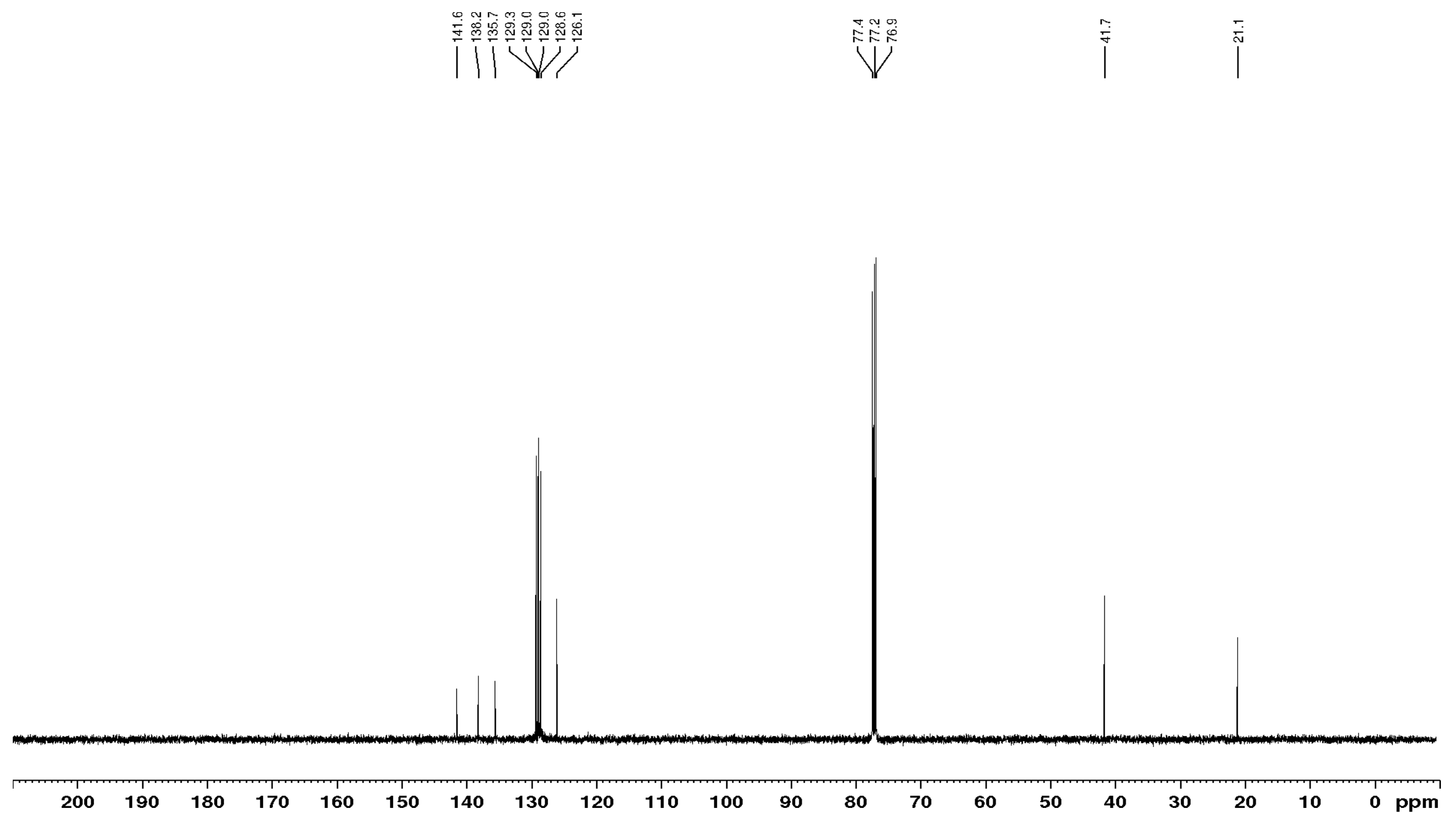
Figure S9. ${ }^{1} \mathbf{H}$ NMR spectrum $\left(500 \mathrm{MHz}, \mathrm{CDCl}_{3}, 298 \mathrm{~K}\right)$ of $\mathbf{3 g a}$ from the reaction of 1-(bromomethyl)-3-methylbenzene (19) and benzene (2a).

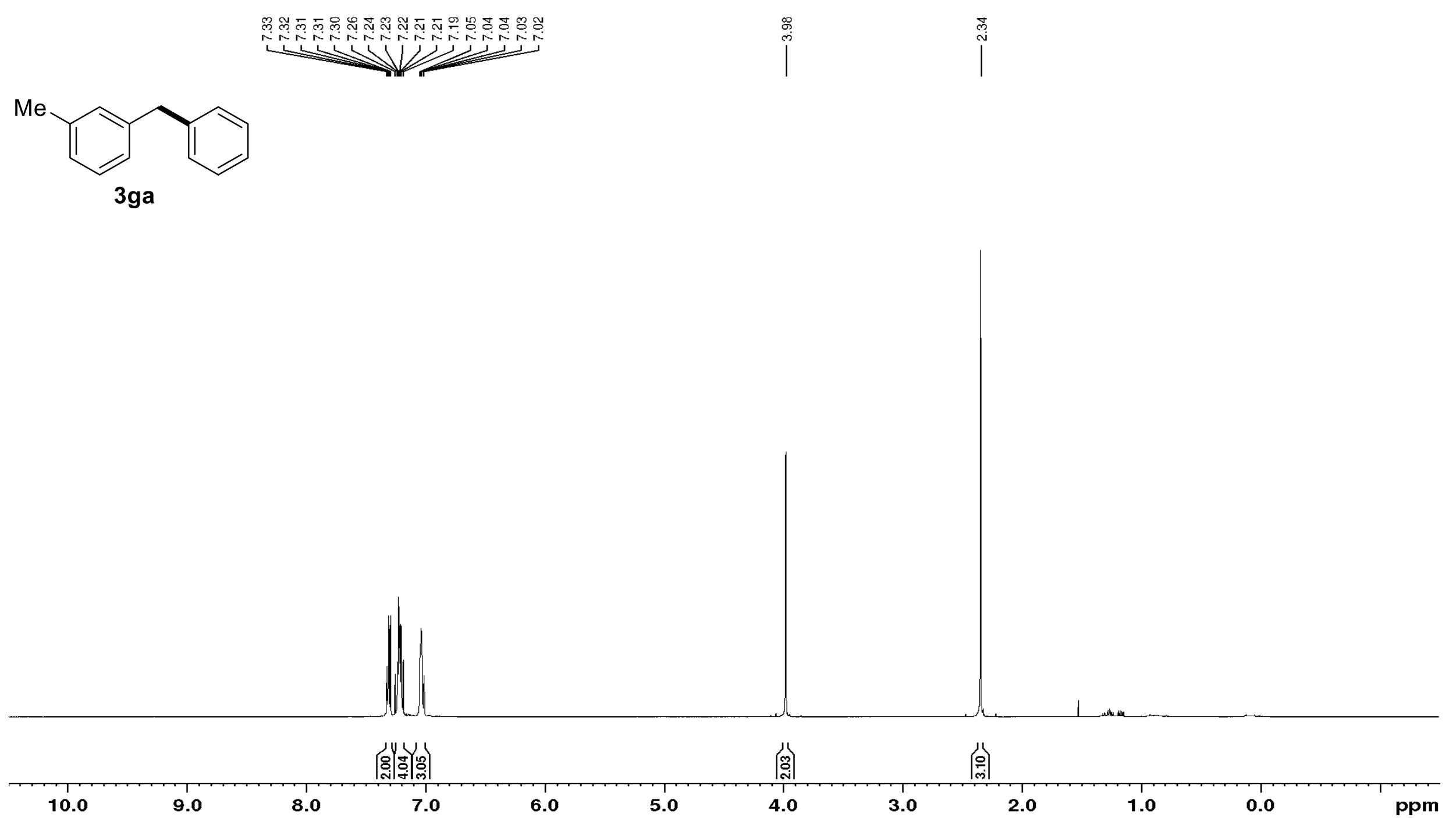


Figure S10. ${ }^{13} \mathbf{C}\left\{{ }^{1} \mathrm{H}\right\}$ NMR spectrum (126 MHz, CDCl $\left.3,298 \mathrm{~K}\right)$ of $\mathbf{3 g a}$ from the reaction of 1-(bromomethyl)-3-methylbenzene (19) and benzene (2a).

Figure S11. ${ }^{1} \mathrm{H}$ NMR spectrum ( $\left.500 \mathrm{MHz}, \mathrm{CDCl}_{3}, 298 \mathrm{~K}\right)$ of 3ha from the reaction of 1-(bromomethyl)-2-methylbenzene (1h) and benzene (2a).

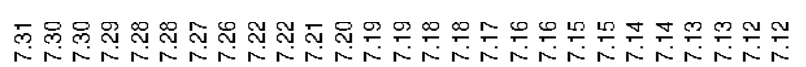



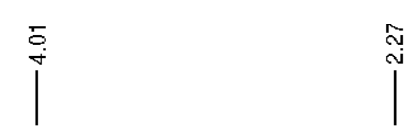

(N)

3ha




Figure S12. ${ }^{13} \mathbf{C}\left\{{ }^{1} \mathbf{H}\right\}$ NMR spectrum $\left(126 \mathrm{MHz}, \mathrm{CDCl}_{3}, 298 \mathrm{~K}\right)$ of 3 ha from the reaction of 1-(bromomethyl)-2-methylbenzene (1h) and benzene (2a).
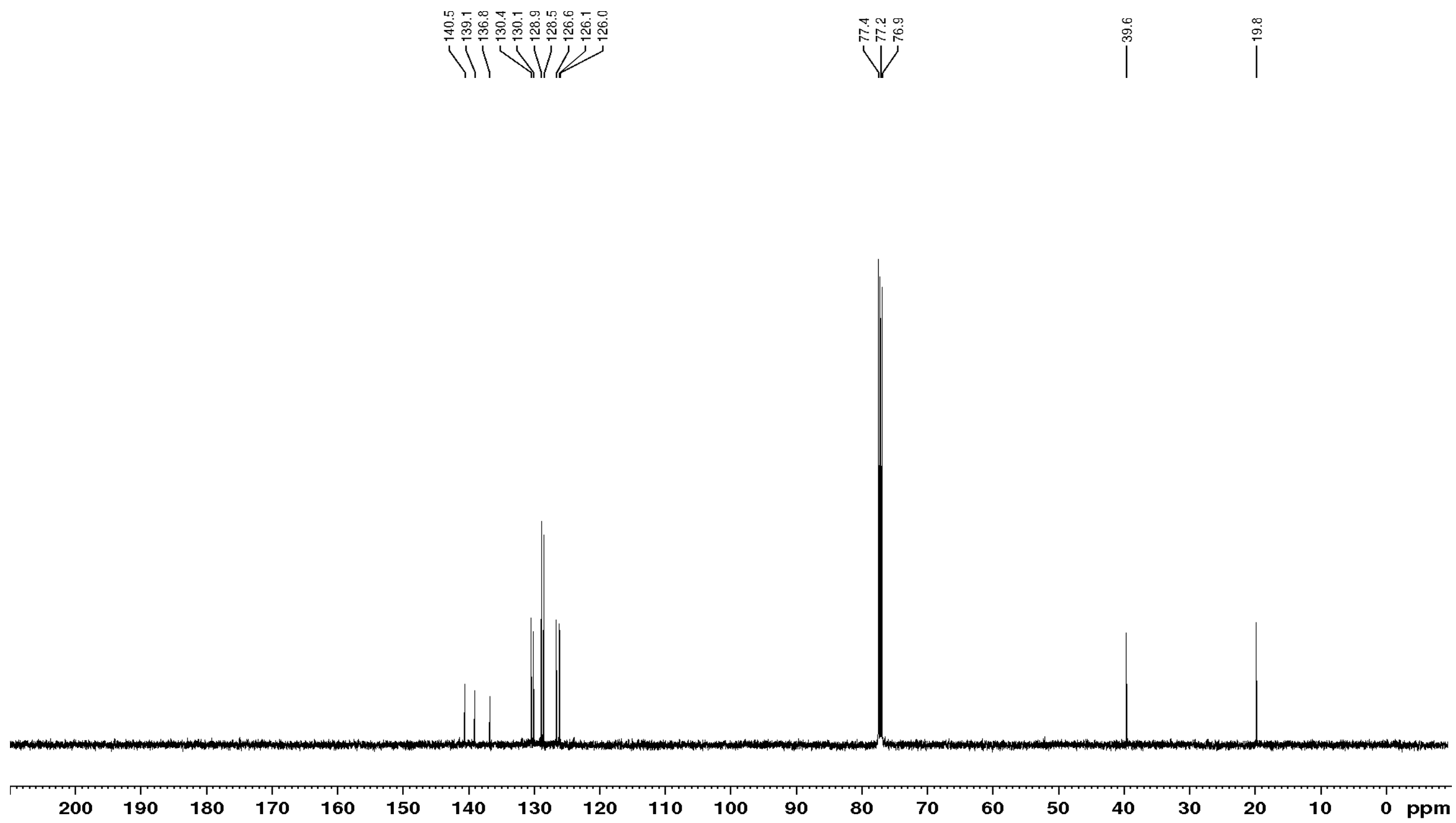
Figure S13. ${ }^{1} \mathrm{H}$ NMR spectrum $\left(500 \mathrm{MHz}, \mathrm{CDCl}_{3}, 298 \mathrm{~K}\right)$ of 3ia from the reaction of 1-(bromomethyl)-4-fluorobenze (1i) and benzene (2a).

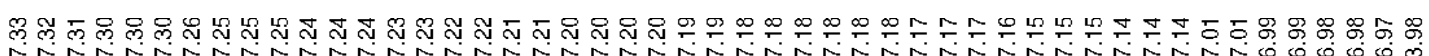



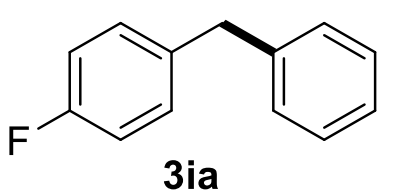

3ia




Figure S14. ${ }^{13} \mathbf{C}\left\{{ }^{1} \mathrm{H}\right\}$ NMR spectrum (126 MHz, $\left.\mathrm{CDCl}_{3}, 298 \mathrm{~K}\right)$ of 3ia from the reaction of 1-(bromomethyl)-4-fluorobenze (1i) and benzene (2a).
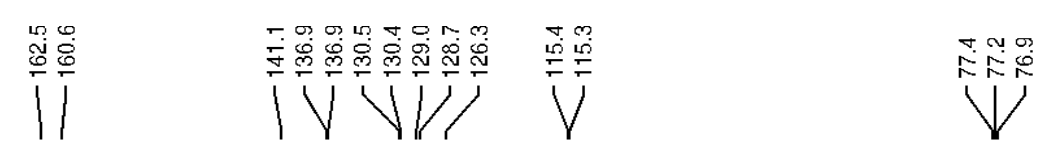

$\stackrel{\stackrel{\sim}{+}}{\tilde{F}}$




Figure S15. ${ }^{19} \mathrm{~F}$ NMR spectrum $\left(471 \mathrm{MHz}, \mathrm{CDCl}_{3}, 298 \mathrm{~K}\right)$ of 3ia the reaction of 1-(bromomethyl)-4-fluorobenze (1i) and benzene (2a).

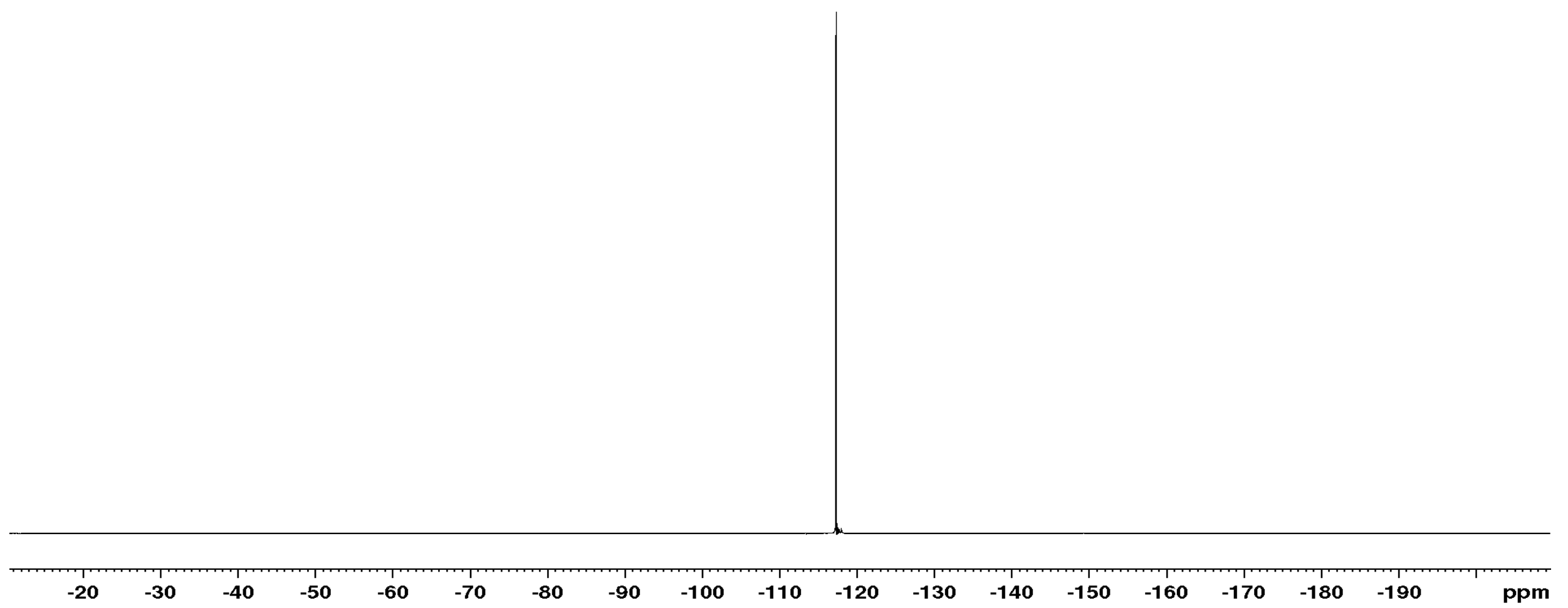


Figure S16. ${ }^{1} \mathrm{H}$ NMR spectrum $\left(500 \mathrm{MHz}, \mathrm{CDCl}_{3}, 298 \mathrm{~K}\right)$ of 3ja from the reaction of 1-(bromomethyl)-3-fluorobenzene (1j) and benzene (2a).

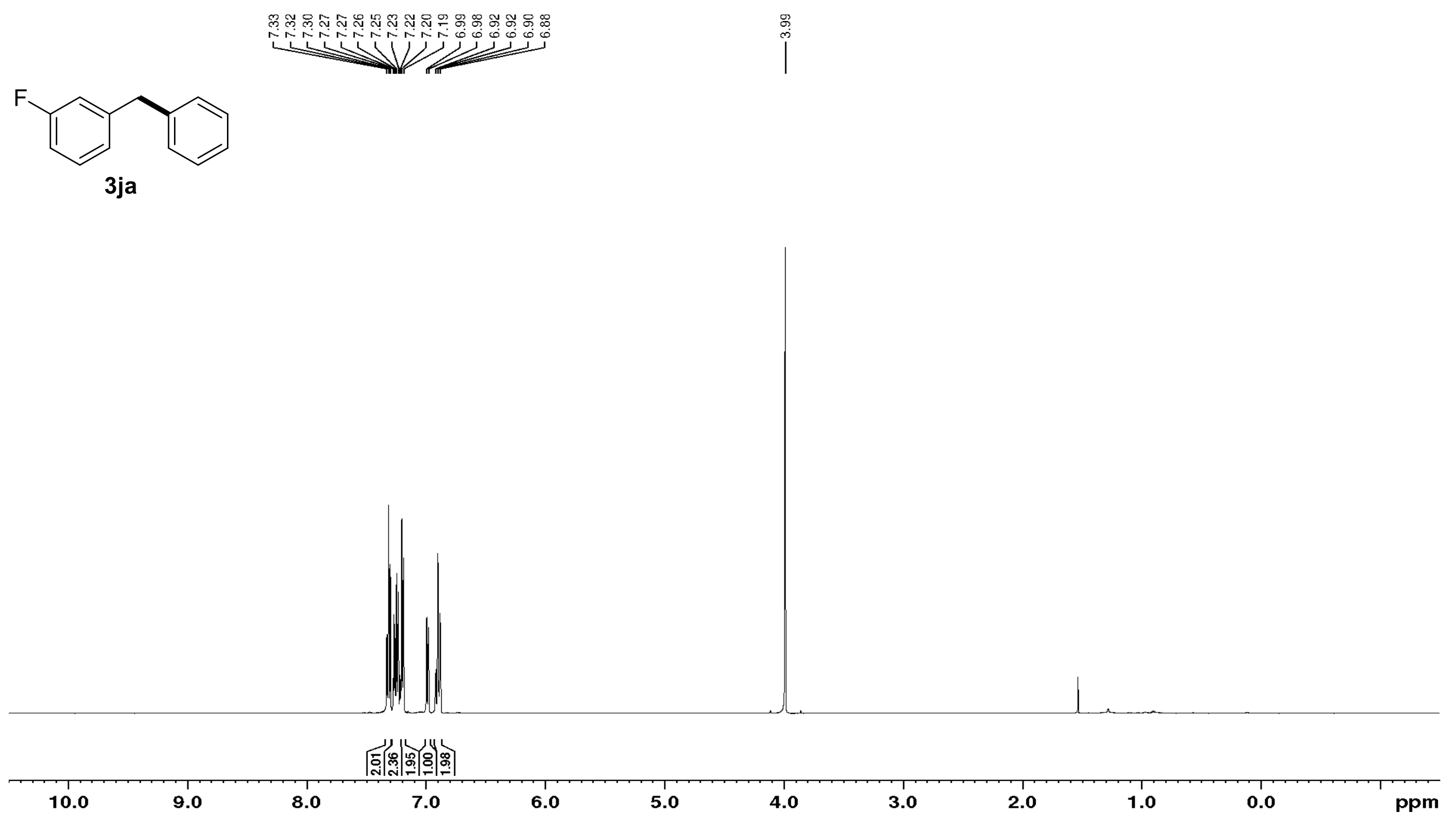


Figure S17. ${ }^{13} \mathrm{C}\left\{{ }^{1} \mathbf{H}\right\}$ NMR spectrum $\left(126 \mathrm{MHz}, \mathrm{CDCl}_{3}, 298 \mathrm{~K}\right)$ of 3ja from the reaction of 1-(bromomethyl)-3-fluorobenzene (1j) and benzene (2a).

Figure S18. ${ }^{19} \mathrm{~F}$ NMR spectrum $\left(471 \mathrm{MHz}, \mathrm{CDCl}_{3}, 298 \mathrm{~K}\right)$ of $\mathbf{3 j a}$ from from the reaction of 1-(bromomethyl)-3-fluorobenzene (1j) and benzene (2a).

$$
\stackrel{\stackrel{0}{i}}{i}
$$

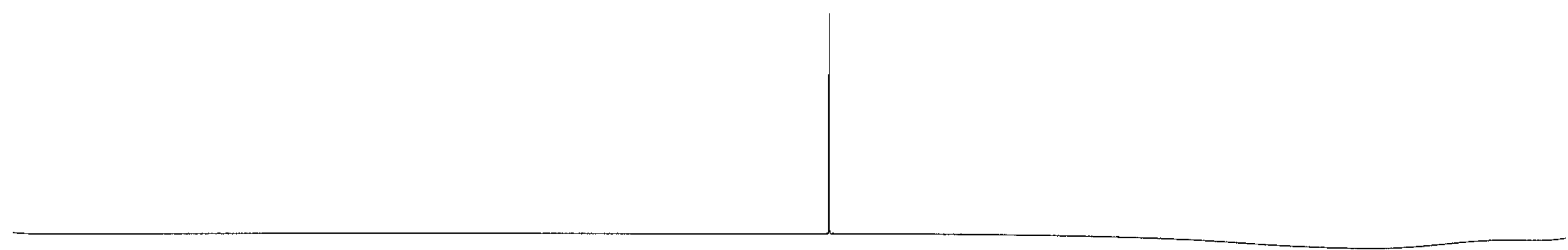

\begin{tabular}{|c|c|c|c|c|c|c|c|c|c|c|c|c|}
\hline-50 & -60 & -70 & -80 & -90 & -100 & -110 & -120 & -130 & -140 & -150 & -160 & -170 \\
\hline
\end{tabular}


Figure S19. ${ }^{1} \mathbf{H}$ NMR spectrum $\left(500 \mathrm{MHz}, \mathrm{CDCl}_{3}, 298 \mathrm{~K}\right)$ of $\mathbf{3 k a}$ from the reaction of 1-(bromomethyl)-2-fluorobenzene (1k) and benzene (2a).
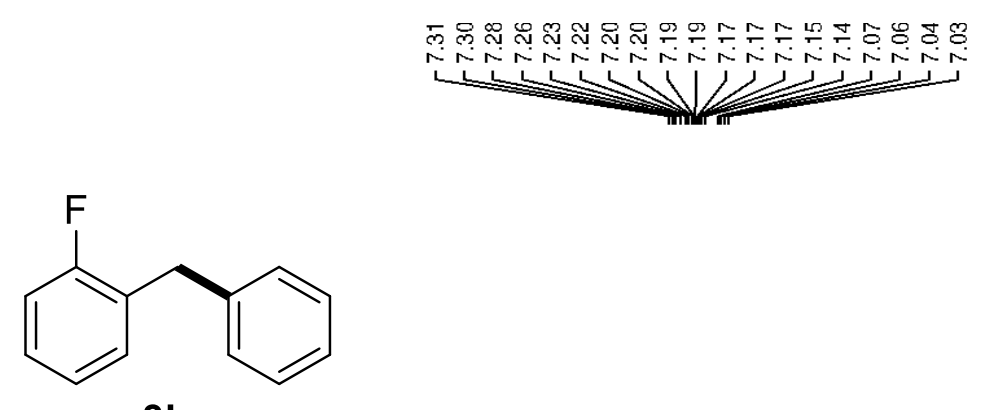

3ka

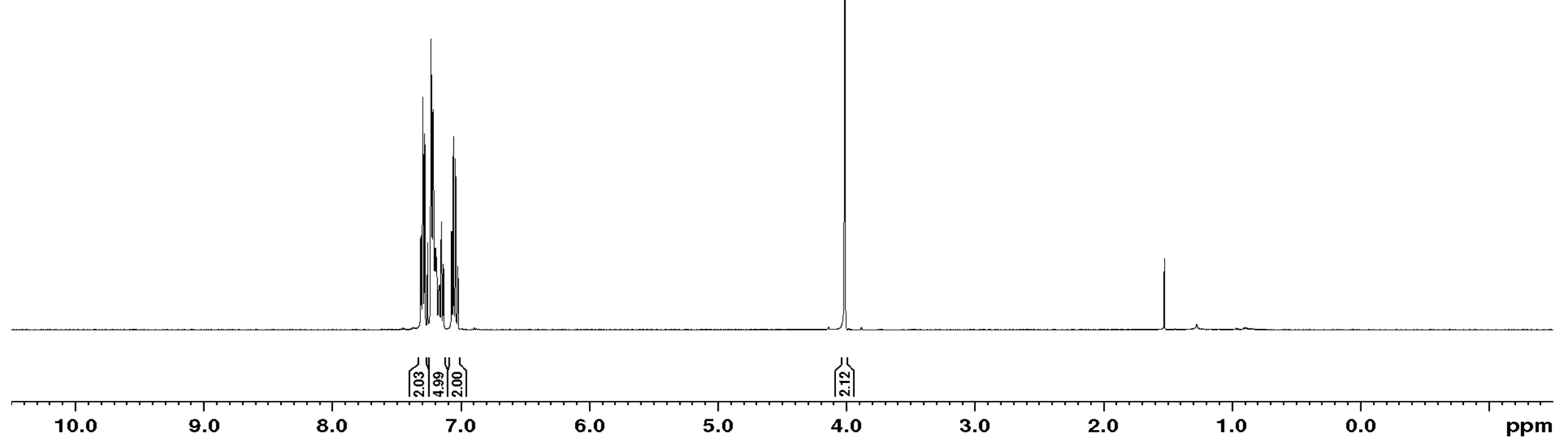


Figure S20. ${ }^{13} \mathbf{C}\left\{{ }^{1} \mathbf{H}\right\}$ NMR spectrum (126 MHz, CDCl $\left.3,298 \mathrm{~K}\right)$ of 3ka from the reaction of 1-(bromomethyl)-2-fluorobenzene (1k) and benzene (2a).
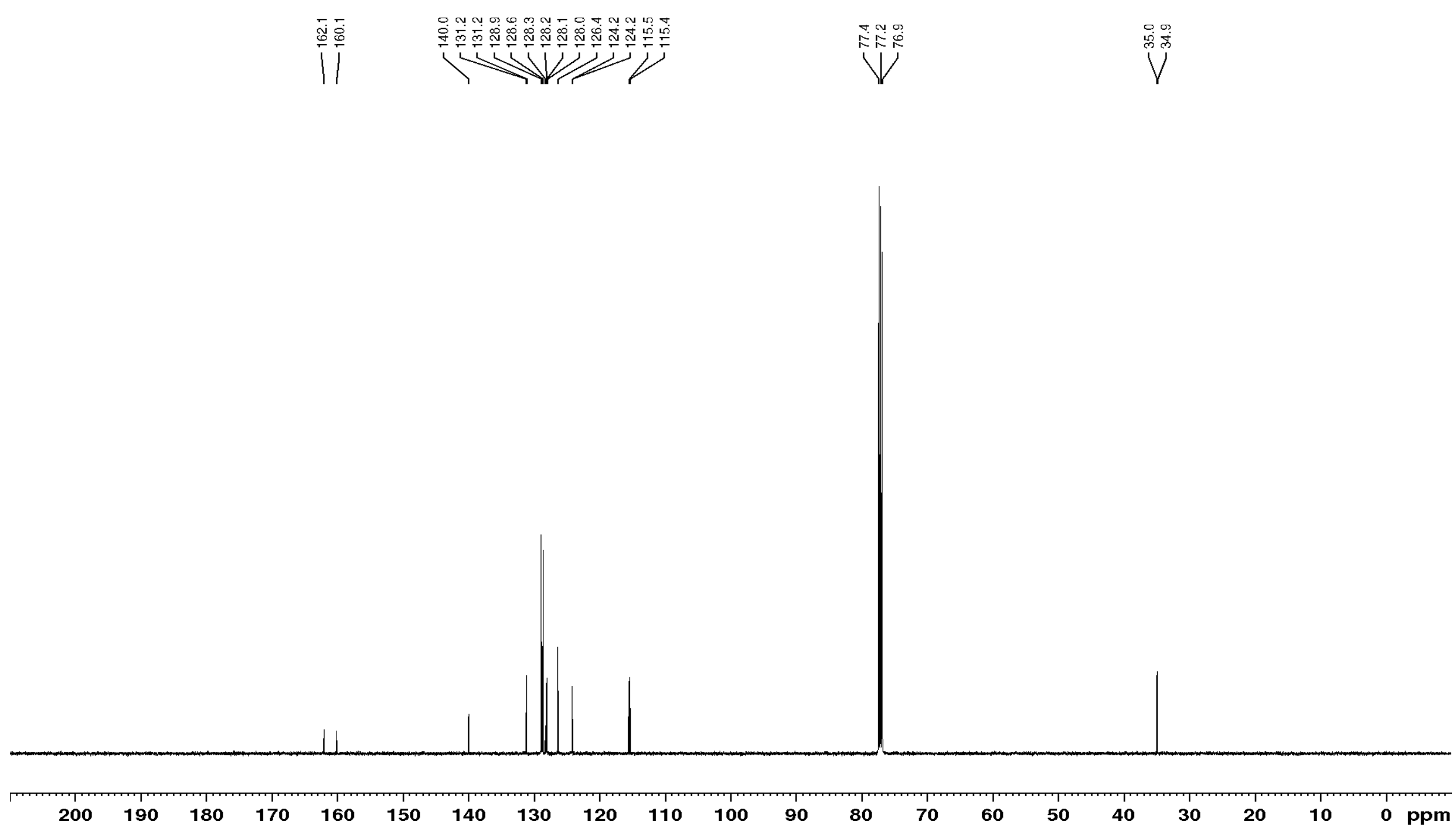
Figure S21. ${ }^{19} \mathrm{~F} \mathrm{NMR}$ spectrum $\left(471 \mathrm{MHz}, \mathrm{CDCl}_{3}, 298 \mathrm{~K}\right)$ of $\mathbf{3 k a}$ from the reaction of 1-(bromomethyl)-2-fluorobenzene (1k) and benzene (2a).

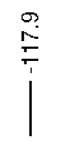

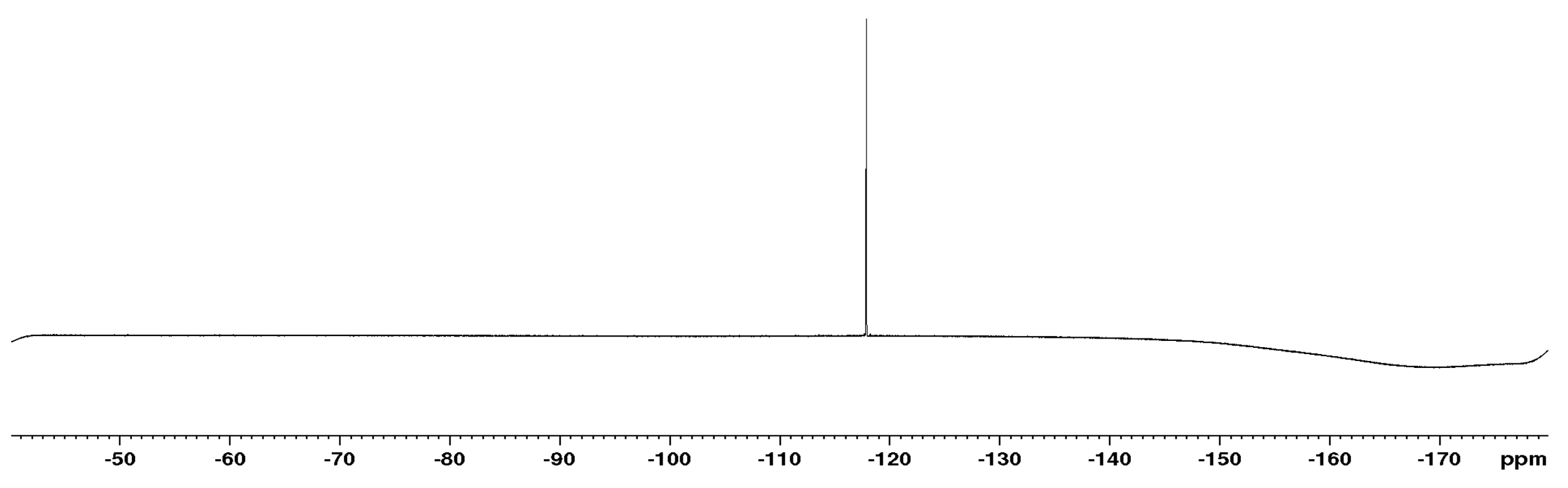


Figure S22. ${ }^{1} \mathrm{H}$ NMR spectrum $\left(500 \mathrm{MHz}, \mathrm{CDCl}_{3}, 298 \mathrm{~K}\right.$ ) of 3la from the reaction of 1-(bromomethyl)-4-chlorobenzene (1) and benzene (2a).
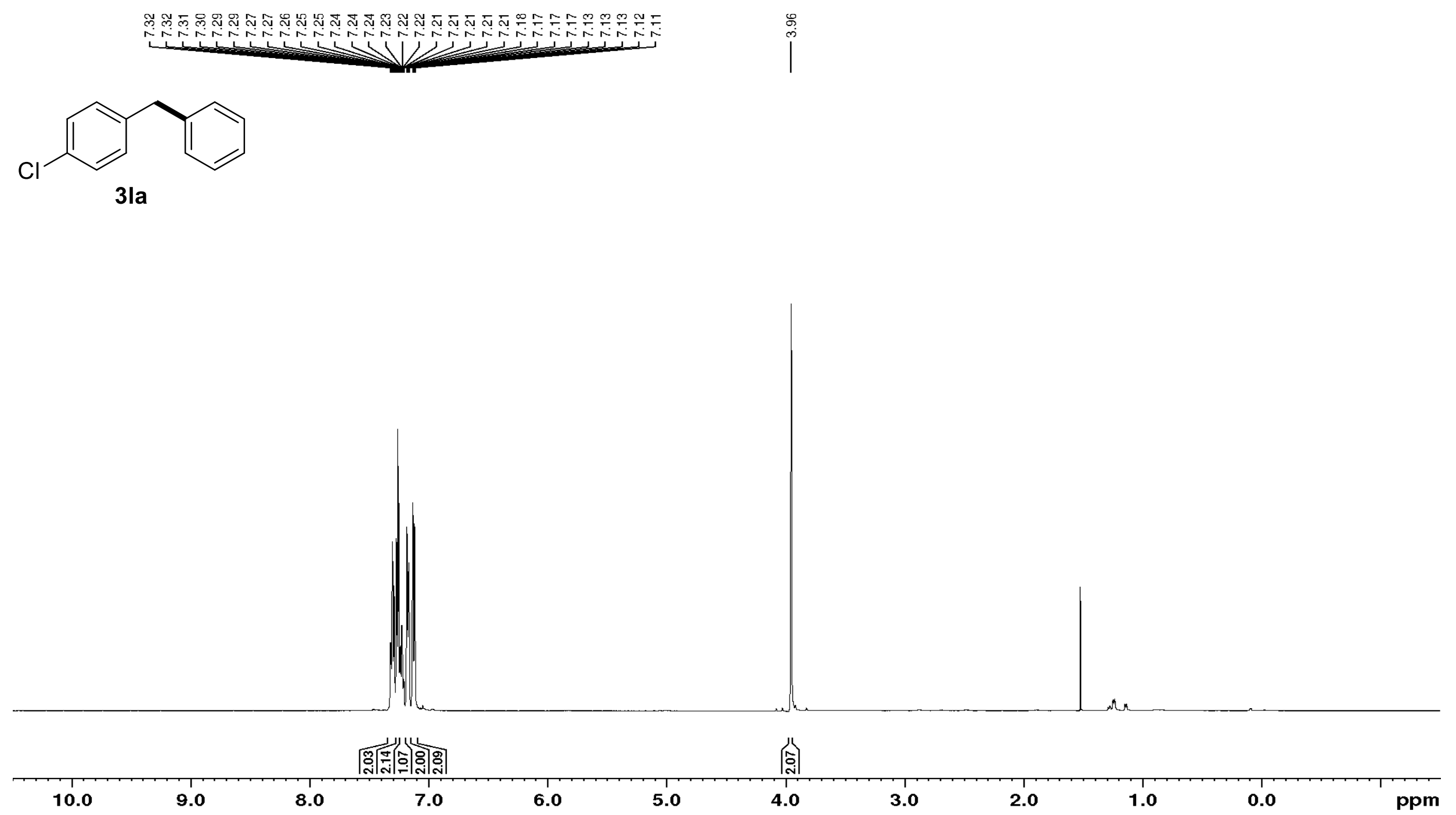
Figure S23. ${ }^{13} \mathrm{C}\left\{{ }^{1} \mathrm{H}\right\}$ NMR spectrum (126 MHz, $\left.\mathrm{CDCl}_{3}, 298 \mathrm{~K}\right)$ of 3la from the reaction of 1-(bromomethyl)-4-chlorobenzene (11) and benzene (2a).
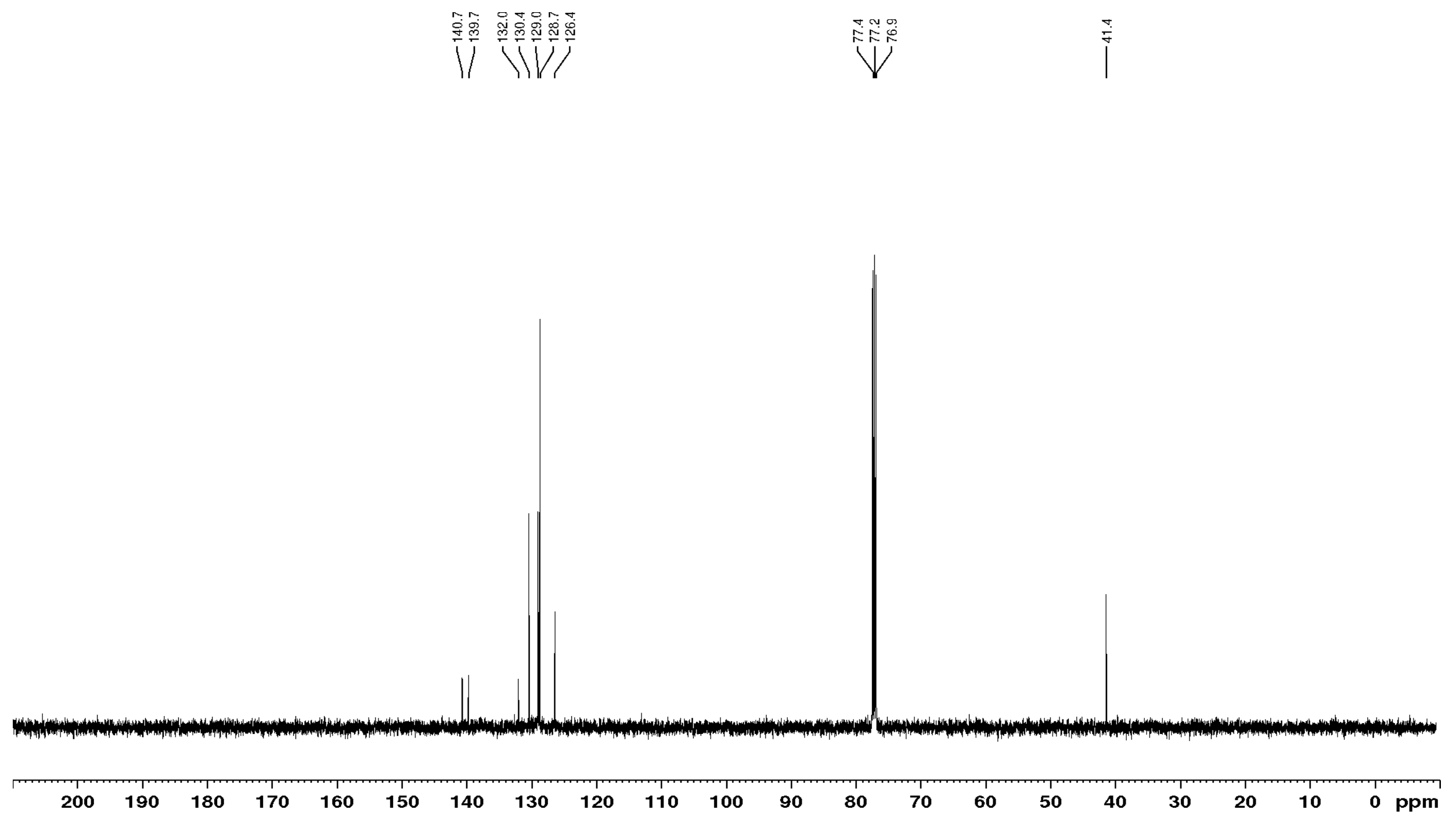
Figure S24. ${ }^{1} \mathrm{H}$ NMR spectrum $\left(500 \mathrm{MHz}, \mathrm{CDCl}_{3}, 298 \mathrm{~K}\right)$ of $3 \mathrm{ma}$ from the reaction of 1-(bromomethyl)-3-chlorobenzene (1 $\left.\mathbf{m}\right)$ and benzene (2a).





Cl

$3 \mathrm{ma}$




Figure S25. ${ }^{13} \mathrm{C}\left\{{ }^{1} \mathrm{H}\right\}$ NMR spectrum $\left(126 \mathrm{MHz}, \mathrm{CDCl}_{3}, 298 \mathrm{~K}\right)$ of $3 \mathrm{ma}$ from the reaction of 1-(bromomethyl)-3-chlorobenzene (1m) and benzene $(\mathbf{2 a})$.
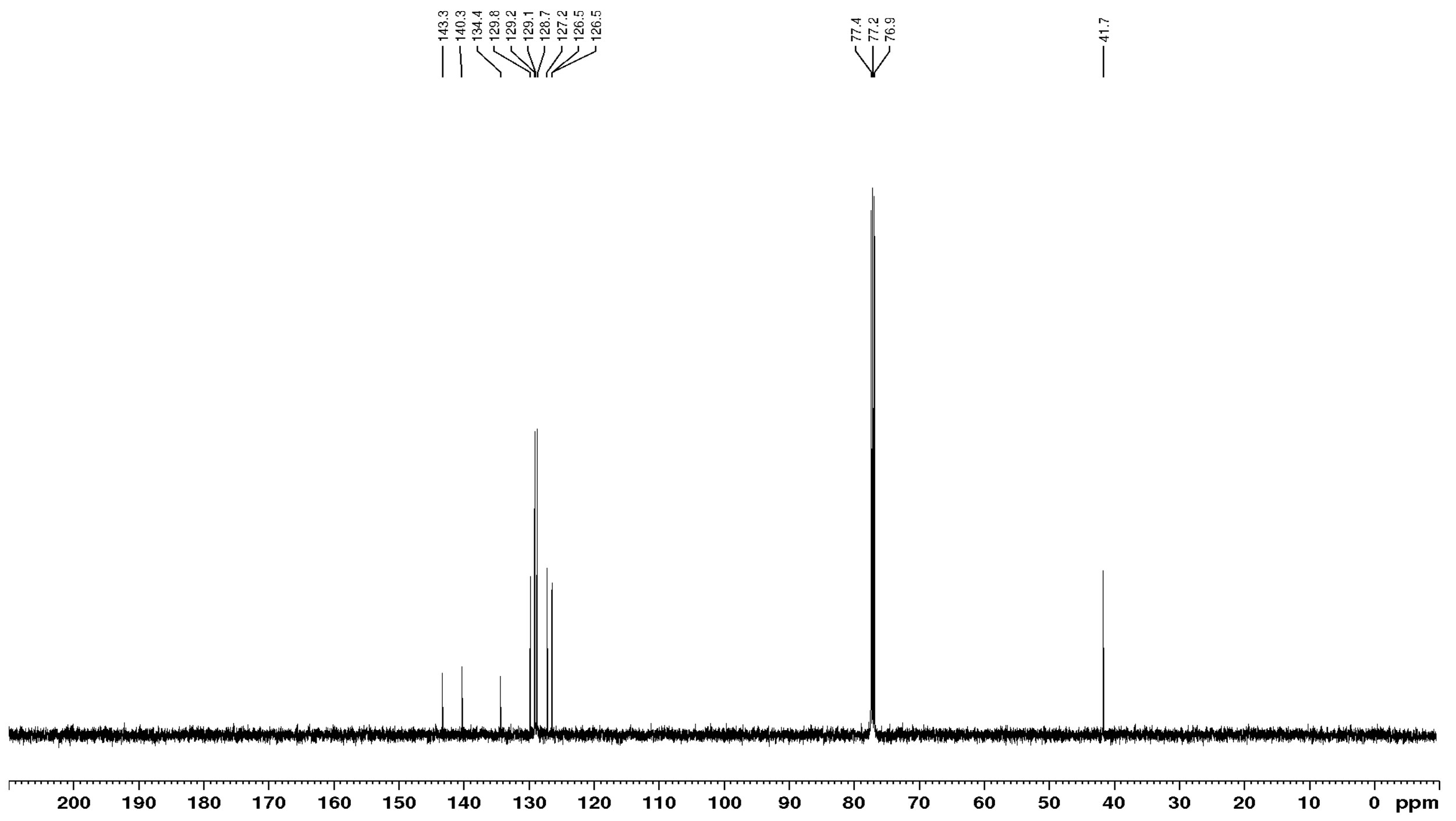
Figure S26. ${ }^{1} \mathbf{H}$ NMR spectrum ( $\left.500 \mathrm{MHz}, \mathrm{CDCl}_{3}, 298 \mathrm{~K}\right)$ of 3na from the reaction of 1-(bromomethyl)-2-chlorobenzene (1n) and benzene (2a).
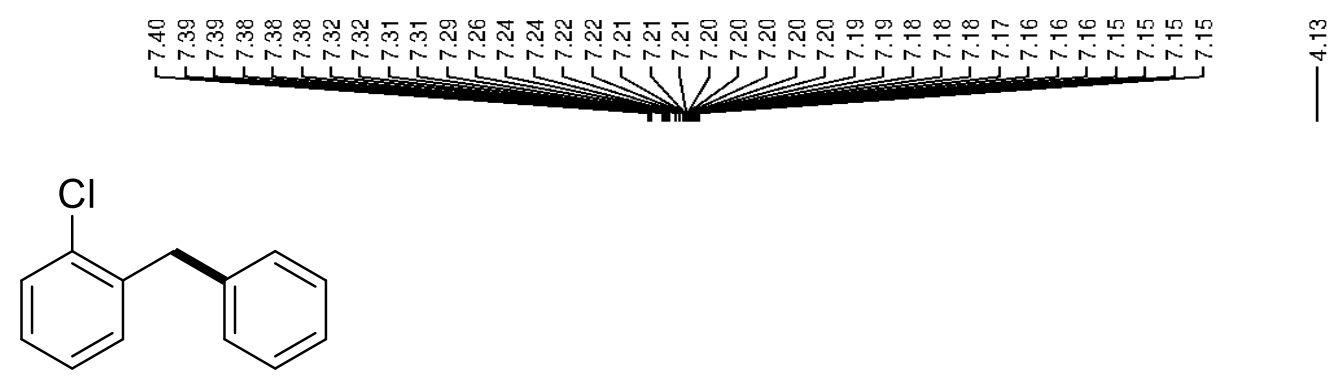

3na




Figure S27. ${ }^{13} \mathbf{C}\left\{{ }^{1} \mathrm{H}\right\}$ NMR spectrum (126 MHz, $\left.\mathrm{CDCl}_{3}, 298 \mathrm{~K}\right)$ of 3na from the reaction of 1-(bromomethyl)-2-chlorobenzene (1n) and benzene (2a).

Figure S28. ${ }^{1} \mathrm{H}$ NMR spectrum ( $500 \mathrm{MHz}, \mathrm{CDCl}_{3}, 298 \mathrm{~K}$ ) of 3oa from the reaction of 1-bromo-4-(bromomethyl)benzene (10) and benzene (2a).
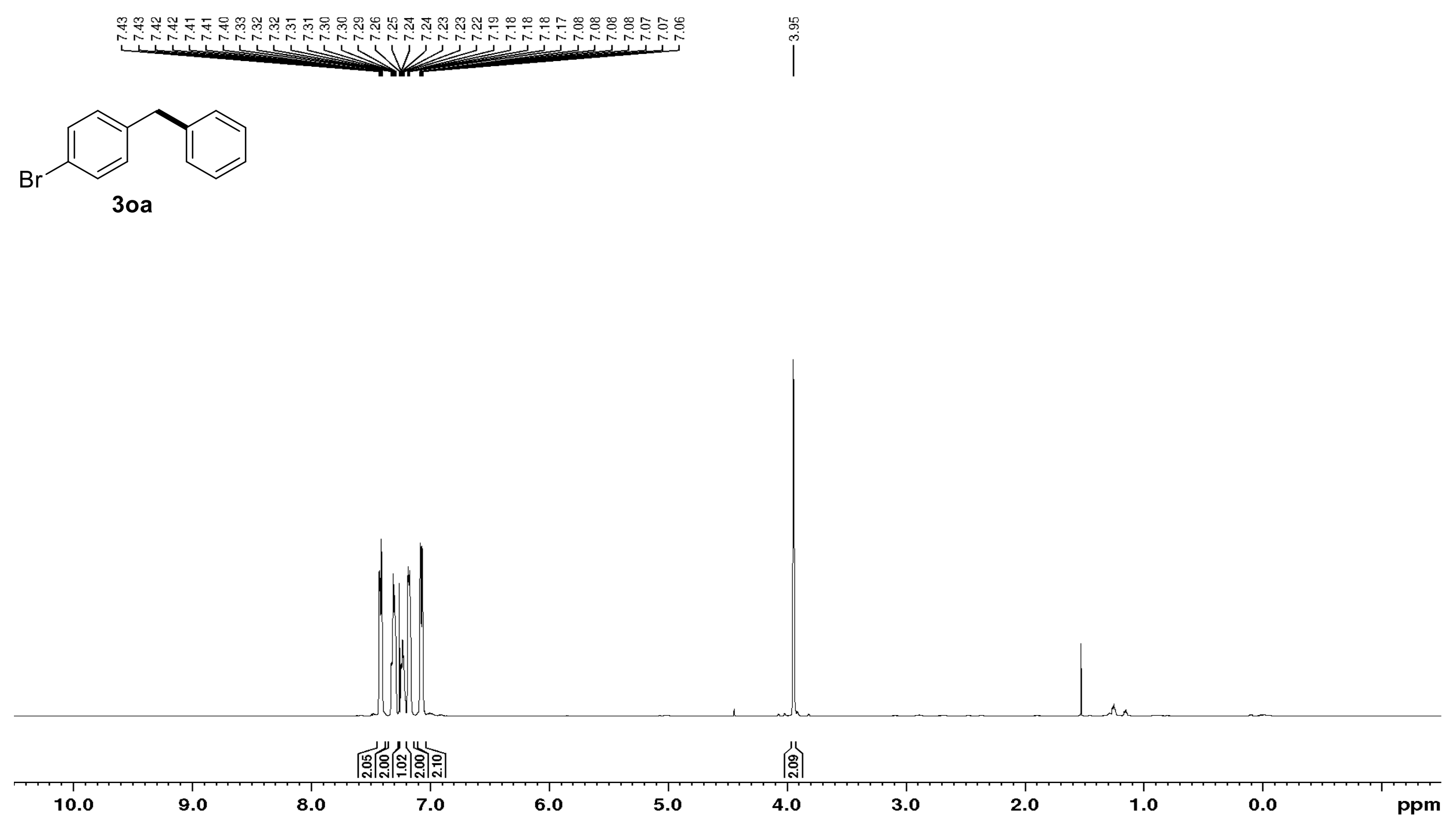
Figure S29. ${ }^{13} \mathbf{C}\left\{{ }^{1} \mathrm{H}\right\}$ NMR spectrum (126 MHz, $\left.\mathrm{CDCl}_{3}, 298 \mathrm{~K}\right)$ of $30 a$ from the reaction of 1-bromo-4-(bromomethyl)benzene (10) and benzene (2a).
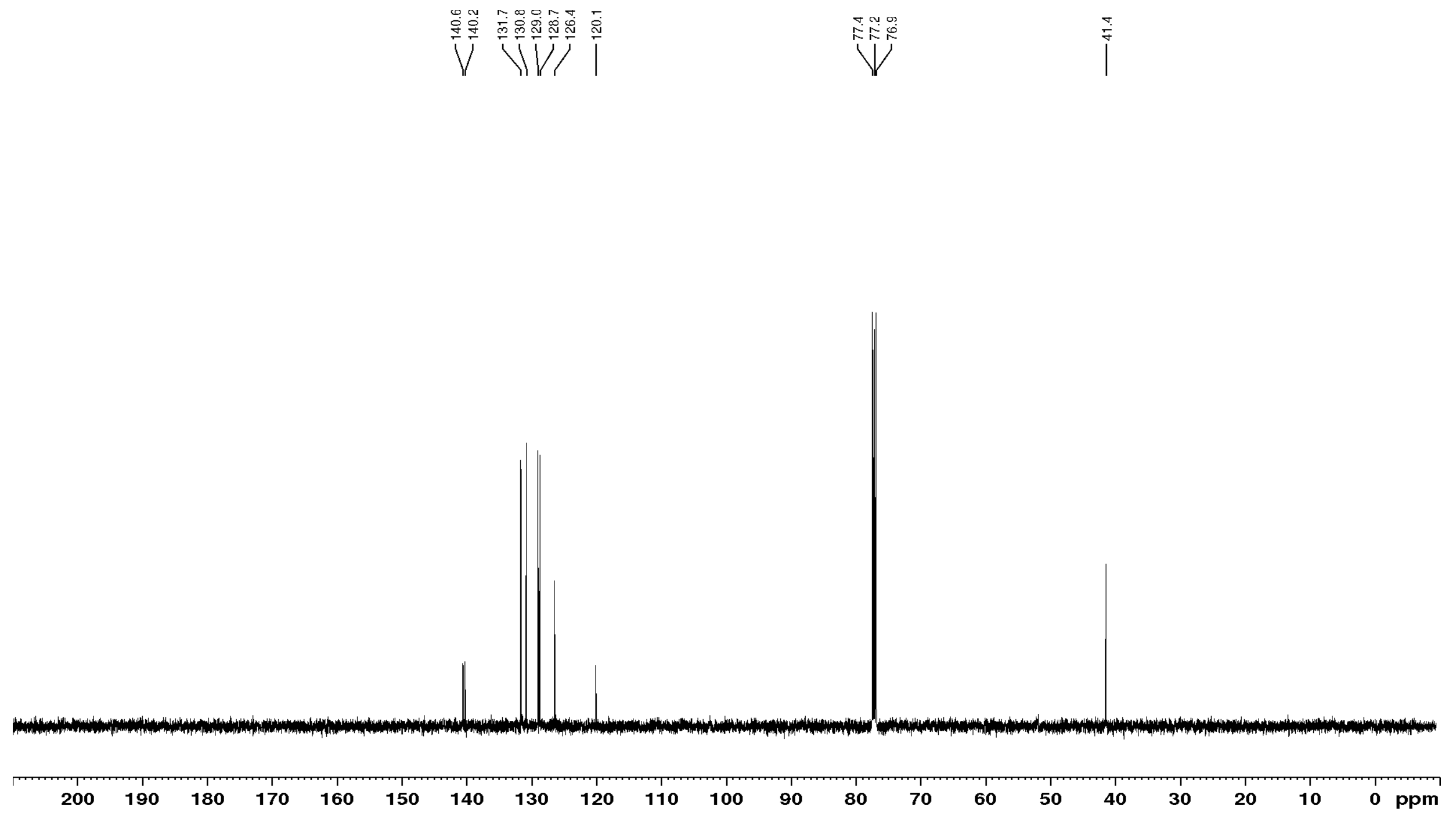
Figure S30. ${ }^{1} \mathrm{H}$ NMR spectrum (500 MHz, $\left.\mathrm{CDCl}_{3}, 298 \mathrm{~K}\right)$ of 3pa from the reaction of 1-bromo-3-(bromomethyl)benzene (1p) and benzene (2a).

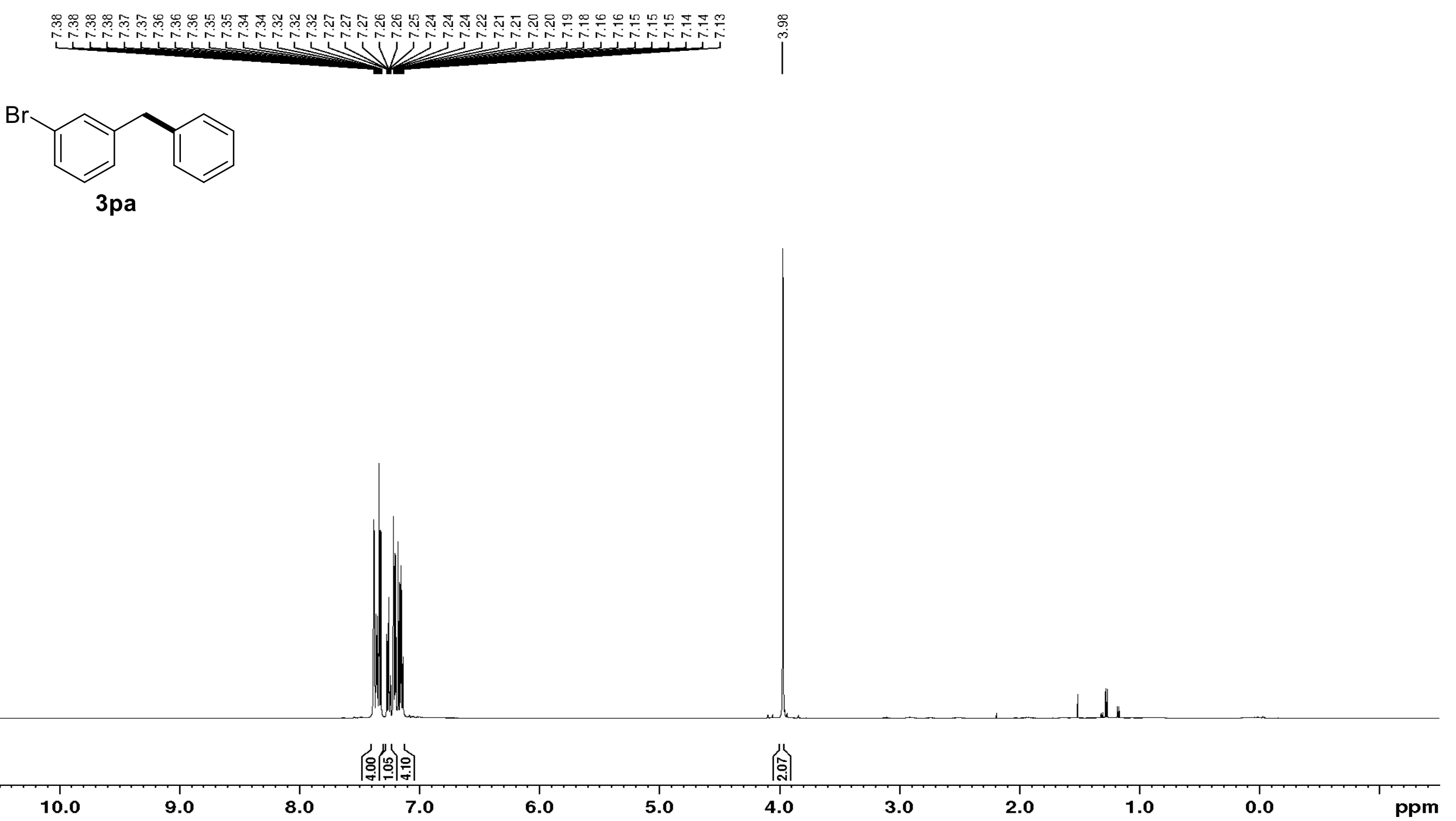


Figure S31. ${ }^{13} \mathbf{C}\left\{{ }^{1} \mathbf{H}\right\}$ NMR spectrum (126 MHz, CDCl $\left.3,298 \mathrm{~K}\right)$ of 3pa from the reaction of 1-bromo-3-(bromomethyl)benzene (1p) and benzene (2a).
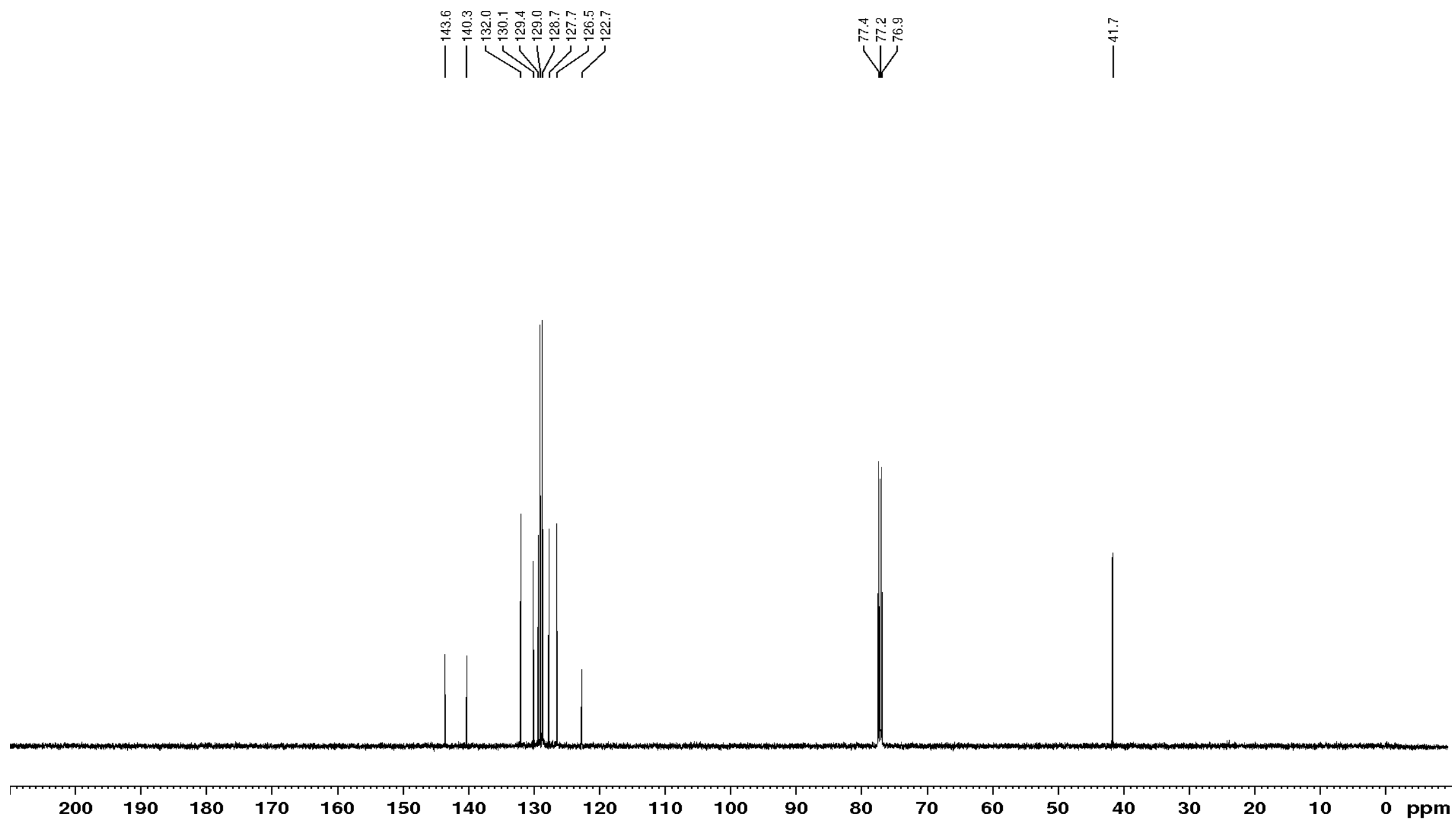
Figure S32. ${ }^{1} \mathbf{H}$ NMR spectrum $\left(500 \mathrm{MHz}, \mathrm{CDCl}_{3}, 298 \mathrm{~K}\right)$ of $\mathbf{3 q a}$ from the reaction of 1-bromo-2-(bromomethyl)benzene (1q) and benzene (2a).


$3 q a$




Figure S33. ${ }^{13} \mathbf{C}\left\{{ }^{1} \mathrm{H}\right\}$ NMR spectrum (126 MHz, $\left.\mathrm{CDCl}_{3}, 298 \mathrm{~K}\right)$ of 3qa from the reaction of 1-bromo-2-(bromomethyl)benzene (1q) and benzene (2a).
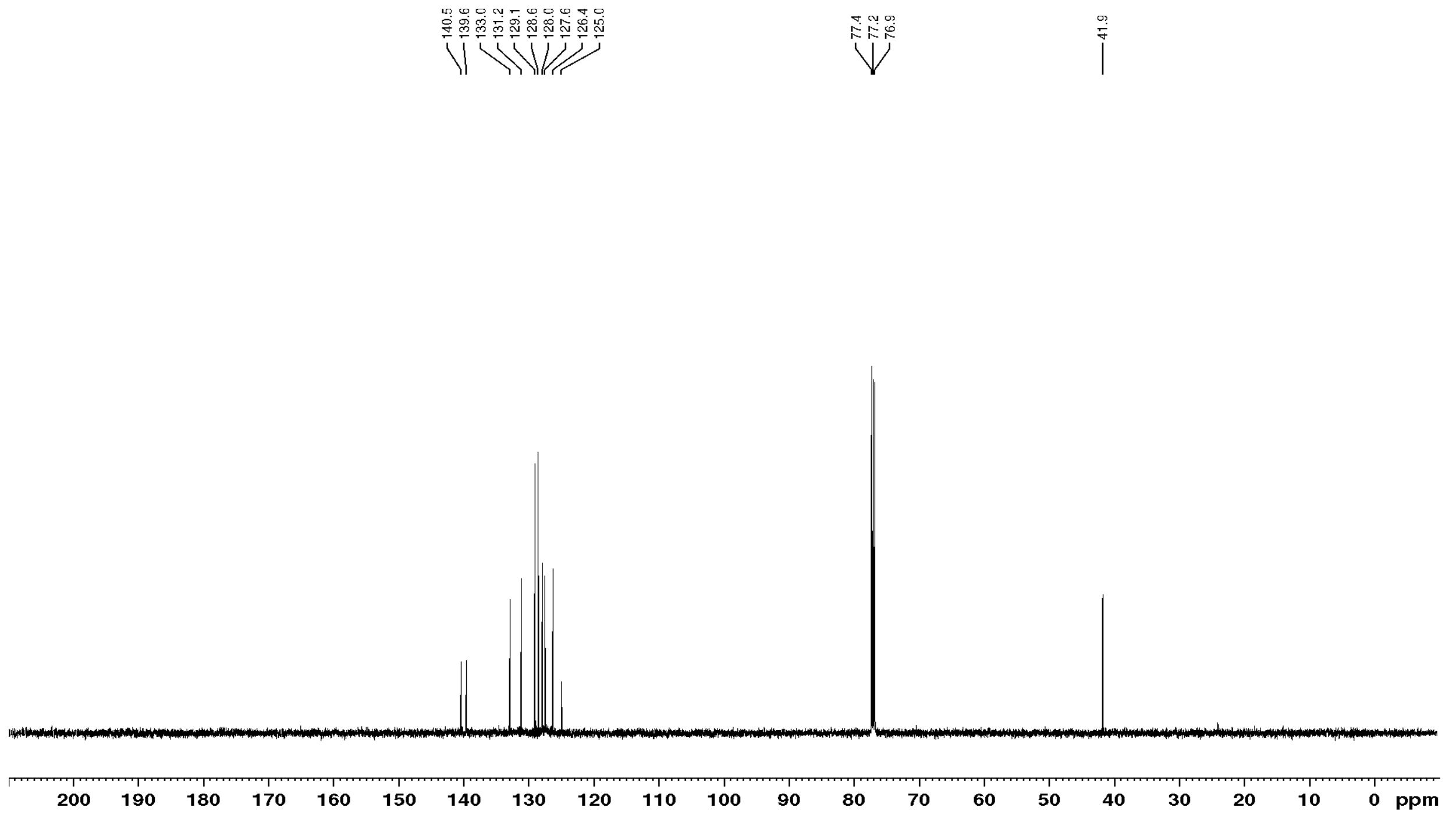
Figure S34. ${ }^{1} \mathrm{H}$ NMR spectrum $\left(400 \mathrm{MHz}, \mathrm{CDCl}_{3}, 298 \mathrm{~K}\right)$ of 3ra from the reaction of 1-(bromomethyl)-4-iodobenzene (1r) and benzene (2a).

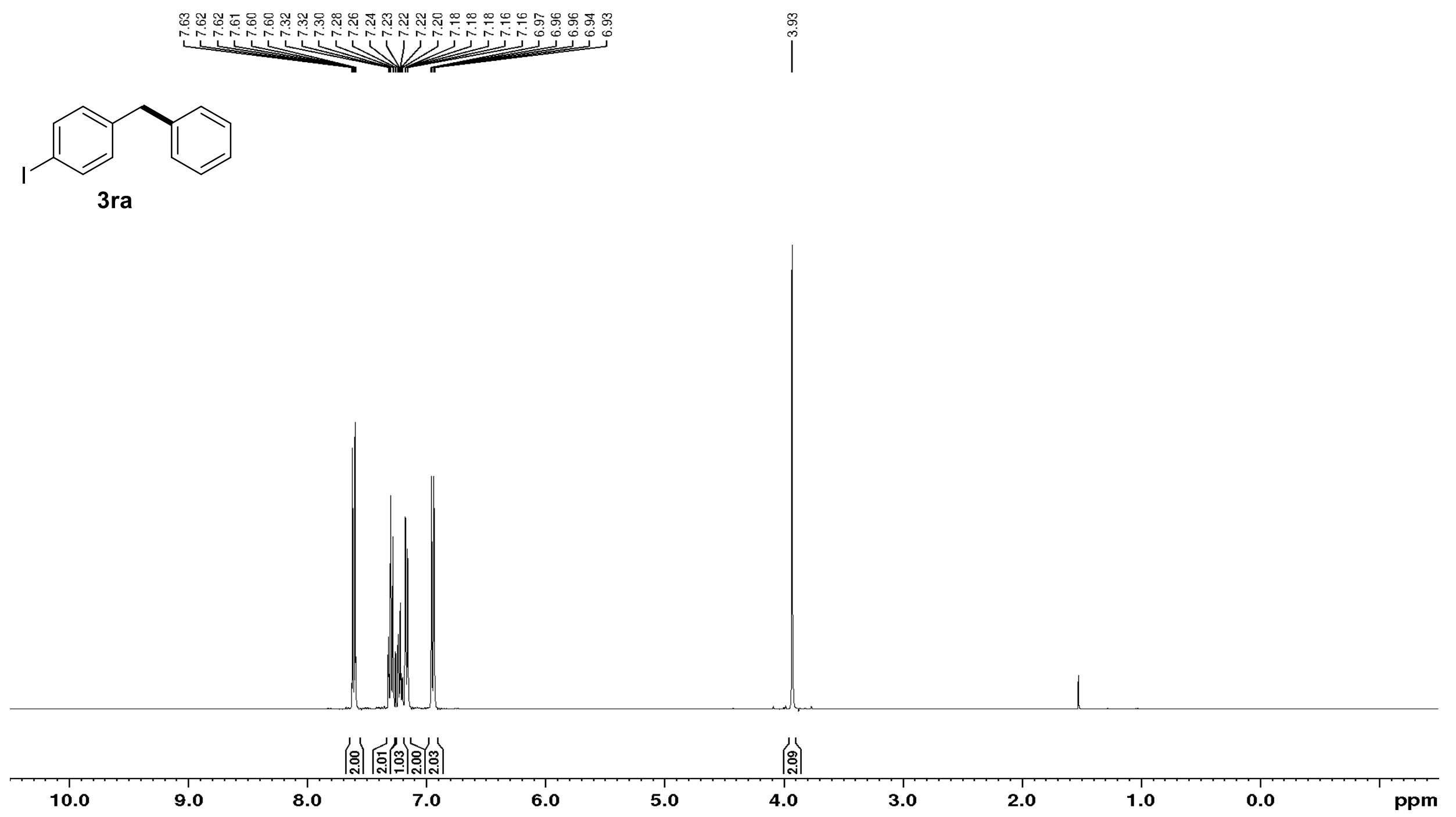


Figure S35. ${ }^{13} \mathrm{C}\left\{{ }^{1} \mathrm{H}\right\}$ NMR spectrum $\left(101 \mathrm{MHz}, \mathrm{CDCl}_{3}, 298 \mathrm{~K}\right)$ of 3ra from the reaction of 1-(bromomethyl)-4-iodobenzene (1r) and benzene (2a).
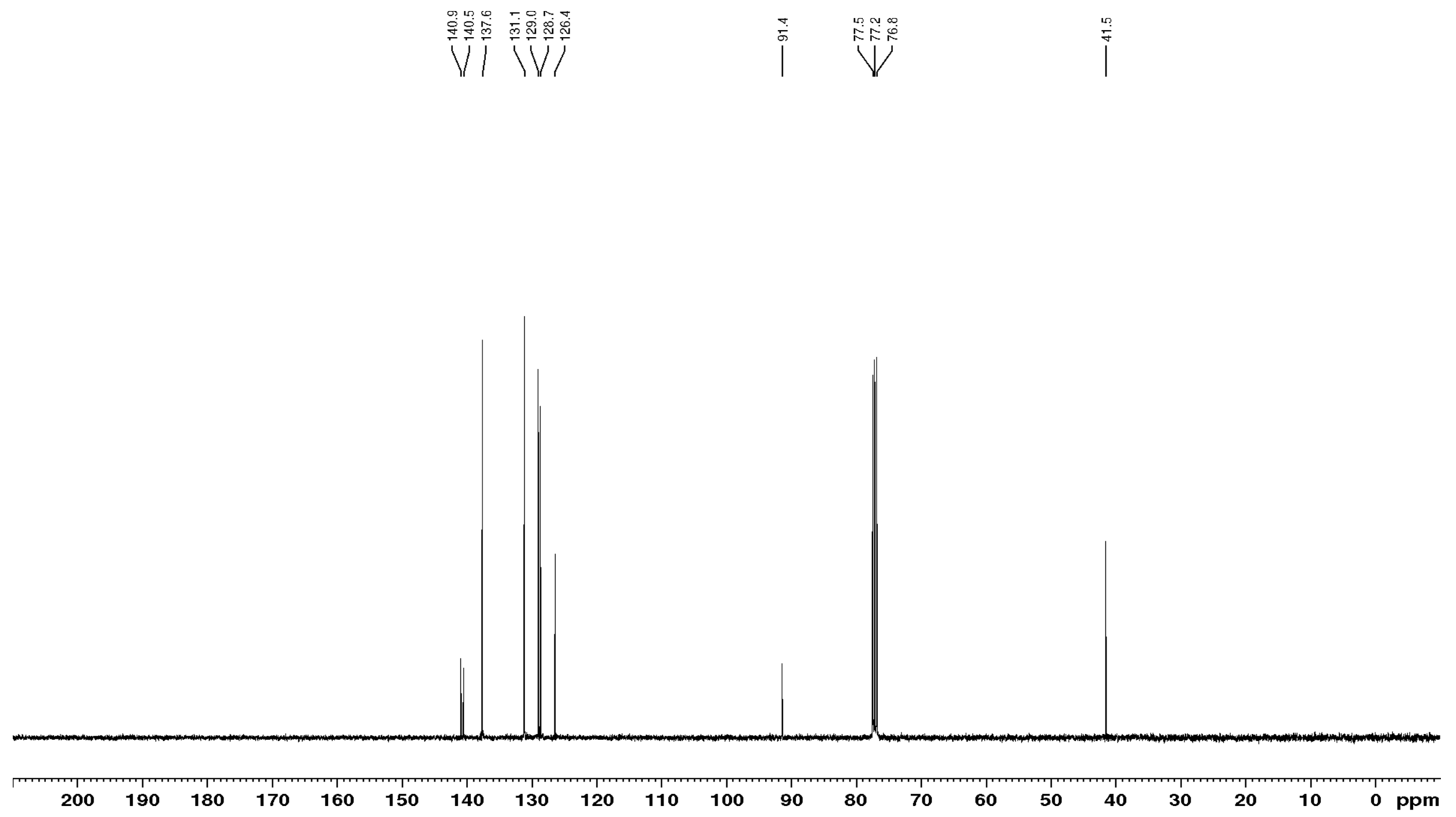
Figure S36. ${ }^{1} \mathrm{H}$ NMR spectrum ( $\left.500 \mathrm{MHz}, \mathrm{CDCl}_{3}, 298 \mathrm{~K}\right)$ of 3sa from the reaction of bromocyclopentane (1s) and benzene (2a).
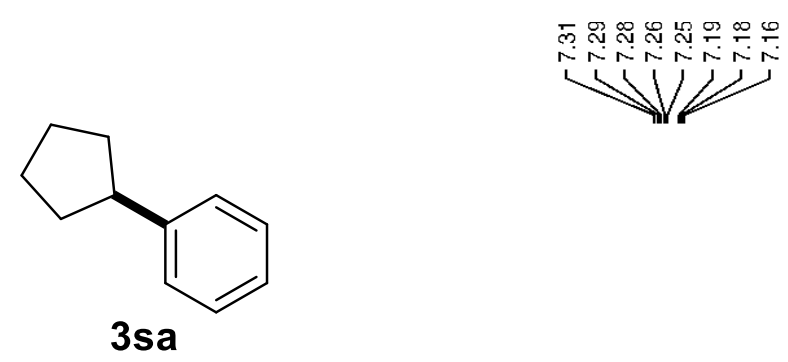

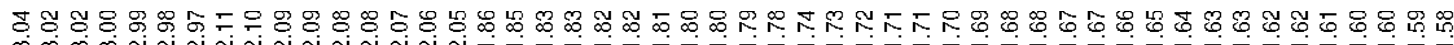

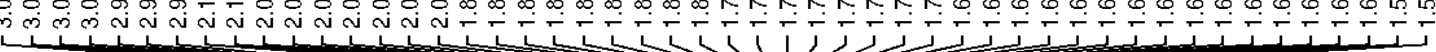

3sa




Figure S37. ${ }^{13} \mathbf{C}\left\{{ }^{1} \mathrm{H}\right\}$ NMR spectrum (126 MHz, CDCl $3,298 \mathrm{~K}$ ) of 3sa from the reaction of bromocyclopentane (1s) and benzene (2a).

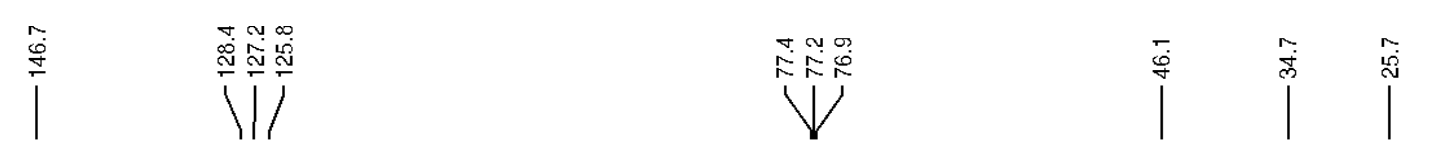

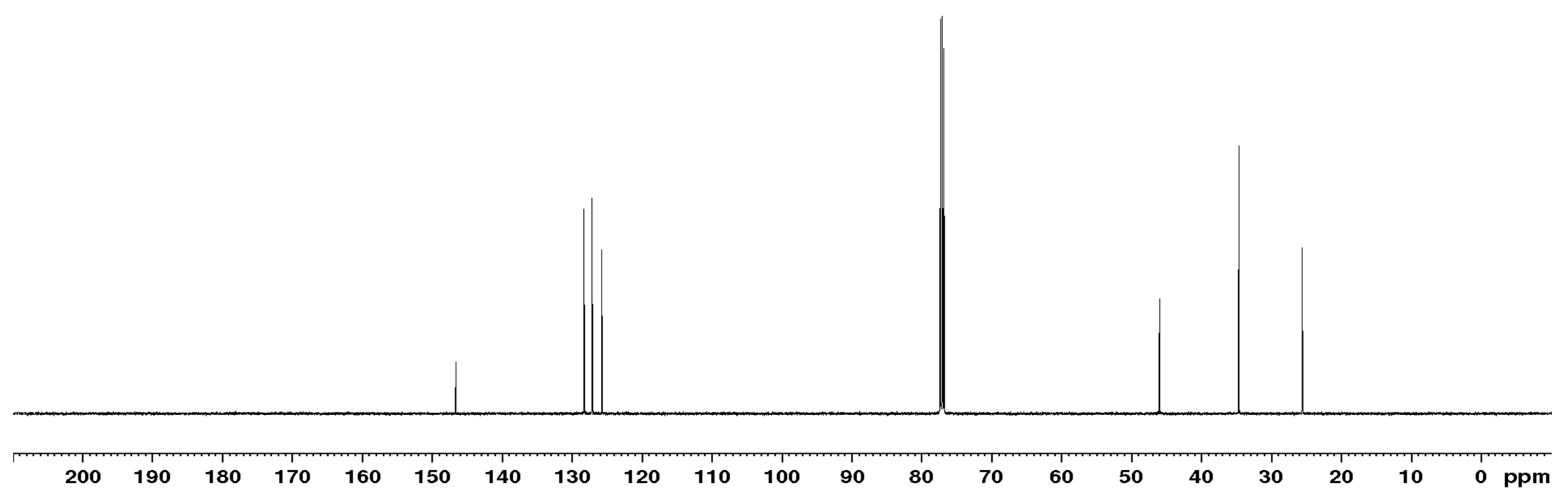


Figure S38. ${ }^{1} \mathrm{H}$ NMR spectrum $\left(500 \mathrm{MHz}, \mathrm{CDCl}_{3}, 298 \mathrm{~K}\right)$ of 3ta from the reaction of bromocyclohexane (1t) and benzene (2a).
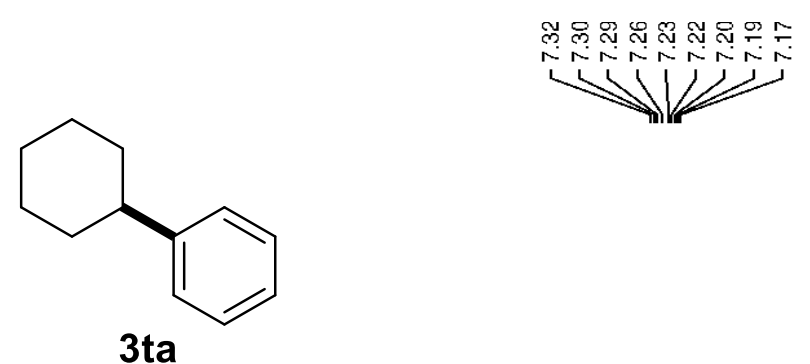

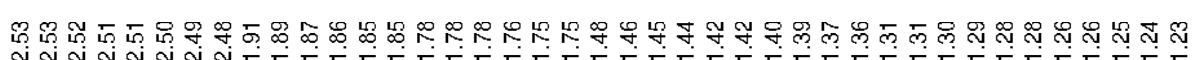

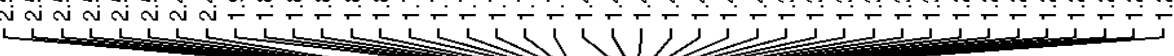

3 ta




Supporting Information for ACS Catalysis

S60

Figure S39. ${ }^{13} \mathrm{C}\left\{{ }^{1} \mathrm{H}\right\}$ NMR spectrum (126 MHz, $\left.\mathrm{CDCl}_{3}, 298 \mathrm{~K}\right)$ of 3ta from the reaction of bromocyclohexane (it) and benzene (aa).

Figure S40. ${ }^{1} \mathbf{H}$ NMR spectrum $\left(500 \mathrm{MHz}, \mathrm{CDCl}_{3}, 298 \mathrm{~K}\right)$ of 3ua from the reaction of 2-bromoadamantane (1u) and benzene (2a).


3ua




Figure S41. ${ }^{13} \mathbf{C}\left\{{ }^{1} \mathrm{H}\right\}$ NMR spectrum (126 MHz, $\left.\mathrm{CDCl}_{3}, 298 \mathrm{~K}\right)$ of 3ua from the reaction of 2-bromoadamantane (1u) and benzene (2a).
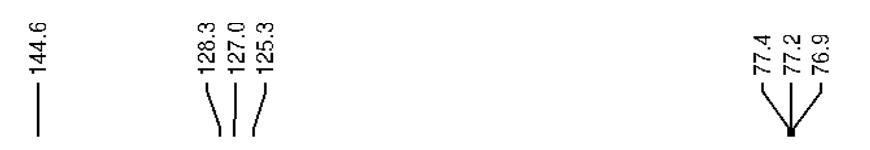

|

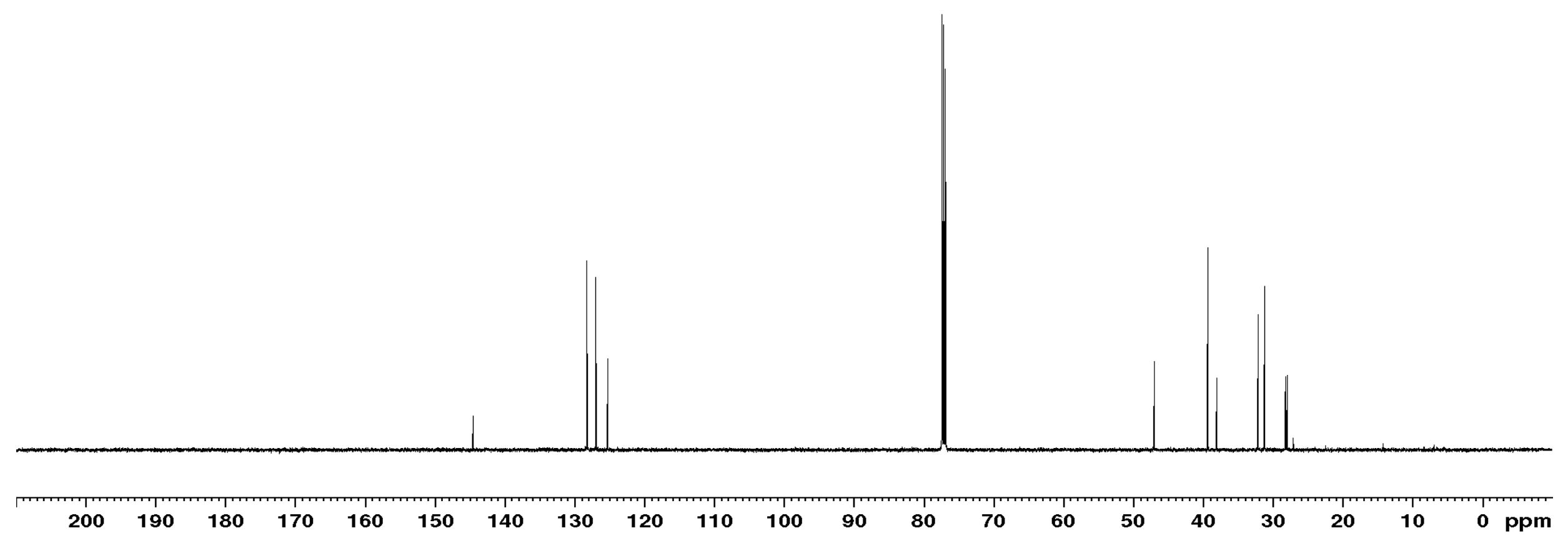




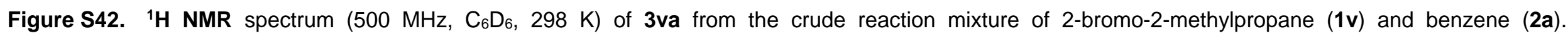
( for cyclohexane)




Figure S43. ${ }^{1} \mathrm{H}$ NMR spectrum $\left(400 \mathrm{MHz}, \mathrm{CDCl}_{3}, 298 \mathrm{~K}\right)$ of 3 wa from the reaction of 1-bromoadamantane (1w) and benzene (2a).

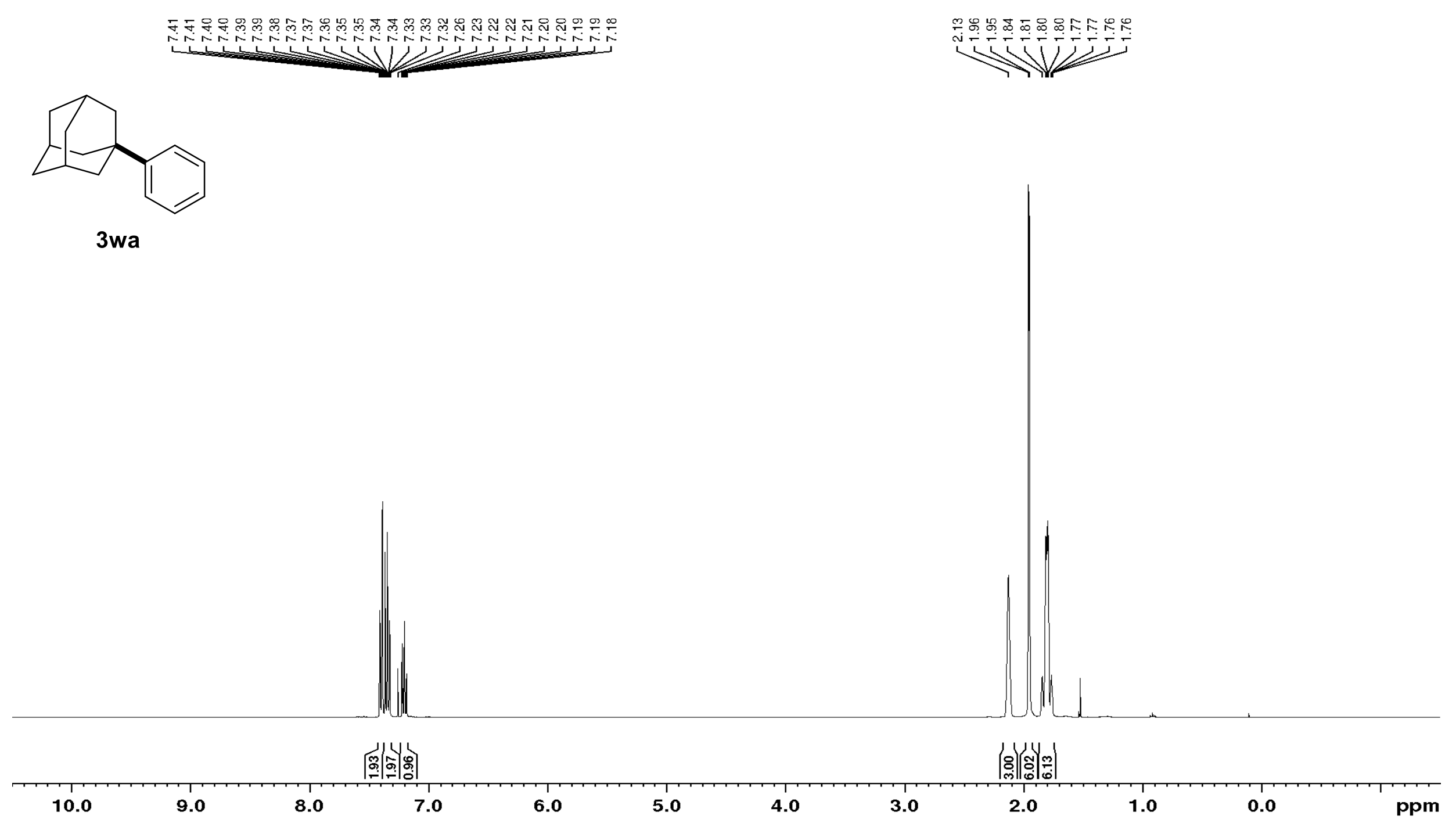


Figure S44. ${ }^{13} \mathrm{C}\left\{{ }^{1} \mathrm{H}\right\}$ NMR spectrum $\left(101 \mathrm{MHz}, \mathrm{CDCl}_{3}, 298 \mathrm{~K}\right)$ of 3 wa from the reaction of 1-bromoadamantane (1w) and benzene (2a).
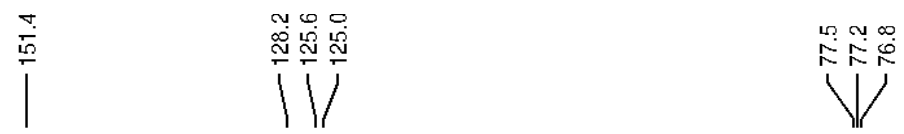

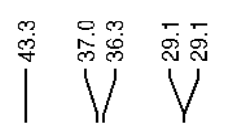

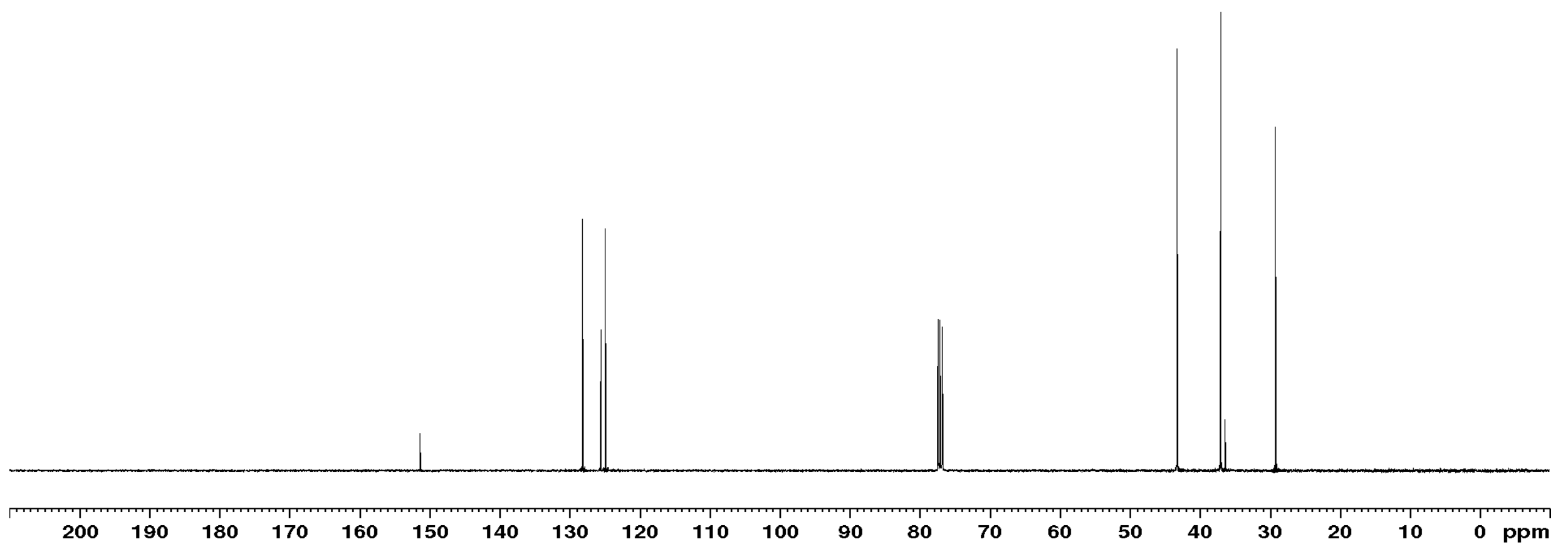


Figure S45. ${ }^{1} \mathbf{H}$ NMR spectrum $\left(500 \mathrm{MHz}, \mathrm{CDCl}_{3}, 298 \mathrm{~K}\right)$ of $3 \mathbf{c b}$ from the reaction of (3-bromopropyl)benzene (1c) and mesitylene (2b).




Figure S46. ${ }^{13} \mathrm{C}\left\{{ }^{1} \mathrm{H}\right\}$ NMR spectrum $\left(126 \mathrm{MHz}, \mathrm{CDCl}_{3}, 298 \mathrm{~K}\right)$ of $3 \mathbf{c b}$ from the reaction of (3-bromopropyl)benzene (1c) and mesitylene (2b).
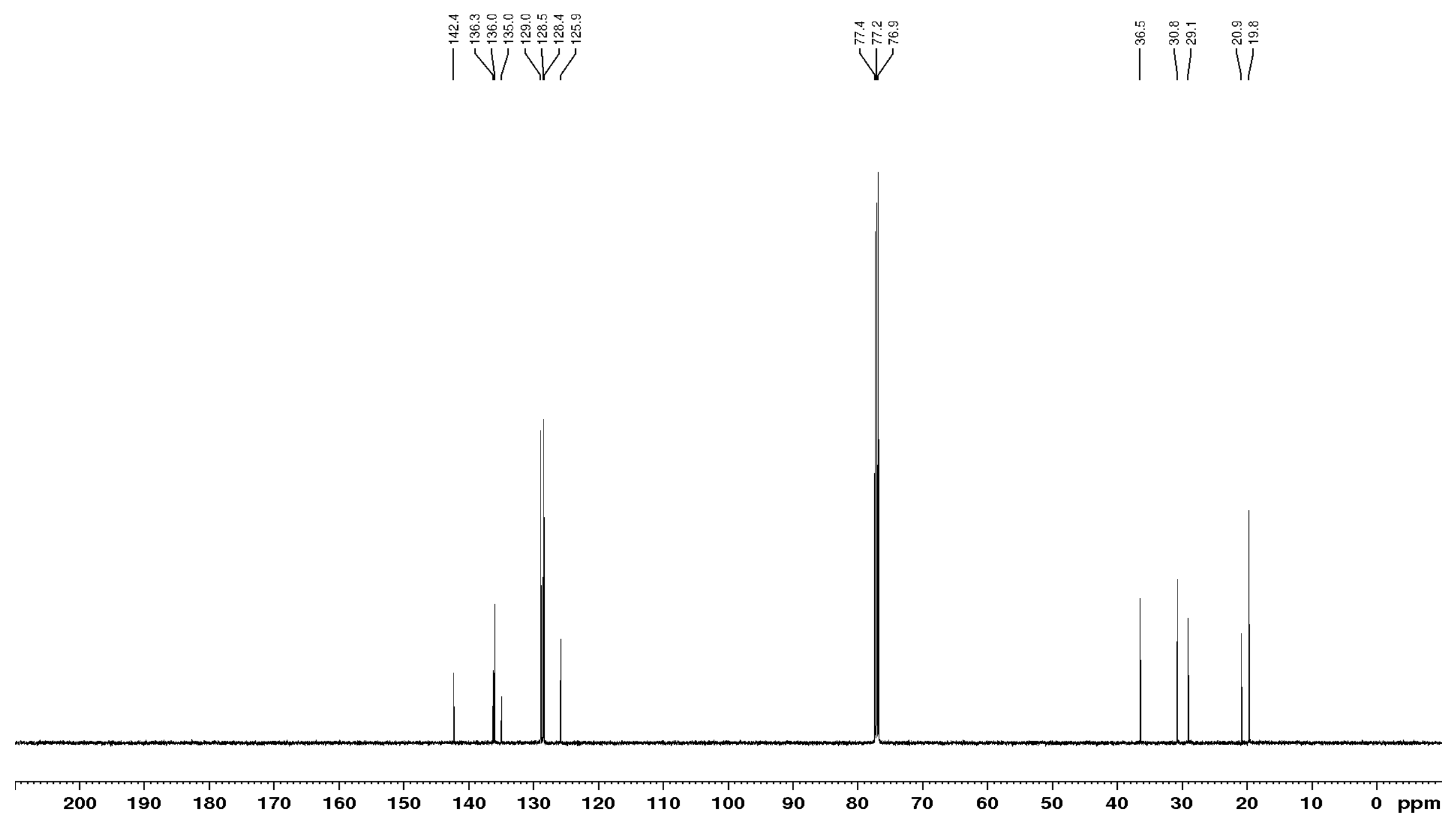
Figure S47. ${ }^{1} \mathrm{H}$ NMR spectrum ( $\left.500 \mathrm{MHz}, \mathrm{CDCl}_{3}, 298 \mathrm{~K}\right)$ of $3 \mathrm{eb}$ from the reaction of benzyl bromide (1e) and mesitylene (2b).

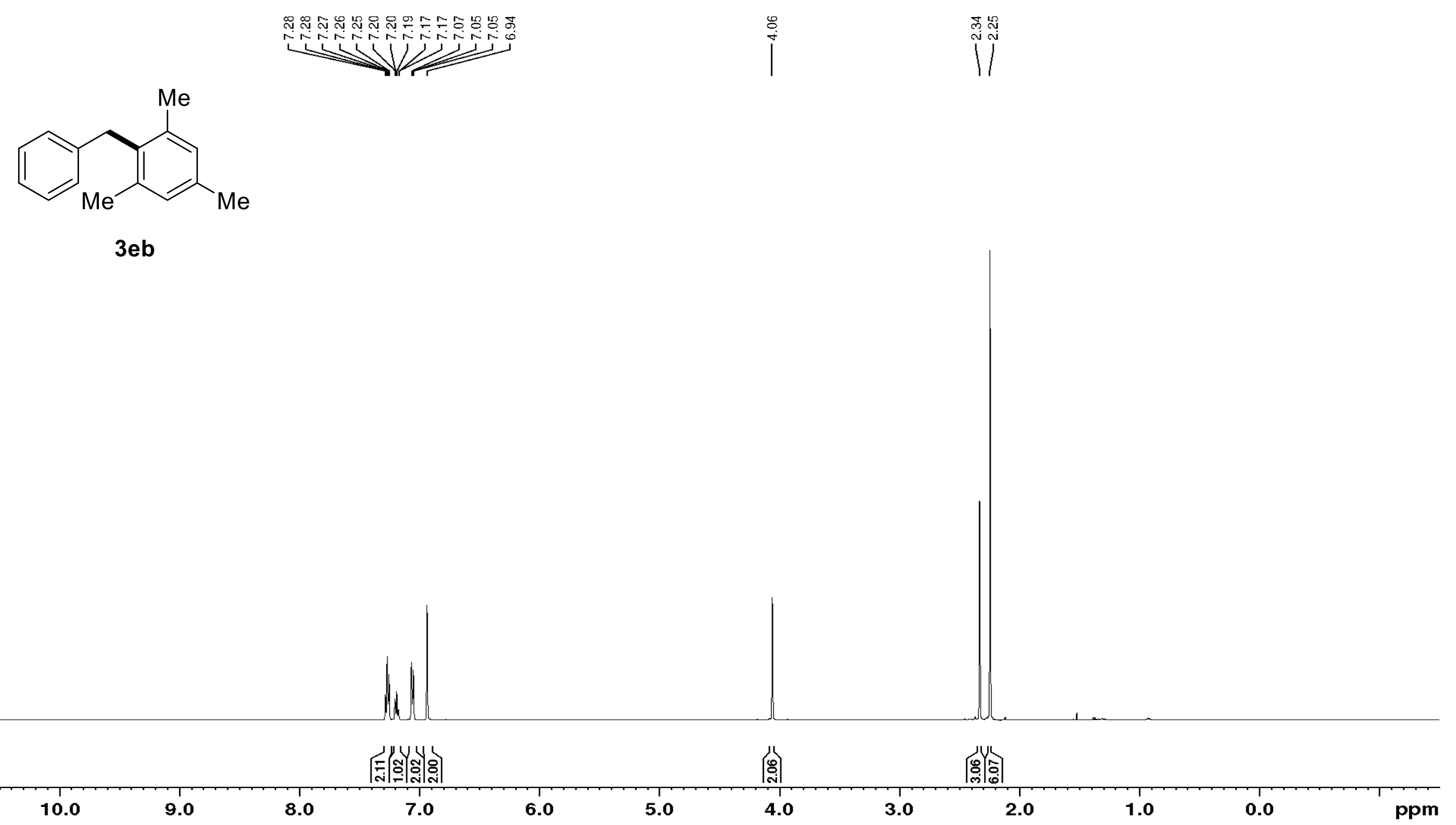


Figure S48. ${ }^{13} \mathrm{C}\left\{{ }^{1} \mathrm{H}\right\}$ NMR spectrum $\left(126 \mathrm{MHz}, \mathrm{CDCl}_{3}, 298 \mathrm{~K}\right)$ of $3 \mathbf{e b}$ from the reaction of benzyl bromide (1e) and mesitylene (2b).

Figure S49. $\quad{ }^{1} \mathbf{H}$ NMR spectrum (500 MHz, $\left.\mathrm{CDCl}_{3}, 298 \mathrm{~K}\right)$ of $3 \mathbf{e c}$ from the reaction of benzyl bromide (1e) and $p$-xylene (2c). $\left({ }^{*}\right.$ for diphenylmethane)
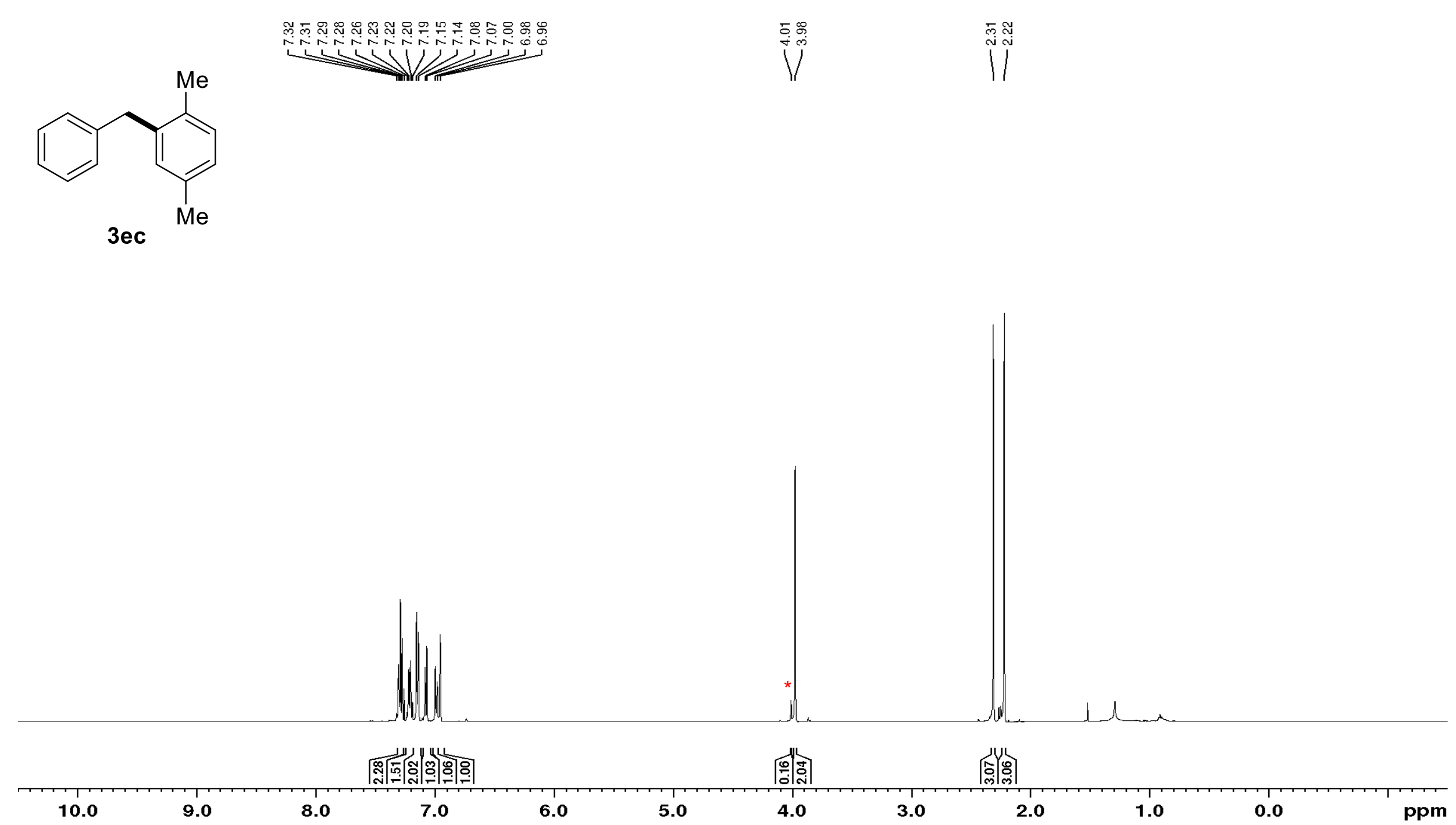
Figure S50. ${ }^{13} \mathrm{C}\left\{{ }^{1} \mathrm{H}\right\}$ NMR spectrum $\left(126 \mathrm{MHz}, \mathrm{CDCl}_{3}, 298 \mathrm{~K}\right)$ of $3 \mathbf{e c}$ from the reaction of benzyl bromide (1e) and $p$-xylene (2c).

Figure S51. ${ }^{1} \mathbf{H}$ NMR spectrum $\left(500 \mathrm{MHz}, \mathrm{CDCl}_{3}, 298 \mathrm{~K}\right)$ of the reaction of benzyl bromide (1e) and toluene (2d) $\left({ }^{*}\right.$ for $\mathbf{3 e d}$; $v$ for $\mathbf{3 e d}$; \# for $\mathbf{3 e d " )}$



b) Pure 3fa (in $\mathrm{CDCl}_{3}$ )

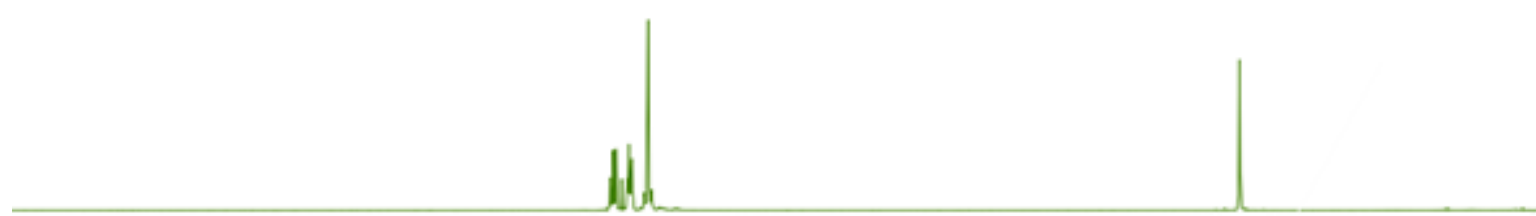

c) Pure 3ga (in $\mathrm{CDCl}_{3}$ )

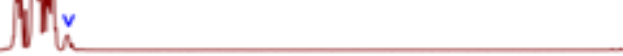

3ed (4-Me) + 3ed' (3-Me) + 3ed" (2-Me)

d) Pure 3ha (in $\mathrm{CDCl}_{3}$ )



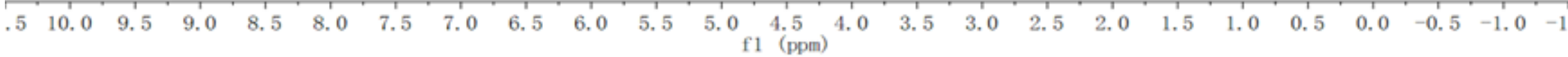


Figure S52. ${ }^{1} \mathbf{H}$ NMR spectrum $\left(500 \mathrm{MHz}, \mathrm{CDCl}_{3}, 298 \mathrm{~K}\right)$ of the reaction of benzyl bromide (1e) and naphthalene (2d). ( ${ }^{*}$ for 3ee and v for 3ee')

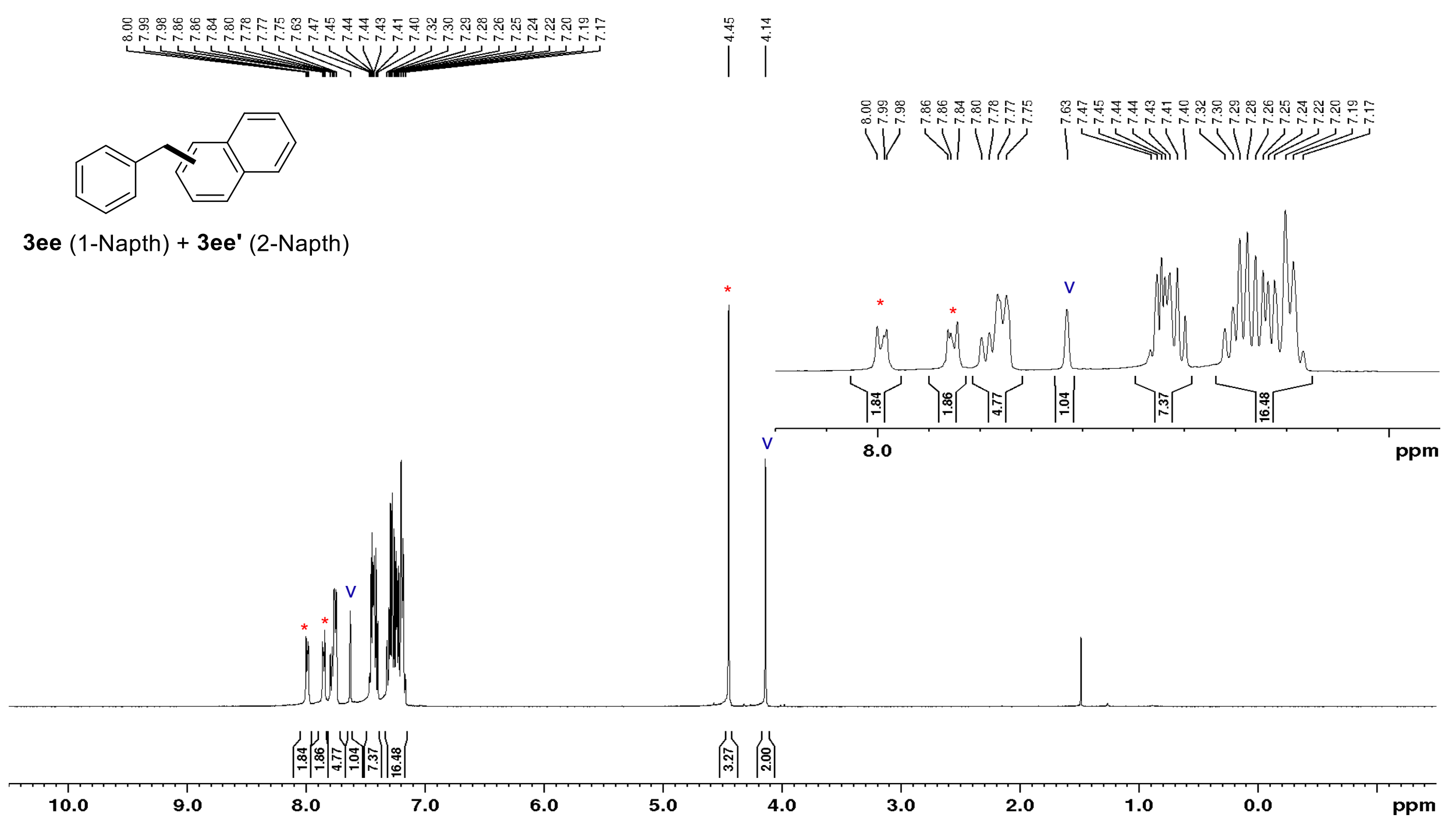


Figure S53. ${ }^{1} \mathbf{H}$ NMR spectrum $\left(500 \mathrm{MHz}, \mathrm{C}_{6} \mathrm{D}_{6}, 298 \mathrm{~K}\right)$ of crude $\mathbf{3} \mathbf{x a}$ from the reaction of bromoethane (1x) and benzene (2a). ( ${ }^{*}$ for ethylbenzene $\mathbf{3} \mathbf{x a}$; $\mathbf{v}$ for cyclohexane)
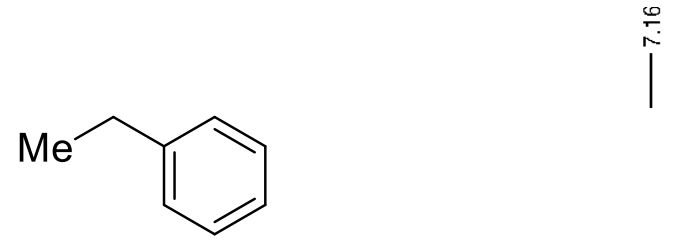

$3 \times a$

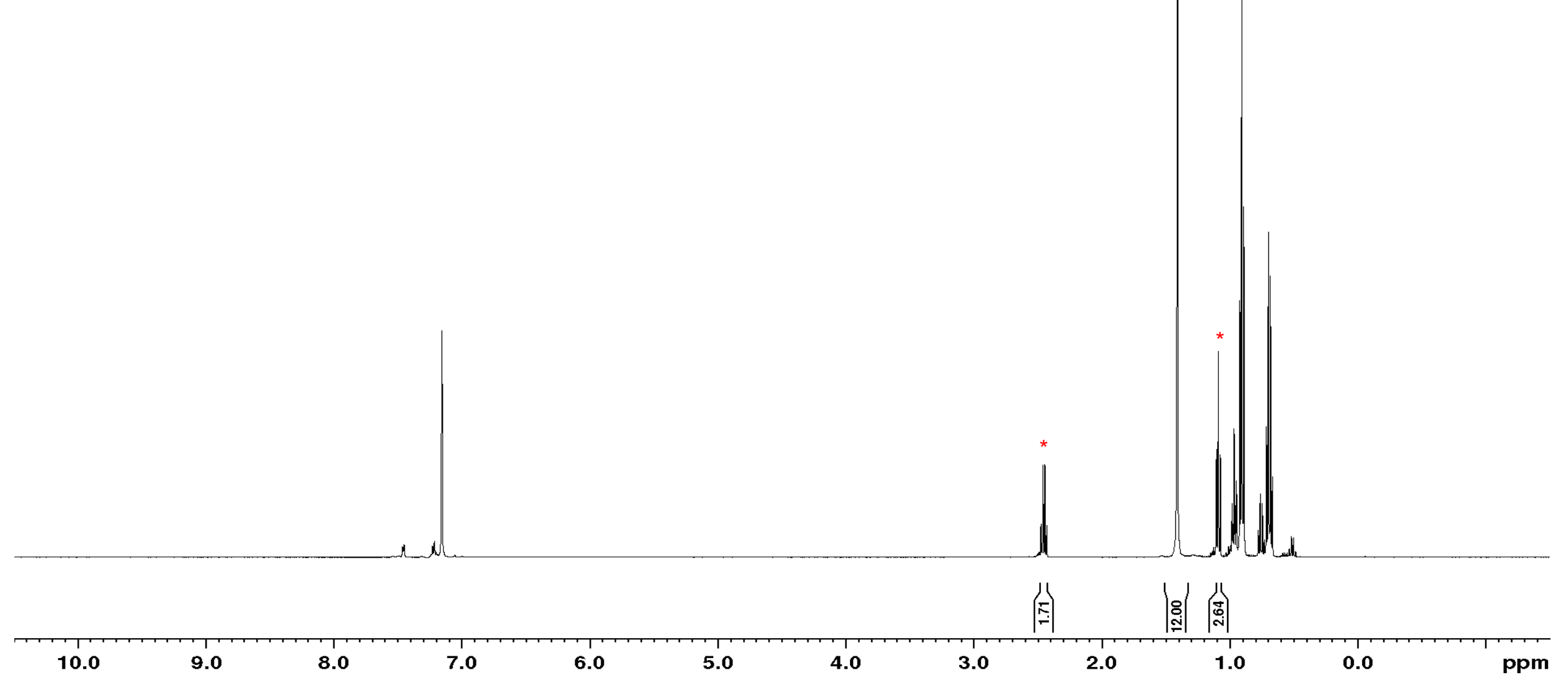


Figure S54. ${ }^{1} \mathrm{H}$ NMR spectrum $\left(400 \mathrm{MHz}, \mathrm{C}_{6} \mathrm{D}_{6}, 298 \mathrm{~K}\right)$ of crude 10 from the reaction of (4-bromobutyl)benzene (1y). ( ${ }^{*}$ for 1,2,3,4-tetrahydronaphthalene 10; $\mathbf{v}$ for cyclohexane)


10

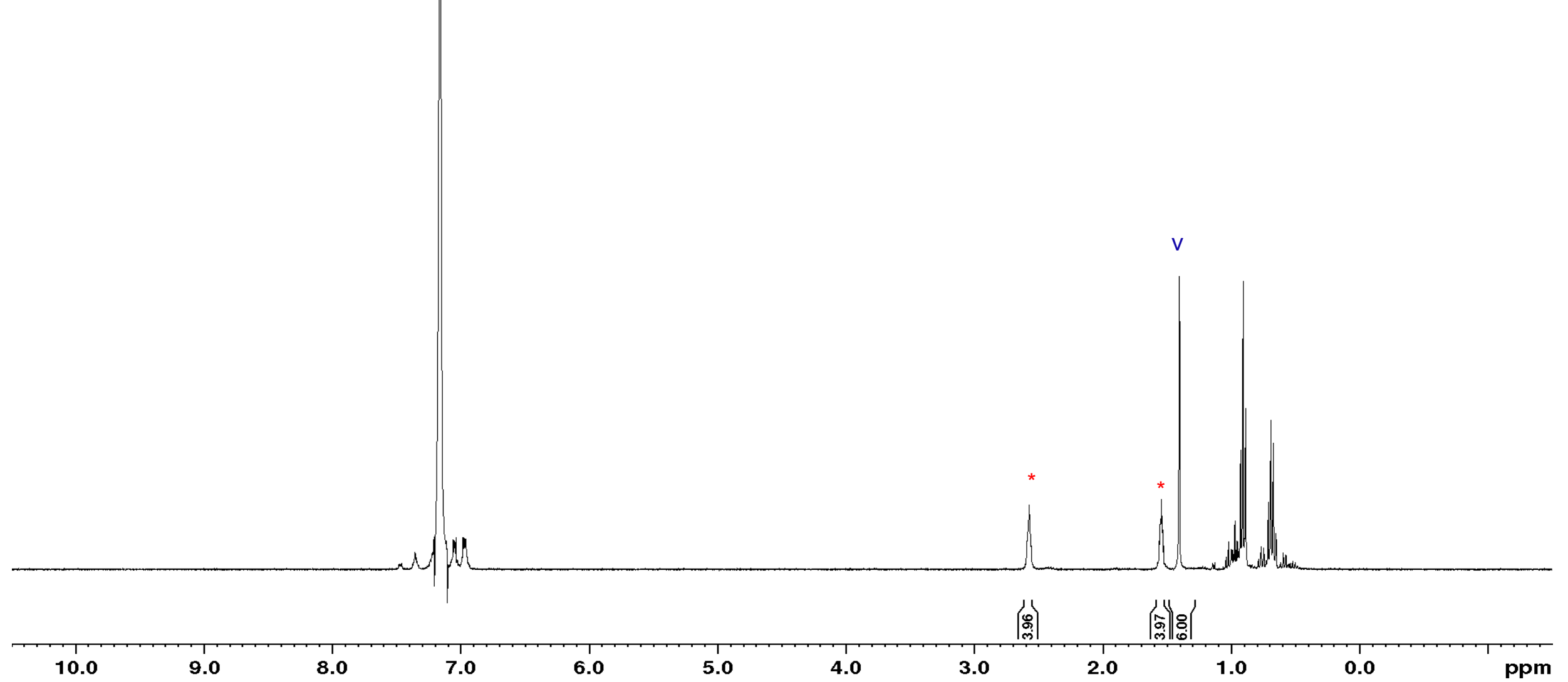




\section{References}

[S1] Reed, C. A. $\mathrm{H}^{+}, \mathrm{CH}_{3}{ }^{+}$, and $\mathrm{R}_{3} \mathrm{Si}^{+}$Carborane Reagents: When Triflates Fail. Acc. Chem. Res. 2010, 43, 121-128.

[S2] Wu, Q.; Qu, Z. W.; Omann, L.; Irran, E.; Klare, H. F. T.; Oestreich, M. Cleavage of Unactivated $\mathrm{Si}-\mathrm{C}\left(\mathrm{sp}^{3}\right)$ Bonds with Reed's Carborane Acids: Formation of Known and Unknown Silylium lons. Angew. Chem., Int. Ed. 2018, 57, 9176-9179.

[S3] Xie, Z. W.; Bau, R.; Benesi, A.; Reed, C. A. The Silylium Ion ( $\left.\mathrm{R}_{3} \mathrm{Si}^{+}\right)$Problem: Effect of Alkyl Substituents R. Organometallics 1995, 14, 3933-3941.

[S4] Omann, L.; Pudasaini, B.; Irran, E.; Klare, H. F. T.; Baik, M. H.; Oestreich, M. Thermodynamic versus kinetic control in substituent redistribution reactions of silylium ions steered by the counteranion. Chem. Sci. 2018, 9, 5600-5607.

[S5] He, T.; Wang, G. Q.; Long, P. W.; Kemper, S.; Irran, E.; Klare, H. F. T.; Oestreich, M. Intramolecular Friedel-Crafts alkylation with a silylium-ion-activated cyclopropyl group: formation of tricyclic ring systems from benzyl-substituted vinylcyclopropanes and hydrosilanes. Chem. Sci. 2021, 12, 569-575.

[S6] Wu, Q.; Roy, A.; Irran, E.; Qu, Z. W.; Grimme, S.; Klare, H. F. T.; Oestreich, M. Catalytic Difunctionalization of Unactivated Alkenes with Unreactive Hexamethyldisilane through Regeneration of Silylium lons. Angew. Chem., Int. Ed. 2019, 58, 17307-17311.

[S7] Harris, R. K.; Becker, E. D.; De Menezes, S. M. C.; Goodfellow, R.; Granger, P. NMR nomenclature. Nuclear spin properties and conventions for chemical shifts (IUPAC recommendations 2001). Pure Appl. Chem. 2001, 73, 1795-1818.

[S8] Mita, T.; Tanaka, H.; Michigami, K.; Sato, Y. Ruthenium-Catalyzed C-H Silylation of 1Arylpyrazole Derivatives and Fluoride-Mediated Carboxylation: Use of Two Nitrogen Atoms of the Pyrazole Group. Synlett. 2014, 25, 1291-1294.

[S9] Liu, X. W.; Zarate, C.; Martin, R. Base-Mediated Defluorosilylation of $\mathrm{C}\left(\mathrm{sp}^{2}\right)-\mathrm{F}$ and C(sp $\left.{ }^{3}\right)-F$ Bonds. Angew. Chem., Int. Ed. 2019, 58, 2064-2068.

[S10] Yamanoi, Y.; Nishihara, H. Direct and Selective Arylation of Tertiary Silanes with Rhodium Catalyst. J. Org. Chem. 2008, 73, 6671-6678.

[S11] Yamanoi, Y. Palladium-Catalyzed Silylations of Hydrosilanes with Aryl Halides Using Bulky Alkyl Phosphine. J. Org. Chem. 2005, 70, 9607-9609.

[S12] Jang, J.; Byun, S.; Kim, B. M.; Lee, S. Arylsilylation of aryl halides using the magnetically recyclable bimetallic $\mathrm{Pd}-\mathrm{Pt}-\mathrm{Fe}_{3} \mathrm{O}_{4}$ catalyst. Chem. Commun. 2018, 54, 3492-3495.

[S13] Gupta, L.; Hoepker, A. C.; Singh, K. J.; Collum, D. B. Lithium Diisopropylamide-Mediated Ortholithiations: Lithium Chloride Catalysis. J. Org. Chem. 2009, 74, 2231-2233.

[S14] Heiss, C.; Marzi, E.; Schlosser, M. Buttressing effects rerouting the deprotonation and functionalization of 1,3-dichloro- and 1,3-dibromobenzene. Eur. J. Org. Chem. 2003, 46254629. 
[S15] Dryzhakov, M.; Moran, J. Autocatalytic Friedel-Crafts Reactions of Tertiary Aliphatic Fluorides Initiated by $\mathrm{B}\left(\mathrm{C}_{6} \mathrm{~F}_{5}\right)_{3} \cdot \mathrm{H}_{2} \mathrm{O}$. ACS Catal. 2016, 6, 3670-3673.

[S16] Yang, C. T.; Zhang, Z. Q.; Liu, Y. C.; Liu, L. Copper-Catalyzed Cross-Coupling Reaction of Organoboron Compounds with Primary Alkyl Halides and Pseudohalides. Angew. Chem., Int. Ed. 2011, 50, 3904-3907.

[S17] Gieshoff, T. N.; Chakraborty, U.; Villa, M.; Jacobi von Wangelin, A. Alkene Hydrogenations by Soluble Iron Nanocluster Catalysts. Angew. Chem., Int. Ed. 2017, 56, 3585-3589.

[S18] Czyz, M. L.; Taylor, M. S.; Horngren, T. H.; Polyzos, A. Reductive Activation and Hydrofunctionalization of Olefins by Multiphoton Tandem Photoredox Catalysis. ACS Catal. 2021, 11, 5472-5480.

[S19] Maity, P.; Shacklady-McAtee, D. M.; Yap, G. P. A.; Sirianni, E. R.; Watson, M. P. NickelCatalyzed Cross Couplings of Benzylic Ammonium Salts and Boronic Acids: Stereospecific Formation of Diarylethanes via C-N Bond Activation. J. Am. Chem. Soc. 2013, 135, $280-$ 285.

[S20] Lv, L. Y.; Zhu, D. H.; Tang, J. T.; Qiu, Z. H.; Li, C. C.; Gao, J.; Li, C. J. Cross-Coupling of Phenol Derivatives with Umpolung Aldehydes Catalyzed by Nickel. ACS Catal. 2018, 8, 4622-4627.

[S21] Nozawa-Kumada, K.; Ito, S.; Noguchi, K.; Shigeno, M.; Kondo, Y. Super electron donormediated reductive desulfurization reactions. Chem. Commun. 2019, 55, 12968-12971.

[S22] Kuwano, R.; Yokogi, M. Suzuki-Miyaura Cross-Coupling of Benzylic Carbonates with Arylboronic Acids. Org. Lett. 2005, 7, 945-947.

[S23] Cheng, Y.; Dong, W. R.; Wang, L.; Parthasarathy, K.; Bolm, C. Iron-Catalyzed HeteroCross-Dehydrogenative Coupling Reactions of Sulfoximines with Diarylmethanes: A New Route to N-Alkylated Sulfoximines. Org. Lett. 2014, 16, 2000-2002.

[S24] St. Denis, J. D.; Scully, C. C. G.; Lee, C. F.; Yudin, A. K. Development of the Direct SuzukiMiyaura Cross-Coupling of Primary B-Alkyl MIDA-boronates and Aryl Bromides. Org. Lett. 2014, 16, 1338-1341.

[S25] Baguley, T. D.; Xu, H. C.; Chatterjee, M.; Nairn, A. C.; Lombroso, P. J.; Ellman, J. A. Substrate-Based Fragment Identification for the Development of Selective, Nonpeptidic Inhibitors of Striatal-Enriched Protein Tyrosine Phosphatase. J. Med. Chem. 2013, 56, 7636-7650.

[S26] Vasilopoulos, A.; Zultanski, S. L.; Stahl, S. S. Feedstocks to Pharmacophores: CuCatalyzed Oxidative Arylation of Inexpensive Alkylarenes Enabling Direct Access to Diarylalkanes. J. Am. Chem. Soc. 2017, 139, 7705-7708.

[S27] Ghorai, S. K.; Jin, M.; Hatakeyama, T.; Nakamura, M. Cross-Coupling of Non-activated Chloroalkanes with Aryl Grignard Reagents in the Presence of Iron/N-Heterocyclic Carbene Catalysts. Org. Lett. 2012, 14, 1066-1069.

[S28] Powell, D. A.; Maki, T.; Fu, G. C. Stille Cross-Couplings of Unactivated Secondary Alkyl Halides Using Monoorganotin Reagents. J. Am. Chem. Soc. 2005, 127, 510-511. 
[S29] Fout, A. R.; Bailey, B. C.; Buck, D. M.; Tan, H. J.; Huffman, J. C.; Baik, M. H.; Mindiola, D. J., Synthetic and Mechanistic Studies of the Ring Opening and Denitrogenation of Pyridine and Picolines by Ti-C Multiple Bonds. Organometallics 2010, 29, 5409-5422.

[S30] Toriyama, F.; Cornella, J.; Wimmer, L.; Chen, T. G.; Dixon, D. D.; Creech, G.; Baran, P. S., Redox-Active Esters in Fe-Catalyzed C-C Coupling. J. Am. Chem. Soc. 2016, 138, 1113211135.

[S31] Fu, L. Y.; Chen, Q.; Wang, Z. H.; Nishihara, Y., Palladium-Catalyzed Decarbonylative Alkylation of Acyl Fluorides. Org. Lett. 2020, 22, 2350-2353.

[S32] Bering, L.; Jeyakumar, K.; Antonchick, A. P., Metal-Free C-O Bond Functionalization: Catalytic Intramolecular and Intermolecular Benzylation of Arenes. Org. Lett. 2018, 20 , 3911-3914.

[S33] Tang, R. J.; Milcent, T.; Crousse, B., Bisulfate Salt-Catalyzed Friedel-Crafts Benzylation of Arenes with Benzylic Alcohols. J. Org. Chem. 2018, 83, 14001-14009.

[S34] Suga, T.; Ukaji, Y., Nickel-Catalyzed Cross-Electrophile Coupling between Benzyl Alcohols and Aryl Halides Assisted by Titanium Co-reductant. Org. Lett. 2018, 20, 7846-7850.

[S35] Zhang, J.; Lu, G. S.; Xu, J.; Sun, H. M.; Shen, Q., Nickel-Catalyzed Reductive CrossCoupling of Benzyl Chlorides with Aryl Chlorides/Fluorides: A One-Pot Synthesis of Diarylmethanes. Org. Lett. 2016, 18, 2860-2863.

[S36] Sakai, N.; Nakajima, T.; Yoneda, S.; Konakahara, T.; Ogiwara, Y., Gallium-Catalyzed Reductive Chlorination of Carboxylic Acids with Copper(II) Chloride. J. Org. Chem. 2014, 79, 10619-10623. 